\title{
THE ROLE OF THE ASTROCYTIC AND MICROGLIAL
} ARYL HYDROCARBON RECEPTOR IN CNS DEMYELINATION

\section{Doctoral Thesis}

\author{
In partial fulfilment of the requirements for the degree \\ “Doctor rerum naturalium (Dr. rer. nat.)" \\ in the Molecular Medicine Study Program \\ at the Georg-August University Göttingen
}

submitted by

\section{Susanne Schmid}

born in Dachau, Germany 


\section{MEMBERS OF THE THESIS COMMITTEE}

\section{Supervisor (Reviewer)}

Prof. Dr. Wolfgang Brück

Chair of the Executive Board, Dean of the Medical Faculty

University Medical Center, Georg-August University Göttingen

\section{Second member of the thesis committee (Reviewer)}

Prof. Dr. Alexander Flügel

Institute of Neuroimmunology and Multiple Sclerosis Research

University Medical Center, Georg-August University Göttingen

\section{Third member of the thesis committee}

Prof. Dr. Peter Burfeind

Department of Human Genetics

University Medical Center, Georg-August University Göttingen

\section{MEMBERS OF THE EXAMINATION BOARD}

Prof. Dr. Dr. Hannelore Ehrenreich

Department of Clinical Neuroscience

Max Planck Institute of Experimental Medicine, Göttingen

Prof. Dr. Heidi Hahn

Department of Human Genetics, Section of Developmental Genetics

University Medical Center, Georg-August University Göttingen

Prof. Dr. Frauke Alves

Department of Molecular Biology of Neuronal Signals

Max Planck Institute of Experimental Medicine, Göttingen 


\section{AfFIDAVIT}

Here I declare that my doctoral thesis entitled "The role of the astrocytic and microglial aryl hydrocarbon receptor in CNS demyelination" has been written independently with no other sources and aids than quoted.

\section{Susanne Schmid}

Göttingen, August 2020 
Meinem lieben Manuel 


\section{LIST OF PUBLICATIONS}

\section{Original articles}

Kramann N, Menken L, Pförtner R, Schmid SN, Stadelmann C, Wegner C, Brück W. (2019). Glial fibrillary acidic protein expression alters astrocytic chemokine release and protects mice from cuprizone-induced demyelination. Glia. 67(7):1308-1319.

Merk DJ, Ohli J, Merk ND, Thatikonda V, Morrissy S, Schoof M, Schmid SN, Harrison L, Filser S, Ahlfeld J, Erkek S, Raithatha K, Andreska T, Weißhaar M, Launspach M, Neumann JE, Shakarami M, Plenker D, Marra MA, Li Y, Mungall AJ, Moore RA, Ma Y, Jones SJM, Lutz B, ErtlWagner B, Rossi A, Wagener R, Siebert R, Jung A, Eberhart CG, Lach B, Sendtner M, Pfister SM, Taylor MD, Chavez L, Kool M, Schüller U. (2018). Opposing Effects of CREBBP Mutations Govern the Phenotype of Rubinstein-Taybi Syndrome and Adult SHH Medulloblastoma. Dev Cell. 44:709-724.

\section{Abstract}

Schmid SN, Brück W, Kramann N

The role of the aryl hydrocarbon receptor in glial cells during demyelination $34^{\text {th }}$ Congress of the European Committee for Treatment and Research in Multiple Sclerosis, October $10^{\text {th }}-12^{\text {th }} 2018$, Berlin, Germany, Poster session 


\section{TABLE OF CONTENTS}

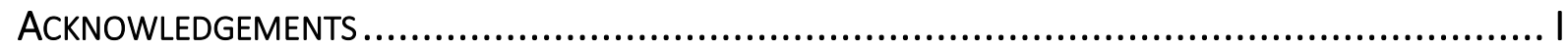

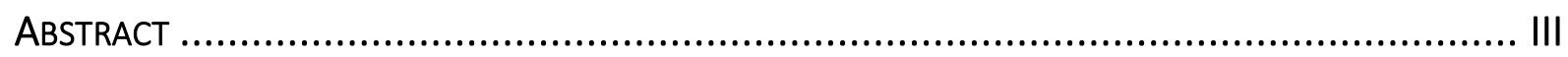

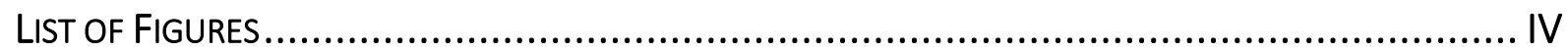

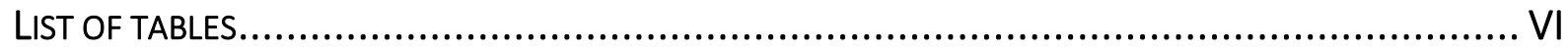

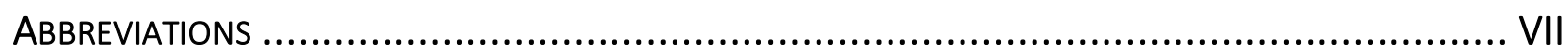

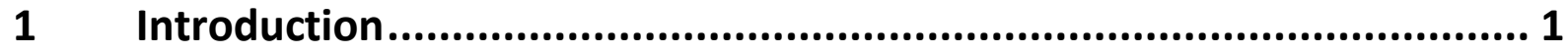

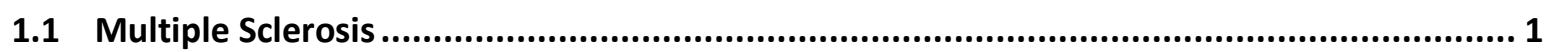

1.1.1 Symptoms, diagnosis and clinical course .......................................................................... 1

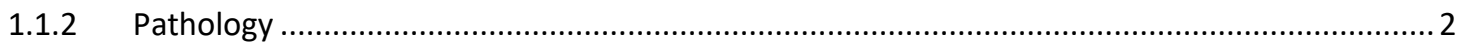

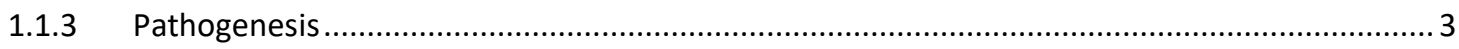

1.1.4 The role of astrocytes and microglia in CNS health and disease ......................................... 5

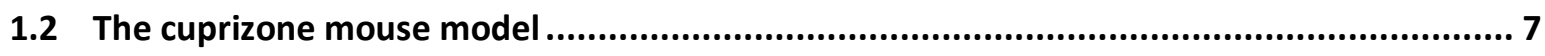

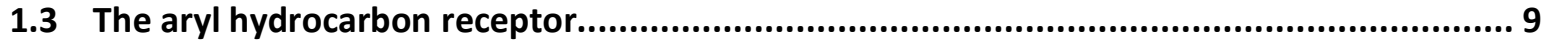

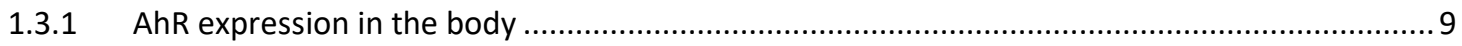

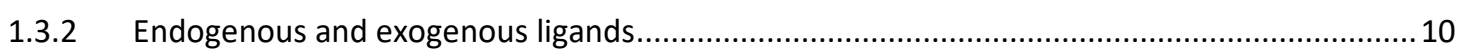

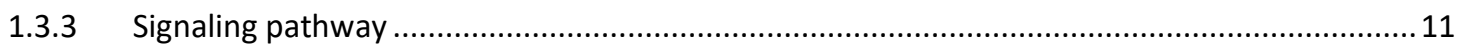

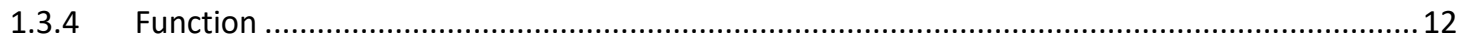

1.3.5 Constitutive AhR knockout animals ........................................................................... 12

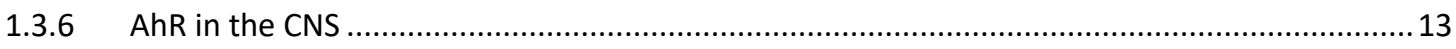

1.4 Laquinimod (LAQ) - a novel immunomodulator ................................................... 14

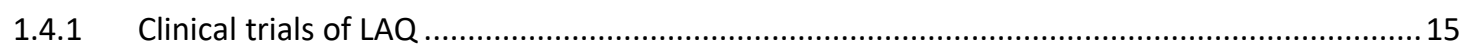

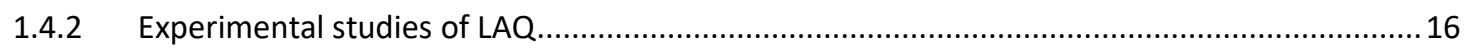

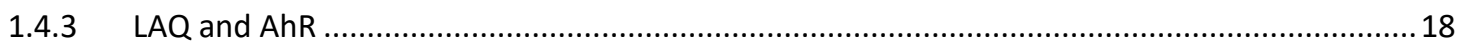

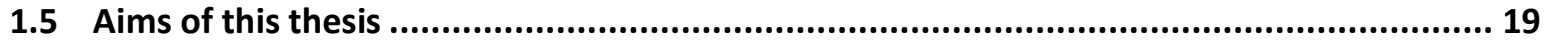

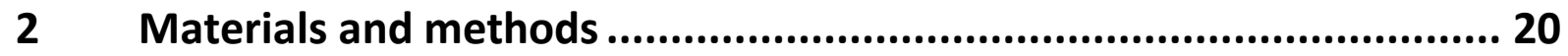

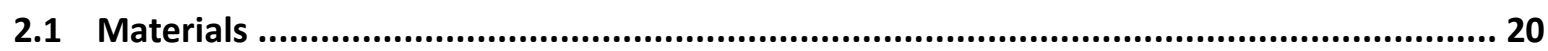

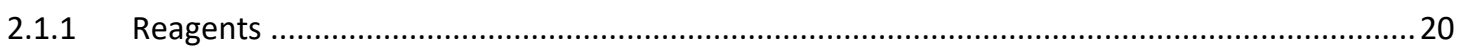




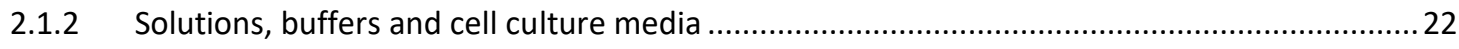

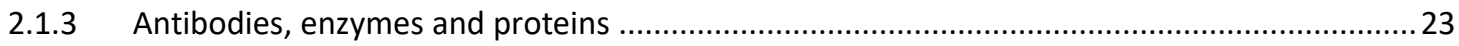

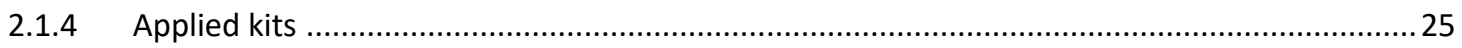

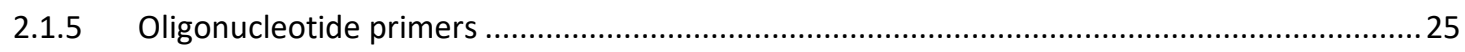

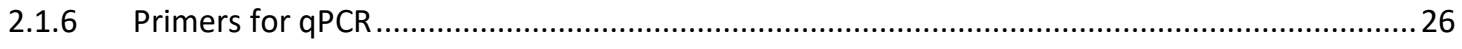

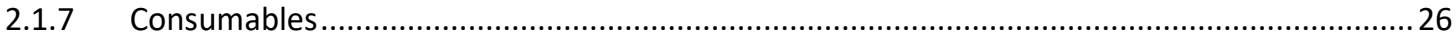

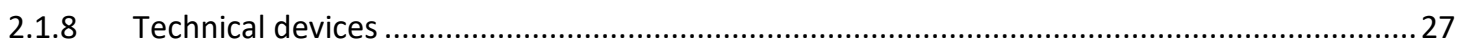

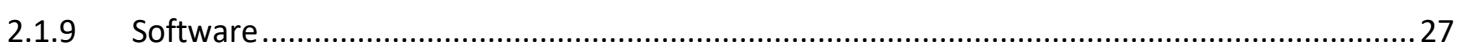

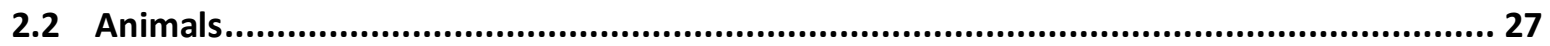

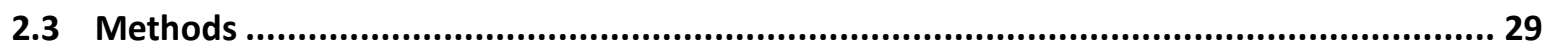

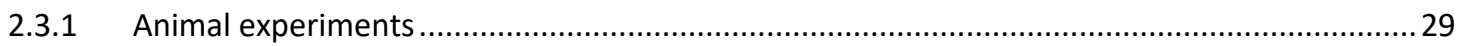

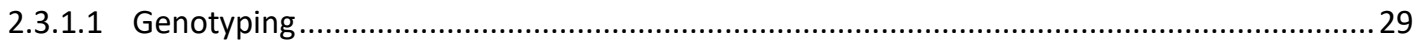

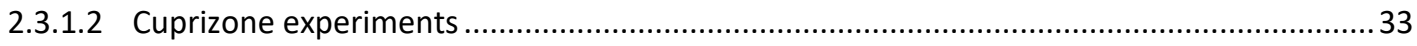

2.3.1.2.1 Tamoxifen preparation and treatment ….......................................................... 34

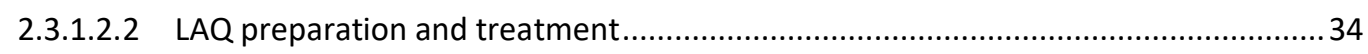

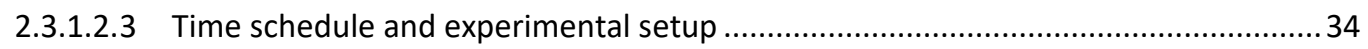

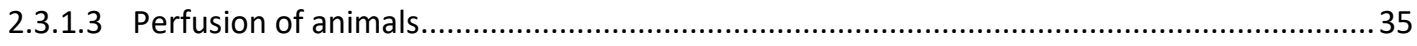

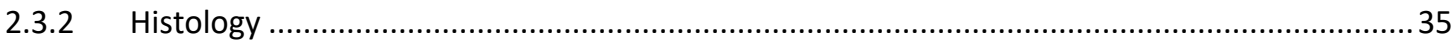

2.3.2.1 Cutting, deparaffinization and hydration of paraffin-embedded sections..........................35

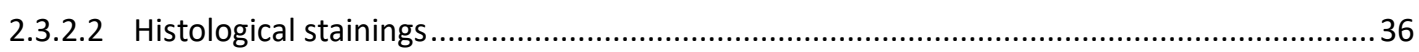

2.3.2.2.1 Luxol Fast Blue - Periodic Acid Schiff (LFB-PAS) staining ......................................... 36

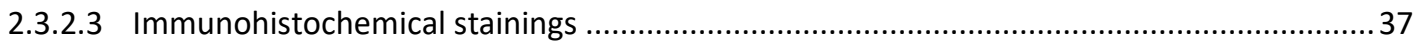

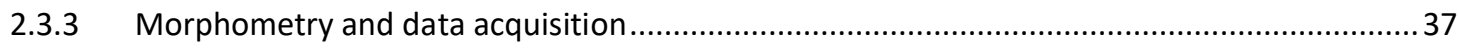

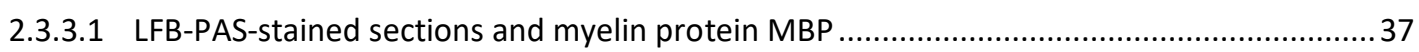

2.3.3.2 Mac3-positive microglia, APP-positive spheroids and p25-positive oligodendrocytes.........38

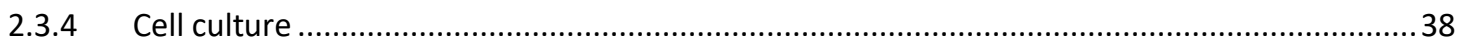

2.3.4.1 Isolation of astrocytes and microglia from newborn mice ............................................38

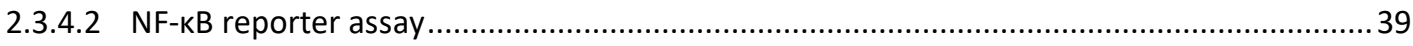

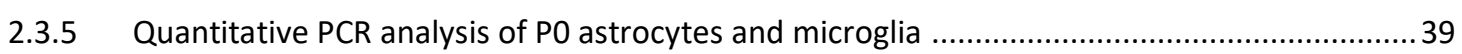

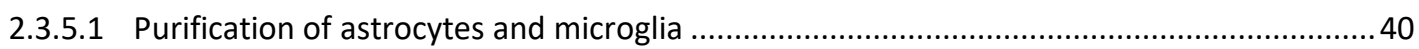

2.3.5.2 Analysis of astrocyte purification by flow cytometry ..................................................... 40

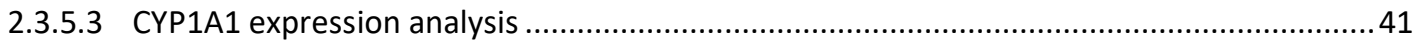

2.3.5.3.1 Evaluation of astrocyte-specific AhR deletion by FICZ treatment in vitro ................4 41

2.3.5.3.2 Activation of AhR transcriptional activity by FICZ or LAQ treatment in vitro ............41

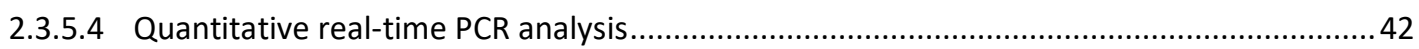

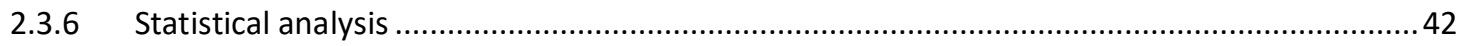


3.1 Part 1: The role of astrocytic and microglial AhR in CNS demyelination ........................... 43

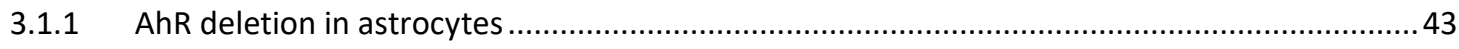

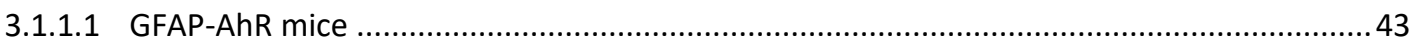

3.1.1.1.1 Expression of the AhR transcriptional target CYP1A1 is decreased in GFAP-AhR deficient astrocytes after FICZ stimulation

3.1.1.1.2 Clinical observations in GFAP-AhR knockout mice during cuprizone-induced demyelination.

3.1.1.1.3 GFAP-AhR knockout mice do not show any alteration in the extent of demyelination and the density of mature oligodendrocytes

3.1.1.1.4 No change in microglial activation and acute axonal damage in GFAP-AhR mice

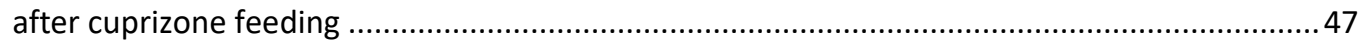

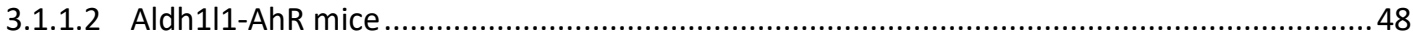

3.1.1.2.1 CYP1A1 mRNA levels are reduced in stimulated AhR-deleted Aldh1l1+ astrocytes .. 48

3.1.1.2.2 No clinical signs or body weight abnormalities of Aldh1l1-AhR conditional knockout mice 49

3.1.1.2.3 Cuprizone intoxication for 6 weeks does not modify the extent of demyelination and numbers of mature oligodendrocytes in Aldh1l1-AhR mice

3.1.1.2.4 Microglial density and acute axonal damage are unaltered in Aldh1l1-AhR mice compared to control mice

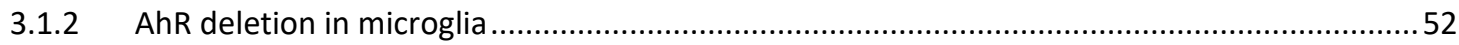

3.1.2.1 Clinical evaluation of CX3CR1-AhR conditional knockout mice .........................................52

3.1.2.2 Microglia-specific AhR deletion has no influence on demyelination but on the density of mature oligodendrocytes after 6 weeks of cuprizone treatment.

3.1.2.3 Microglia-specific AhR deletion does not affect microglial activation or acute axonal damage during cuprizone intake

3.1.2.4 Cuprizone challenge for 3 weeks reveals a higher density of mature oligodendrocytes and less demyelination in CX3CR1-AhR mice.....

3.1.2.5 No alteration in acute axonal injury but less microglial activation in CX3CR1-AhR mice after 3 weeks of cuprizone treatment

3.1.2.6 Microglial CYP1A1 is increased after FICZ treatment in vitro

3.2 Part 2: The role of LAQ as AhR ligand during cuprizone-induced demyelination.

3.2.1 Effects of astrocyte- and microglia-specific AhR deletion on LAQ efficacy in vivo .59

3.2.1.1 LAQ inhibits cuprizone-induced pathology independent of astrocytic AhR. 59

3.2.1.2 LAQ reduces demyelination, microglial activation, acute axonal injury and loss of mature oligodendrocytes independent of AhR deletion in Aldh $1 / 1^{+}$astrocytes 61

3.2.1.3 LAQ prevents cuprizone-induced pathology independent of microglial AhR ..... 


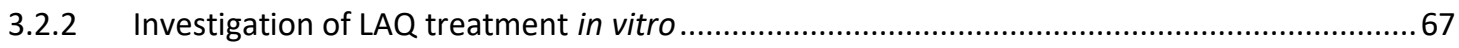

3.2.2.1 Astrocytic NF-KB activation is reduced by LAQ independent of AhR ............................67

3.2.2.2 LAQ does not induce AhR downstream genes in astrocytes .........................................68

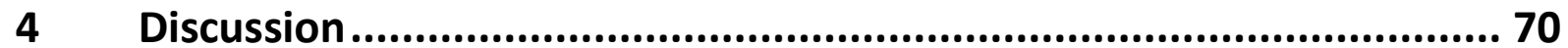

4.1 The AhR is expressed by astrocytes and microglia and upregulated in CNS inflammation . 71

4.2 AhR loss on astrocytes or microglia does not modify cuprizone-induced demyelination ... 72

4.3 Astrocytic NF-KB activation is reduced by LAQ independent of AhR ............................ 75

4.4 The role and function of astrocytes during cuprizone-induced demyelination ............... 77

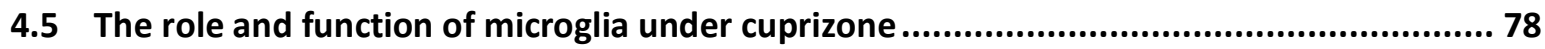

4.6 Outlook........................................................................................... 81

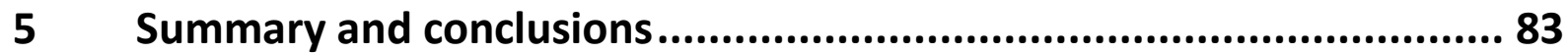

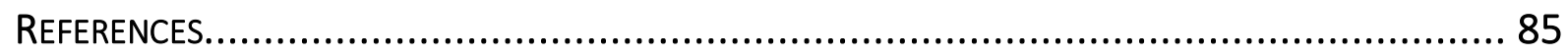

CURRICULUM VITAE 


\section{ACKNOWLEDGEMENTS}

First, I would like to thank my supervisor Prof. Dr. Wolfgang Brück for giving me the opportunity to work in his lab. I would also like to thank him for the supervision of this project and for scientific advice and discussions. I am also greatly indebted to Prof. Dr. Christine Stadelmann-Nessler for co-supervising my project, for her constant support, scientific discussions and help especially during the last year. I am particularly grateful for her scientific as well as personal advice. I would also like to thank Dr. Nadine Kramann for all her help and for sharing her scientific expertise with me.

I want to thank Prof. Dr. Alexander Flügel for agreeing to act as second examiner and for his supporting comments and ideas on my progress reports. I also want to acknowledge Prof. Dr. Peter Burfeind for his interest in my project and for his supportive contributions as third member of my thesis committee. Further thanks are also due to the additional members of my examination board, Prof. Dr. Dr. Hannelore Ehrenreich, Prof. Dr. Heidi Hahn and Prof. Dr. Frauke Alves.

I would like to especially thank Dr. Stefan Nessler, Dr. Franziska van der Meer and Dr. Anne Winkler for scientific discussions, methodological advice, and for their support.

I am also grateful to Dr. Franziska van der Meer, Dr. Silke Häusser-Kinzel, Dr. Anne Winkler, Dr. Neal Hermer and Cynthia Bunker for proofreading of this thesis.

Many thanks to laboratory assistants Heidi, Katja, Brigitte, Elke, Sarah, Susi, Olga and Uta for their theoretical and practical support in the experiments as well as for the pleasant conversations and the enjoyable atmosphere in the lab.

Furthermore, I am grateful to Sebastian, Erika and Carolin for making it possible for me to have several weekends off.

I owe many thanks to Franziska, Anne, Silke, Jana, Carolin, Sebastian, Anastasia, Darius, Adriane, Marie, Jasmin, Claudia, Patrik, Erika, Leila, Kim, Melanie and of course all other colleagues for the time we have spent together and for having lots of fun not only in the lab. I enjoyed the time I spent with you very much! 
I want to acknowledge the Molecular Medicine PhD program, and I would like to thank our always friendly secretaries Heidi, Gisela and Cynthia for administrative support and Bernd for the IT support.

I want to thank my family and friends in Munich and Göttingen, especially Steffi, for being there for me over all these years. Thank you for your constant support and unfailing confidence in me. Special thanks to my team from the standard formation TSC Schwarz-Gold Göttingen A, for making my time in Göttingen to an incredible and unforgettable stage of my life. The long and intensive time we spent together went far beyond "just jointly dancing".

\section{\#happytogether \#weiterimmerweiter}

Finally, I would like to thank my boyfriend Manuel for all his support during all these years, for his motivation, trust, endless patience and for believing in me. 


\section{ABSTRACT}

The aryl hydrocarbon receptor (AhR) is a ligand-activated transcription factor which plays a key role in the regulation of immune and inflammatory responses. AhR expression in astrocytes and microglia was shown to limit central nervous system (CNS) inflammation. However, little is known about the function of AhR signaling in glial cells. The aim of the present study was to evaluate the role of astrocytic and microglial AhR in CNS demyelination and to investigate the impact of cell-specific AhR deletion on the therapeutic efficacy of laquinimod (LAQ) under cuprizone.

Using the cuprizone mouse model of toxic demyelination, GFAP-, Aldh1l1- and CX3CR1AhR mice were evaluated histologically after 6 weeks of $0.25 \%$ cuprizone feeding. Cell-specific deletion of AhR was confirmed by in vitro expression analysis upon stimulation with the AhRspecific ligand FICZ. Mice lacking astrocytic AhR showed a similar number of mature oligodendrocytes compared to littermate controls, while animals with AhR-deleted microglia displayed a significantly higher oligodendrocyte density after cuprizone challenge. Furthermore, cuprizone feeding for 3 weeks revealed more mature oligodendrocytes and less demyelination in CX3CR1-AhR mice. However, cuprizone-induced demyelination, microglial activation and acute axonal damage in the corpus callosum were comparable in all mouse strains and Cre-negative controls after 6 weeks of cuprizone intake. To investigate the effects of astrocyte- and microglia-specific AhR deletion on LAQ efficacy during toxic demyelination, cuprizone-fed mice were treated simultaneously with LAQ. As in control animals, administration of LAQ prevented mature oligodendrocyte apoptosis, demyelination, microglial infiltration and acute axonal damage in all three mouse strains. Using a reporterbased in vitro assay, NF-KB activation was significantly reduced in AhR-deficient astrocytes as compared to controls. Astrocytic NF-KB activity was diminished by LAQ independent of AhR.

In conclusion, these data indicate that astrocytic and microglial AhR expression does not play a major role in mediating the cytotoxic effects of cuprizone treatment. Also, I demonstrate here that the efficacy of LAQ during toxic demyelination is independent of glial AhR expression. Nevertheless, given the fact that LAQ suppresses experimental autoimmune encephalomyelitis in an AhR-dependent manner, AhR signaling may offer a potential target for future therapeutic approaches, particularly for inflammatory diseases of the CNS. 


\section{LIST OF FIGURES}

Figure 1: Structural formula of the active substance Laquinimod sodium. .15

Figure 2: Time schedule of cuprizone experiments 35

Figure 3: High astrocyte purity after MACS cell separation ..... 43

Figure 4: Loss of AhR in GFAP-positive astrocytes leads to decreased CYP1A1 response upon

FICZ stimulation. .44

Figure 5: No significant difference in body weights of GFAP-AhR mice fed with $0.25 \%$ cuprizone for 6 weeks compared to control mice

Figure 6: Cuprizone feeding for 6 weeks induces strong demyelination with comparable numbers of mature oligodendrocytes in both GFAP-AhR and control mice .46

Figure 7: Extensive accumulation of microglia and axonal spheroids in the corpora callosa of both GFAP-AhR and control mice during demyelination

Figure 8: Reduced CYP1A1 mRNA levels in Aldh1l1+ astrocytes compared to control astrocytes after FICZ stimulation (in vitro)

Figure 9: No alteration in body weights of Aldh1l1-AhR compared to control mice after cuprizone intoxication 50

Figure 10: No change in demyelination or densities of mature oligodendrocytes between Aldh1l1-AhR and control mice

Figure 11: Numbers of $\mathrm{Mac3}^{+}$activated microglia and $\mathrm{APP}^{+}$axonal spheroids are comparable in the corpus callosum of Aldh1l1-AhR mutants and littermate controls after 6 weeks of cuprizone exposure

Figure 12: Body weights of CX3CR1-AhR mice are unaffected by cuprizone intoxication compared to control mice

Figure 13: Cuprizone treatment does not modify the extent of demyelination but leads to higher numbers of mature oligodendrocytes in CX3CR1-AhR mice versus littermate controls

Figure 14: Microglia-specific AhR deletion has no effect on acute axonal damage or microglial infiltration into the corpus callosum

Figure 15: 3 weeks of cuprizone challenge reveals less demyelination and slightly higher numbers of mature oligodendrocytes in CX3CR1-AhR mice compared to control animals 
Figure 16: Microglia-specific AhR deletion has no effect on acute axonal injury but decreases microglial activation after 3 weeks of cuprizone intake .57

Figure 17: Increased CYP1A1 expression in FICZ-treated control microglia 58

Figure 18: No weight loss in LAQ-treated GFAP-AhR mice after cuprizone exposure. 59

Figure 19: LAQ reduces demyelination, microglial activation, acute axonal damage and loss of mature oligodendrocytes after 6 weeks of cuprizone independent of astrocytic AhR

Figure 20: LAQ-treated Aldh1I1-AhR mice show constantly higher body weights than vehicletreated animals during cuprizone-induced demyelination

Figure 21: LAQ reduces cuprizone-induced demyelination, microglial infiltration, acute axonal damage and loss of mature oligodendrocytes regardless of AhR deletion in Aldh1l1-positive astrocytes

Figure 22: CX3CR1-AhR mice show significantly higher body weights after LAQ treatment compared to vehicle-treated mice during cuprizone intake.

Figure 23: LAQ prevents cuprizone-induced demyelination, microglial activation and acute axonal damage and rescues the density of mature oligodendrocytes independent of microglial AhR

Figure 24: LAQ attenuates astrocytic NF-KB activation independent of astrocytic AhR deficiency..... .68

Figure 25: LAQ does not increase CYP1A1 mRNA levels in wild type astrocytes. .69 


\section{LIST OF TABLES}

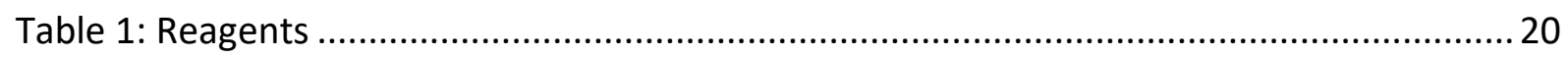

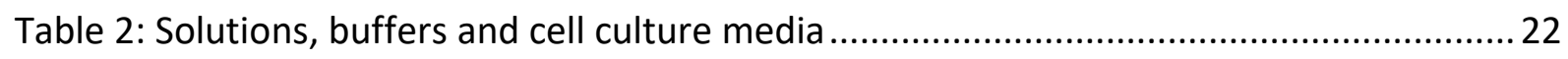

Table 3: Solutions and buffers for histochemistry and immunohistochemistry .................... 22

Table 4: Primary antibodies for immunohistochemical stainings ......................................... 23

Table 5: Secondary antibodies for immunohistochemical stainings .....................................24

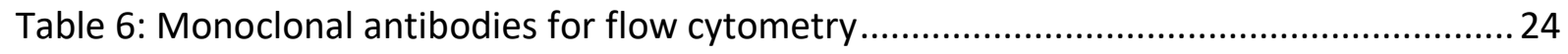

Table 7: Monoclonal antibody for magnetic-activated cell sorting ....................................... 24

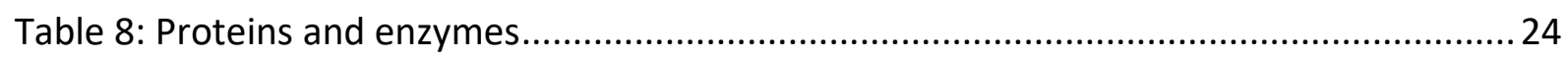

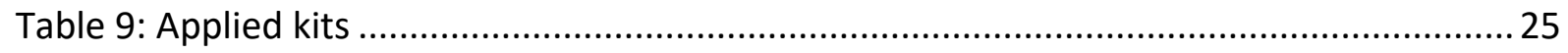

Table 10: Oligonucleotide primers used for transgenic mice genotyping .............................25

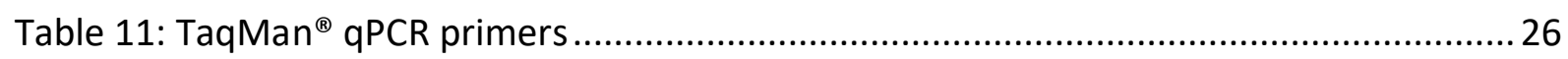

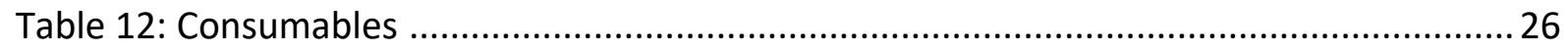

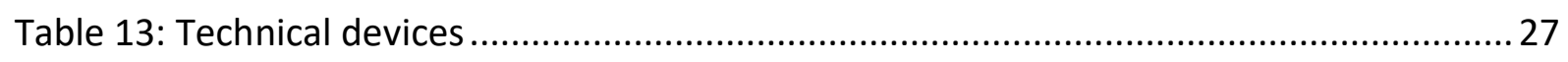

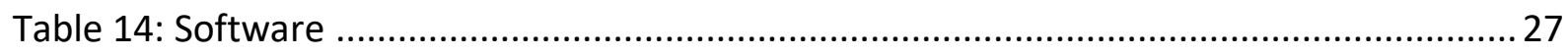

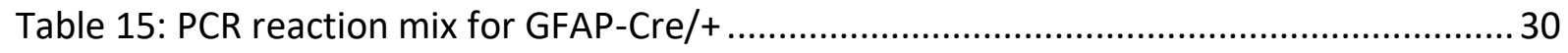

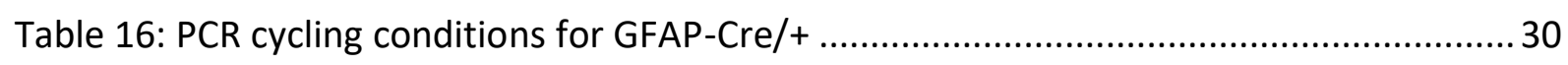

Table 17: PCR reaction mix for Aldh1l1-CreERT2/+ ............................................................ 31

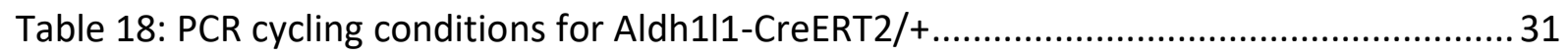

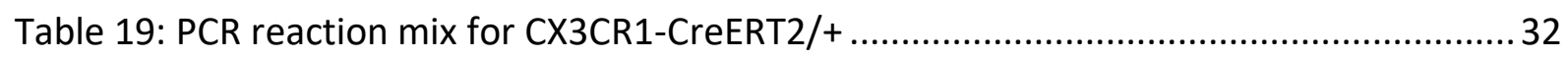

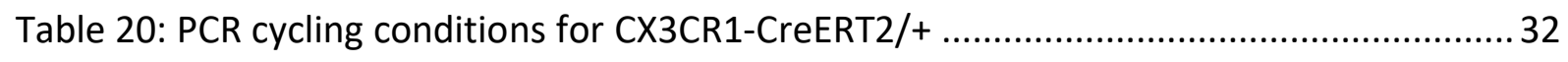

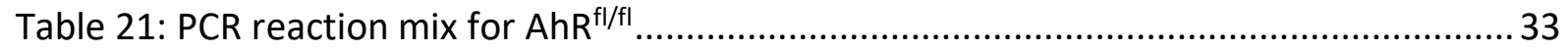

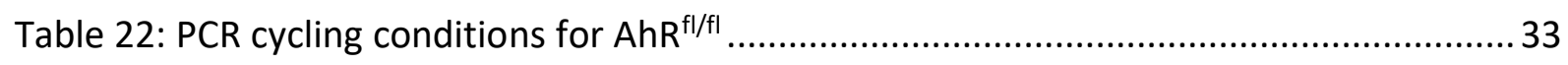

Table 23: Deparaffinization and rehydration steps ............................................................ 36 


\section{AbBreviations}

$\% \quad$ Per cent

${ }^{\circ} \mathrm{C} \quad$ Degrees Celsius

Ab Antibody/antibodies

ACSA-2 Astrocyte cell surface antigen-2

AhR Aryl hydrocarbon receptor

Aldh1l1 Aldehyde Dehydrogenase 1 Family Member L1

ANOVA Analysis of variance

APC Allophycocyanin

APC-Cy7 Allophycocyanin cyanine 7

APP Amyloid precursor protein

ARNT AhR nuclear translocator

B2m Beta-2 microglobulin

BBB Blood-brain barrier

BDNF Brain-derived neurotrophic factor

bHLH Basic helix-loop-helix

Bp $\quad$ Base pairs

BSA Bovine serum albumin

CD Cluster of differentiation

cDNA Complementary deoxyribonucleic acid

CNS Central nervous system

Com Common

CSF1R Colony-stimulating factor 1 receptor 
CT

Cycle threshold

CX3CR1 Chemokine (CX3C motif) receptor 1

CXCL10 C-X-C motif chemokine ligand 10

Cy5.5 Cyanine 5.5

CYP1A1 Cytochrome P450, family 1, subfamily A, polypeptide 1

CYP1A2 Cytochrome P450, family 1, subfamily A, polypeptide 2

Da Dalton

DAB 3,3'-Diaminobenzidine

DAPI 4',6-Diamidino-2-phenylindole

DMEM Dulbecco's Modified Eagle's Medium

DMSO Dimethyl sulfoxide

DNA Deoxyribonucleic acid

DNase Deoxyribonuclease

dNTP Deoxyribonucleoside triphosphate

e.g. Exempli gratia

EAE Experimental autoimmune encephalomyelitis

EDSS Expanded disability status scale

EDTA Ethylenediaminetetraacetic acid

ESR Oestrogen receptor

FACS Fluorescence-activated cell sorting

FCS Fetal calf serum

FICZ 6-Formylindolo[3,2-b]carbazole

FITC Fluorescein isothiocyanate

For $\quad$ Forward 
g

GAPDH Glyceraldehyde-3-phosphate dehydrogenase

GFAP Glial fibrillary acidic protein

GLAST Glutamate aspartate transporter

$\mathrm{h}$

H\&E Hematoxylin and eosin

$\mathrm{H}_{2} \mathrm{O}_{2} \quad$ Hydrogen peroxide

HBSS Hanks' buffered salt solution

$\mathrm{HCl} \quad$ Hydrochloric acid

i.e. Id est

i.p. Intraperitoneal

IFNץ Interferon gamma

IgG Immunoglobulin $\mathrm{G}$

IHC Immunohistochemistry

IKK2 IkB kinase 2

IL-1 $\quad$ Interleukin 1 beta

kDa $\quad$ Kilodalton

I L Liter

LAQ Laquinimod

LFB-PAS Luxol Fast Blue-Periodic Acid Schiff

LPS Lipopolysaccharide

M Molar

Mac Microglia/macrophage

MACS Magnetic-activated cell sorting 


\begin{tabular}{|c|c|}
\hline MBP & Myelin basic protein \\
\hline $\mathrm{mg}$ & Microgram \\
\hline $\mathrm{MgCl}_{2}$ & Magnesium chloride \\
\hline $\mathrm{MHC}$ & Major histocompatibility complex \\
\hline $\min$ & Minute(s) \\
\hline $\mathrm{ml}$ & Milliliter \\
\hline $\mathrm{mm}$ & Millimeter \\
\hline $\mathrm{mM}$ & Millimolar \\
\hline MOG & Myelin oligodendrocyte glycoprotein \\
\hline MRI & Magnetic resonance imaging \\
\hline mRNA & Messenger ribonucleic acid \\
\hline MS & Multiple Sclerosis \\
\hline $\mathrm{n}$ & Number of independent samples or animals \\
\hline $\mathrm{NaOH}$ & Sodium hydroxide \\
\hline NF-KB & Nuclear factor kappa-light-chain-enhancer of activated B cells \\
\hline ng & Nanogram \\
\hline NK cell & Natural killer cell \\
\hline $\mathrm{nm}$ & Nanometer \\
\hline Nrf2 & Nuclear factor erythroid 2-related factor 2 \\
\hline OPC & Oligodendrocyte precursor cell \\
\hline $\mathrm{P}$ & Postnatal day \\
\hline P25 & Brain-specific $25 \mathrm{kDa}$ protein \\
\hline p65 & RelA, subunit of NF-KB \\
\hline PAS & PER-Arnt-SIM \\
\hline
\end{tabular}




\begin{tabular}{|c|c|}
\hline PBS & Phosphate buffered saline \\
\hline PCR & Polymerase chain reaction \\
\hline PE & Phycoerythrin \\
\hline PerCP & Peridinin chlorophyll protein \\
\hline PFA & Paraformaldehyde \\
\hline PLL & Poly-L-Lysin \\
\hline PLP & Proteolipid protein \\
\hline pmol & Picomole \\
\hline POX & Streptavidin-horseradish peroxidase \\
\hline PPMS & Primary progressive multiple sclerosis \\
\hline qRT-PCR & Quantitative real-time polymerase chain reaction \\
\hline Rev & Reverse \\
\hline RNA & Ribonucleic acid \\
\hline RRMS & Relapsing-remitting multiple sclerosis \\
\hline RT & Room temperature \\
\hline s & Second(s) \\
\hline SD & Standard deviation \\
\hline SDS & Sodium dodecyl sulfate \\
\hline SEM & Standard error of the mean \\
\hline SOCS2 & Suppressor of cytokine signaling 2 \\
\hline SPMS & Secondary progressive MS \\
\hline TBE & Tris/borate/EDTA \\
\hline TCDD & $2,3,7,8$-Tetrachlorodibenzo-p-dioxin \\
\hline TGF $\alpha$ & Transforming growth factor alpha \\
\hline
\end{tabular}




\begin{tabular}{|c|c|}
\hline Th1 & T helper type 1 cells \\
\hline Th17 & T helper 17 cells \\
\hline TLR & Toll-like receptor \\
\hline TNF- $\alpha$ & Tumor necrosis factor alpha \\
\hline $\mathrm{TPPP} / \mathrm{p} 25$ & Tubulin polymerization promoting protein/ $25 \mathrm{kDa}$ brain-specific protein \\
\hline $\mathrm{T}_{\text {reg }}$ cell & Regulatory T cell \\
\hline Tris & Tris(hydroxymethyl)aminomethane \\
\hline $\mathrm{U}$ & Units \\
\hline UV & Ultraviolet \\
\hline V & Volt \\
\hline VEGF-B & Vascular endothelial growth factor B \\
\hline Veh & Vehicle \\
\hline WT & Wild type \\
\hline$\mu g$ & Microgram \\
\hline$\mu l$ & Microliter \\
\hline$\mu \mathrm{m}$ & Micrometer \\
\hline$\mu \mathrm{M}$ & Micromolar \\
\hline
\end{tabular}




\section{INTRODUCTION}

\subsection{Multiple Sclerosis}

Multiple Sclerosis (MS) is the most common chronic inflammatory, demyelinating and neurodegenerative disease of the central nervous system. It is characterized by fully or partially reversible episodes of neurological deficits (Filippi et al. 2018). MS, affecting more than 2 million people worldwide, is still not yet sufficiently understood, particularly with regards to etiology and epidemiology (Reich et al. 2018). Epidemiological data indicate that environmental factors including smoking, obesity, infection with the Epstein-Barr virus (EBV), low vitamin D levels or ultraviolet B light (UVB) exposure, together with an individual's genetic background play an important role in MS development (Ramagopalan et al. 2010). To date, diverse disease-modifying therapies are available but no medication fully prevents or reverses the heterogeneous presentation of clinical symptoms and disease progression. The pathological hallmark of MS is the formation of lesions in the brain and spinal cord due to immune cell infiltration. These lesions are characterized by demyelination, inflammation and glial reaction (Reich et al. 2018). While MS is thought to be an organ-specific autoimmune disorder (Dobson and Giovannoni 2019), glial cells such as astrocytes and microglia carry complex but important functions in either promoting tissue injury or repair (Voet et al. 2019; Ponath et al. 2018).

\subsubsection{Symptoms, diagnosis and clinical course}

Although the clinical phenotype and course of MS is heterogeneous and depends on the location of damage, the so-called lesion, there are some highly representative features of the disease. Typically, the onset of MS is between 20 years and 40 years of age, with higher prevalence in women, and is characterized by a clinical attack that lasts for at least 24 hours up to weeks. These episodes are known as relapses and induce fully or partially reversible symptoms (Filippi et al. 2018). Optic neuritis, which causes partial or total visual loss in one eye, is the first symptom in $25 \%$ of patients and in general occurs in $70 \%$ of cases (Toosy et al. 2014). Initial symptoms can also be sensory disturbances such as numbness and tingling or motor disorders. Motor impairments affect almost all patients during the course of the disease and include muscle weakness, walking difficulties and spasticity. Further common symptoms 
are cognitive deficits, fatigue, bladder dysfunction, bowel impairment, sexual problems, emotional changes, dizziness, vertigo and affective disturbances such as depression or pain (Filippi et al. 2018).

The diagnosis of MS is evaluated using the McDonald criteria, which were originally introduced in 2001 and last revised in 2018 (McDonald et al. 2001; Polman et al. 2011; Thompson et al. 2018). These criteria include magnetic resonance imaging (MRI) analysis to identify the dissemination of demyelinating lesions in space or over time (dissemination in space (DIS), dissemination in time (DIT)) and to monitor disease activity. Further parameters are the verification of cerebrospinal fluid-specific oligoclonal bands and the measurement of clinic disability using the expanded disability status scale (EDSS).

Generally, there are four different disease courses of MS: relapsing-remitting (RR), secondary progressive (SP), primary progressive (PP), and progressive relapsing (PR) MS (Lublin and Reingold 1996). 85\% of MS patients present with a first episode of neurological dysfunction, which is designated as a clinically isolated syndrome (CIS) showing features of a single white matter lesion and concomitant inflammatory demyelination. The likelihood that a CIS evolves into clinically definite MS is high (Miller et al. 2005). About $85-90 \%$ of MS patients develop a relapsing-remitting disease course, which is characterized by the occurrence of relapses at irregular intervals with complete or incomplete recovery between episodes (Brownlee et al. 2017; Lublin et al. 2014). Over time, most RRMS patients develop a progressive phenotype, referred to as SPMS, which is associated with irreversible disability. About $10-15 \%$ of all patients develop a progressive clinical course from disease onset that is termed PPMS. It is characterized by gradual and permanent worsening without relapses (Lublin and Reingold 1996; Filippi et al. 2018). The rarest form of relapsing-progressive MS is defined by progressive disease course from the onset with acute relapses and periods of steady deterioration (Filippi et al. 2018).

\subsubsection{Pathology}

The pathological hallmarks of MS are a breakdown of the blood-brain barrier (BBB) and lesions in both white and grey matter of the CNS and spinal cord (Filippi et al. 2018). Focal demyelinated lesions in the white matter, known as plaques, are randomly distributed within the CNS, although frequently located in periventricular and subcortical white matter, optic 
nerves, cerebellar white matter, pons and medulla, the spinal cord and in the cervical portions (Kutzelnigg and Lassmann 2014). Lesions are characterized by inflammation-induced demyelination, accompanied by activated microglia, astrogliosis and axonal damage (Filippi et al. 2018; Prineas 1985; Brück and Stadelmann 2005).

In the last 25 years, a number of classification systems for lesion types have been introduced (Bo et al. 1994; Trapp et al. 1998; Lucchinetti 2000; van Waesberghe et al. 1999; Ferguson et al. 1997; Brück 2005; Brück et al. 1995). The latest by Kuhlmann et al. provides an updated histological classification system for MS lesions based on inflammatory and demyelinating activity; plaques are categorized into active, mixed active/inactive, and inactive lesions with or without ongoing demyelination (Kuhlmann et al. 2017). While active lesions are defined by the presence of macrophages/microglia throughout the lesion area, mixed active/inactive lesions are characterized by a hypocellular lesion center and macrophages/microglia confined to the lesion border. Inactive lesions occur more frequently in progressive stages of MS (Frischer et al. 2015). They are clearly demarcated without macrophage/microglia rim at the lesion border and are characterized by almost complete loss of macrophages/microglia and mature oligodendrocytes, distinct loss of axons and myelin, low density of T and B cells and reactive astrogliosis (Lassmann et al. 2012; Kuhlmann et al. 2017).

A distinctive feature in patients with MS are cortical lesions (Calabrese et al. 2012), which comprise three different types: leukocortical lesions encompassing deep cortical areas and subcortical white matter (type 1), intracortical lesions centered on intracortical microvessels (type 2) and subpial lesions extending from the pia mater into the superficial cortical layers (type 3) (Peterson et al. 2001). Grey matter lesions show a lower degree of inflammation and less microglia/macrophage recruitment compared to white matter lesions (Albert et al. 2007; Kutzelnigg et al. 2005; Peterson et al. 2001).

\subsubsection{Pathogenesis}

Both the adaptive and the innate immune systems are involved in MS initiation and progression. The highly conserved innate immune system is the first line of defense against pathogens and includes cellular barriers such as the blood-brain barrier (BBB) (Mayo et al. 2012). The BBB consists of endothelial cells, pericytes and their basal lamina with surrounding 
astrocytes and perivascular macrophages. In the healthy brain, the functions of the BBB include maintenance of brain homeostasis, protecting the CNS from damage and regulation of influx and efflux transport (Obermeier et al. 2013). In pathological conditions such as MS, the BBB is disrupted and accompanied by demyelination, oligodendrocyte and axonal loss, as well as inflammation (Ortiz et al. 2014).

It remains an enigma whether BBB dysfunction leads to immune cell infiltration or is a result of perivascular leukocyte aggregation. However, it was reported that leukocyte migration alters BBB permeability (Larochelle et al. 2011) and provokes the interaction of proinflammatory autoreactive $\left(\mathrm{CD}^{+}\right) \mathrm{T}$ cells with adhesion molecules on the capillary endothelium. As a consequence, T cells migrate across the BBB and enter the CNS. After reactivation by antigen-presenting cells, $T$ cells release pro-inflammatory cytokines that activate resident cells and recruit other immune cells, eventually leading to myelin and widespread axonal destruction (Lassmann et al. 2007; Sospedra and Martin 2005; Greter et al. 2005; Williams et al. 2007).

Although it is still not fully understood whether MS is initially triggered by CNS-intrinsic or -extrinsic processes, MS is predominantly considered a T cell-mediated disease. Active demyelinating lesions are infiltrated by a variable degree of cytotoxic $\left(C D 8^{+}\right)$T cells, few $C D 20^{+}$ $B$ cells, activated phagocytes, large and reactive astrocytes, immunoglobulin-containing plasma cells and few helper (CD4 $\left.{ }^{+}\right)$T cells (Machado-Santos et al. 2018; Popescu and Lucchinetti 2012).

Besides adaptive immune cells, innate immune cells such as dendritic cells, microglia and macrophages were also found in lesions and reported to play an important role in disease pathogenesis (Mayo et al. 2012). In MS patients, dendritic cells displayed an activated phenotype owing to enhanced expression of CD40 and CD80 and showed higher levels of proinflammatory cytokines such as IFN and TNF $\alpha$ which promote T cell responses. During the change from relapsing-remitting to a progressive MS stage, the profile of the cytokines as well as co-stimulatory molecule expression were found to be altered in dendritic cells (Karni et al. 2006; Weiner 2008; Gandhi et al. 2010). Compared to RRMS patients, patients with progressive MS displayed profound activation of microglial cells (Kutzelnigg et al. 2005). The main CNS-intrinsic immune effector cells microglia/macrophages contribute to MS 
pathogenesis and inflammation through phagocytosis of myelin debris, secretion of proinflammatory cytokines and antigen presentation (Benveniste 1997).

In the $19^{\text {th }}$ century, astrocytes were considered to be a kind of connective tissue (Virchow 1856). Today we know that astrocytes have many other functions. Astrocytes play a supportive role by controlling BBB permeability, maintaining extracellular homeostasis, and regulating synaptogenesis and neurotransmission (Wheeler and Quintana 2019). In the pathophysiology of MS, astrocytes have both protective as well as deleterious roles, i.e. by activating and terminating immune responses (Correale and Farez 2015). One classic example of an astrocytic response to CNS injury is astrogliosis, one of the pathological hallmarks of MS (Domingues et al. 2016).

\subsubsection{The role of astrocytes and microglia in CNS health and disease}

Our current knowledge of the role of astrocytes has changed tremendously over time. Historically, this type of glia was understood to be a relatively homogeneous cell population that holds the nervous system together; this is reflected in the etymology of the term itself, which is derived from the Greek and means glue (Virchow 1856; Domingues et al. 2016). Astrocytes were described as glial fibrillary acidic protein $\left(G F A P^{+}\right)$- expressing cells with either protoplasmic or fibrous morphology (Ramón Y Cajal 1909). Recent advances in astrocyte biology, however, indicate a far more complex heterogeneity. Based on function and morphology, astrocytes can be divided into four different types in the human brain: fibrous white matter astrocytes, protoplasmic grey matter astrocytes, as well as varicose and interlaminar astroglia (Vasile et al. 2017). In addition, there are specialized astroglia such as unipolar Bergmann glia in the cerebellum (De Zeeuw and Hoogland 2015). As the most abundant glial cell type of the CNS, astrocytes possess essential functions in maintaining the BBB, promoting neuronal survival by providing trophic, antioxidant and metabolic support to neurons, regulating synaptogenesis and synaptic transmissions, neurotransmitter recycling and controlling regional blood flow (Ben Haim and Rowitch 2017).

Astrocytes were reported to influence microglia, which are the main innate immune cells of the central nervous system. As sensors of pathologic changes, microglia are constantly occupied with surveilling their microenvironment, and are substantially involved in the clearance of dying cells, pathogens and molecules, thereby engaging pattern recognition 
receptors (Domingues et al. 2016). However, microglial activation can induce protective and regenerative as well as damaging responses. Based on their gene expression, it has been suggested that microglia be classified as pro-inflammatory M1 cells or anti-inflammatory M2 microglia (Lloyd and Miron 2019).

After CNS injury, microglia support remyelination through clearance of myelin debris via CX3CR1, induction of receptor expressed on myeloid cells 2 (TREM2) and colony-stimulating factor 1 receptor (CSF1R) signaling as well as removal of cholesterol via apolipoprotein E (APOE), LXR $\alpha, A B C A 1$ and ABCG1. In addition, microglia facilitate recruitment and differentiation of oligodendrocyte precursor cells (OPCs) by secretion of growth factors and cytokines such as activin A and insulin-like growth factor 1 (IGF1) and by modulating the extracellular matrix (Lloyd and Miron 2019). In MS, microglia change their transcriptional profile and turn into disease-associated microglia (DAM), which display a severe inflammatory phenotype (Voet et al. 2019). Moreover, microglia secrete pro-inflammatory molecules that might harm oligodendrocytes or their myelin sheaths, and interact as antigen-presenting cells with other immune cells, which results in T cell activation (Prinz and Priller 2014).

Similarly, astrocytes possess both protective and deleterious capacities in MS. In response to CNS damage and disease, astrocytes proliferate and form glial scars which consist of a dense network of hypertrophic reactive cells and processes. This response, referred to as astrogliosis, is accompanied by the upregulation of intermediate filaments such as GFAP and vimentin, and changes in gene expression of antigen presentation molecules, cytokines, receptors, growth factors, enzymes, protease inhibitors and adhesion molecules (Sofroniew 2014; Pekny and Pekna 2004; Williams et al. 2007). GFAP expression plays a crucial role in astrocyte cell signaling. In mouse studies of toxin-induced demyelination, constitutive expression of GFAP inhibited CXCL10 production and reduced NF-KB activation. This resulted in the protection of myelin, oligodendrocytes and axons and eventually disease suppression (Kramann et al. 2019). After acute CNS injury, LPS-induced neuroinflammation and in neurodegenerative diseases such as MS, activated microglia were found to induce a neurotoxic subtype of reactive astrocytes, termed A1 astrocytes. In contrast, ischemiainitiated $\mathrm{A} 2$ reactive astrocytes were assumed to be protective by promoting neuronal survival and tissue repair (Liddelow et al. 2017; Sofroniew and Vinters 2010). 
Astrocytes have been observed to activate immune responses by secreting proinflammatory cytokines (IL-1 $\beta$, IL-6, IL-12, IL-17, IL-23; TNF- $\alpha$ ) or terminate immune responses via anti-inflammatory cytokines (IL-10, TGF- $\beta$, IL-27) or microglial inhibition (Correale and Farez 2015). Moreover, astrocytes recruit immune cells to CNS lesion sites by chemokine production, regulation of the BBB integrity or modulation of adhesion molecule expression such as VCAM-1 (Mayo et al. 2012). Astrocytes and microglia express Toll-like receptors (TLRs) which play an essential role in mediating innate immune responses to exogenous pathogens. In MS, expression of astrocytic TLR3 was observed in brain and spinal cord (Bsibsi et al. 2002). Upon activation by pro-inflammatory cytokines, TLR3 or TLR4 agonists or oxidative stress, human astrocytes exhibited remarkably strong induction of TLR3, which boosted the production of anti-inflammatory cytokines such as IL-9, IL-10 and IL-11 (Bsibsi et al. 2006). In contrast, astrocytic TLR3 signaling triggered the secretion of pro-inflammatory cytokines including IL-12, TNF $\alpha$, IL-6, CXCL10 and IFN $\beta$ (Jack et al. 2005).

\subsection{The cuprizone mouse model}

MS is an autoimmune demyelinating disease of the central nervous system (CNS) with profound heterogeneity in terms of clinical course and pathogenesis (Brück 2005). Although there is no animal model that captures all facets of MS, several mouse models have been established, and each mimics parts of MS pathology, thus allowing the investigation of the complex underlying molecular mechanisms. The three most commonly studied animal models include experimental autoimmune encephalomyelitis (EAE), the cuprizone model and Theiler's viral model of inflammatory demyelination. In EAE, the most commonly used model for studying the inflammatory aspects of MS, mice are immunized with myelin antigens or receive a passive transfer of myelin-specific $T$ lymphocytes that leads to inflammatory demyelination of the CNS. In Theiler's virus model, animals are infected with the neurotropic picornavirus termed Theiler's murine encephalomyelitis virus (TMEV), resulting in a progressive $\mathrm{CD}^{+} \mathrm{T}$ cell-mediated demyelinating disease (Torkildsen 2008). In contrast, the cuprizone model is used to study the formation and progression of demyelinating CNS lesions (Kipp et al. 2017).

Cuprizone (bis-cyclohexanone oxaldihydrazone) is a chelator initially used as a reagent for copper analysis. First experiments were performed by Carlton in the late 1960s, where he used 
different concentrations of cuprizone ranging from 0.2 to $0.5 \%$ mixed in basic rodent chow. At the highest dose $(0.5 \%)$, he reported severe growth retardation, paresis of the posterior limbs and microscopic lesions in the brain accompanied by severe CNS pathology including hydrocephalus, edema, demyelination and astrogliosis (Carlton 1967). Particularly at these high concentrations, cuprizone was found to be toxic when given during early development, resulting in high mortality, status spongiosus, astrocytosis, oligodendrocyte degeneration and giant hepatic mitochondria (Suzuki 1969; Carlton 1967; Blakemore 1972).

The standard protocol today uses 8- to 10-week-old mice that for 5 to 6 weeks are fed a diet containing $0.2-0.25 \%$ cuprizone $(\mathrm{w} / \mathrm{w})$ in powdered standard rodent chow. This induces oligodendrocyte apoptosis with subsequent demyelination and microglial activation (Hiremath et al. 1998). Oligodendroglial cell death starts already after a few days of cuprizone intoxication and reaches its maximum between 10 and 21 days, whereas activated caspase- 3 can predominantly be detected in apoptotic oligodendrocytes between days 6 and 10 . Demyelination is immunohistochemically detectable after 3 weeks and is nearly complete in the corpus callosum at week 4.5-5 and in the cortex at week 6 (Matsushima 2001; Hesse et al. 2010; Gudi et al. 2009). Early remyelination was observed shortly after the peak of demyelination or few days after cuprizone withdrawal. Six weeks after cuprizone cessation, myelin loss was entirely compensated (Skripuletz et al. 2008). Although gradually decreased during the first weeks of recovery (Schultz et al. 2017), axonal damage was still detectable and considerably enhanced in long-term remyelinated mice compared to aged-matched controls (Manrique-Hoyos et al. 2012). In contrast to MS and EAE, the BBB is intact with the near absence of T lymphocytes (Bakker and Ludwin 1987; Hiremath et al. 1998). This allows the investigation of CNS-intrinsic effects without significant influence of the peripheral immune system.

Previous studies report a crucial role of astrocytic NF-KB activation for oligodendrocyte damage during toxic demyelination. Importantly, mice deficient in astrocytic, but not oligodendroglial, NF-KB activation under cuprizone showed strong preservation of CNS myelin, reduced glial response and expression of pro-inflammatory mediators (Raasch et al. 2011). Moreover, other studies could confirm reduced cuprizone-induced demyelination with decreased microglial and polydendrocyte cellular reactivity in mice lacking astrocytic adapter protein Act1, IL-17A or IL-17 receptor C (IL-17RC) (Kang et al. 2012). Recent findings indicate that sustained increase in astrocytic GFAP expression inhibits CXCL10 production and NF-KB 
activation, which resulted in the protection of myelin, oligodendrocytes and axons from cuprizone-induced demyelination (Kramann et al. 2019).

Further support for an astrocytic contribution in this model comes from loss-of-function studies based on astrocyte depletion during cuprizone challenge. Ablation of astrocytes does not prevent myelin damage but inhibits the removal of myelin debris. This demonstrates that astrocytes play a crucial role in myelin clearance via recruitment of microglia, suggesting key functions in the regulation of demyelinating processes of the CNS (Skripuletz et al. 2013).

Despite numerous hypotheses on the underlying mechanisms of oligodendrocyte damage, the exact mode of cuprizone action remains unknown (Gudi et al. 2014; Kipp et al. 2009). The underlying cause of oligodendrocyte damage was hypothesized to result from mitochondrial disturbances due to the appearance of enlarged mitochondria in liver and brain (Suzuki 1969; Hemm et al. 1971; Komoly et al. 1987), followed by enhanced production of oxidative agents, disturbed energy metabolism of oligodendrocytes and final cell death (Gudi et al. 2014). Taken together, the cuprizone model is a widely used non-invasive model that allows investigation of highly reproducible and reversible demyelination in white and grey matter of the CNS with an intact blood-brain barrier.

\subsection{The aryl hydrocarbon receptor}

The aryl hydrocarbon receptor (AhR) is a ligand-activated transcription factor that is a potential target for therapeutic approaches in autoimmune, neoplastic and degenerative diseases of the CNS (Rothhammer and Quintana 2019). Although AhR was initially recognized as a mediator of the toxicity of xenobiotics, it also plays an essential role in regulating immune responses (Stockinger et al. 2014). Depending on endogenous ligands such as dietary, microbial or metabolic signals, or exogenous ligands including environmental small molecules, the AhR controls diverse biological processes through ligand-specific, cell type-specific and context-specific mechanisms (Rothhammer and Quintana 2019).

\subsubsection{AhR expression in the body}

The AhR is widely expressed in the body, showing highest levels in the lung. High AhR expression, albeit 5-10 times lower than in the lungs, was also found in the murine brain, 
heart, liver and placenta (Li et al. 1994; Schmidt and Bradfield 1996). Since AhR is particularly expressed in the immune system, in addition to the lung, it is also abundantly expressed in barrier organs such as skin and gut (Stockinger et al. 2014). In the immune system, AhR is strongly expressed in Th17 cells and dendritic cells (Esser et al. 2009) and reported to be represented in macrophages, Langerhans cells, B cells, natural killer (NK) cells, innate

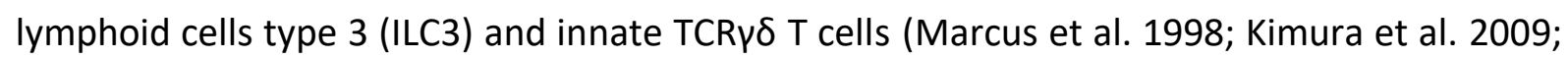
Stockinger et al. 2011; Qiu et al. 2012; Jux et al. 2009; Shin et al. 2013). Within the CNS, AhR expression was found in microglia, neurons and strongly represented in astrocytes in the brains of rodents (Lee et al. 2015; Rothhammer et al. 2016; Dever et al. 2016; Williamson et al. 2005). Although there is no evidence showing AhR expression in oligodendrocytes, the AhR was detected in the myelinating cells of the peripheral nervous system, the Schwann cells (Shackleford et al. 2018). Low levels of AhR were reported in spleen, kidney and muscle tissue (Li et al. 1994).

\subsubsection{Endogenous and exogenous ligands}

Besides many other exogenous agonists, the $A h R$ is most prominent for its role in binding and mediating the toxicity of xenobiotics such as 2,3,7,8-tetrachlorodibenzo-p-dioxin (TCDD). In 1976, a tragic industrial accident took place in the Italian city Seveso. During a chemical explosion, the population and environment of Seveso were exposed to up to 30 kilograms of the organic pollutant TCDD. TCDD is a highly persistent contaminant, known carcinogen and endocrine disruptor, which is commonly produced as a side product of industrial and combustive processes (Warner et al. 2014; di Domenico et al. 1980). As a result, chloracne outbreaks and life-threatening symptoms such as renal and progressive liver failure, emphysema, and myocardial degeneration occurred in chemical workers and residents (Stockinger et al. 2014; Caramaschi et al. 1981). In addition to the prototypical dioxin TCDD, further high-affinity AhR ligands of halogenated aromatic hydrocarbons (HAHs) include dibenzofurans and biphenyls. Other exogenous factors, albeit with lower affinity, are polycyclic aromatic hydrocarbons (PAHs) (such as 3-methylcholanthrene, benzo(a)pyrene, benzanthracenes and benzoflavones), but also nonclassical ligands such as nicotine were reported to activate the AhR (Denison and Nagy 2003; Iba et al. 1998). 
Apart from chemicals, a range of endogenous ligands have been identified, including dietary, commensal microflora and physiological compounds. Dietary components derived from cruciferous vegetables are converted to AhR ligands ICZ (indolo-[3,2-b]-carbazole) and DIM (3,3'-diindolylmethane) under acidic conditions in the stomach. Another physiological source of AhR agonists is the essential amino acid tryptophan, which must be obtained from the diet. Tryptophan is degraded by bacterial or enzymatical processes, resulting in AhRactivating metabolites such as indoles, kynurenine, indole-3-acetic acid (IAA) and 5-hydroxytryptamine (5-HT) (Stockinger et al. 2014). Upon UV irradiation, 6-formylindolo[3,2b]carbazole (FICZ) has been identified as a photooxidation product of tryptophan that binds AhR with high affinity comparable to that of TCDD (Rannug et al. 1987). Furthermore, mice treated with the synthetic tryptophan metabolite 3,4-DAA revealed ameliorated EAE disease course (Platten et al. 2005).

\subsubsection{Signaling pathway}

The AhR belongs to the family of basic helix-loop-helix (bHLH) PAS domain transcription factors, which consist of the following three functional domains: bHLH, PAS and a Q-rich domain. The bHLH motif is associated with DNA binding and protein-protein interaction. The PER-Arnt-SIM (PAS) domain, present twice, is responsible for ligand binding and proteinprotein interactions, and comprises three proteins: period circadian protein (PER), AhR nuclear translocator (ARNT) and single-minded protein (SIM). The third component is a proline-rich domain, which is involved in transcriptional activation (Stockinger et al. 2014).

The AhR is located in the cytoplasm and is bound to actin filaments as an inactive complex, together with chaperone proteins including heat shock protein 90 (HSP90), protein kinase SCR, AhR-interacting protein (AIP) and cochaperone p23. Upon agonist binding, the AhR complex is released from actin filaments and translocates into the nucleus. In order to separate from the chaperone complex, the AhR heterodimerizes with ARNT and finally binds genomic regions that contain the dioxin or xenobiotic response element (DRE/XRE) motif. This induces downstream transcription of target genes AhR repressor (Ahrr), indoleamine 2,3-dioxygenase 1 (Ido1), tryptophan-2,3-dioxygenase (Tdo) and cytochrome P450 enzymes such as Cyp1a1 and Cyp1b1. AhR signaling is controlled at three levels: by proteasomal degradation of AhR, AhRR-induced AhR/ARNT complex disruption or by CYP1A1-mediated ligand metabolism. In 
additional signaling pathways, AhR has been described to interact with further transcription factors and to control expression of genes that do not harbor xenobiotic response elements. AhR can also control the activation of pro-inflammatory nuclear factor kappa-light-chainenhancer of activated B cells (NF-KB) in direct or indirect ways. Moreover, the ligand-activated AhR-ARNT heterodimer has been reported to interact with oestrogen receptor (ESR) leading to the recruitment of the AhR-ESR complex to oestrogen-responsive gene promoters in the absence of ESR ligands (Rothhammer and Quintana 2019; Ohtake et al. 2003).

\subsubsection{Function}

The ligand-dependent transcription factor AhR is best known for its role in mediating the metabolism and detoxification of environmental chemicals. However, extensive research on AhR led to a paradigm shift toward its role as a regulator of inflammation. Today, AhR has been identified as a notable regulator of cell development, differentiation and function (Esser and Rannug 2015). Due to its high expression in barrier organs, AhR is an important environmental sensor that controls the adaptation to the cellular environment by sensing oxygen levels, redox potential and changes in the circadian rhythm (Rothhammer and Quintana 2019). Moreover, studies from AhR knockout mice provide evidence that the AhR carries important endogenous functions such as control in perinatal growth, hepatic and vascular development, fertility, peripheral and intestinal immunity, hematopoietic stem cells, as well as in cancer (Esser 2009; Singh et al. 2009; Safe and McDougal 2002; Esser and Rannug 2015). Depending on the cellular context, AhR ligands affect several pathways. Thus, the AhR plays an essential role in the regulation of autoimmune inflammatory diseases of the central nervous system (MS), gut (e.g. Crohn's disease and ulcerative colitis), connective tissue (rheumatoid arthritis) and the skin (psoriasis) (Nguyen et al. 2013; Colonna 2014; Monteleone et al. 2011; Rothhammer et al. 2016).

\subsubsection{Constitutive AhR knockout animals}

To understand the role of AhR in the toxicity of TCDD, but also to elucidate its endogenous functions, three different AhR null mice were generated independently and almost simultaneously in the 1990s. A Japanese group replaced part of exon 1 of the AhR locus with the bacterial $\beta$-galactosidase gene (Mimura et al. 1997). These mice, however, displayed a 
postnatal lethality of $50 \%$. In Spanish laboratories, exon 1 was substituted from the translational start site onwards with a neomycin gene instead (Fernandez-Salguero et al. 1995). Similar to the previously mentioned animals, $40-50 \%$ of the mice died after birth. This knockout strain was the only one with an obvious immune phenotype, such as significantly decreased numbers of splenic $T$ and B cells or skin inflammation (Fernandez-Salguero et al. 1997). The most widely used AhR null mutant mice (B6.129-AhR $R^{\text {tm1Bra } / J ~ S t o c k ~ 002831, ~ a v a i l a b l e ~}$ via Jackson Laboratories) were developed by the US group of Christopher Bradfield who targeted exon 2, which encodes the basic helix-loop-helix domain that is associated with DNA recognition and dimerization with ARNT (Schmidt et al. 1996). This knockout strain is characterized by no postnatal lethality but other immune and hepatic defects such as thinner periarterial lymphoid sheaths and impaired fatty metamorphosis. AhR-deficient mice of all three genetically engineered null mutant mice presented vascular abnormalities such as patent ductus venosus and consequential liver phenotypes including decreased size, portal fibrosis and steatosis, as well as defects in reproduction, growth retardation and failure to induce CYP1A1 and CYP1A2 (Lahvis and Bradfield 1998; Esser 2009). Lastly, a fourth knockout strain termed C57BL/6-Ahrtm1.2Arte has been generated by Taconic Biosciences (Taconic). These animals carry a deletion in exon 3 of the AhR gene, which leads to an out-of-frame splicing of exons 2 to exon 4. Furthermore, these AhR null mouse mutants are also smaller than heterozygotes or wild types and show immune system abnormalities as well as frequent death of newborns or missing litters, with some incidence of death after weaning.

\subsubsection{AhR in the CNS}

Glia comprise 50 to $90 \%$ of cells within the central nervous system (von Bartheld et al. 2016). Two glial lineages, microglia and astrocytes, are implicated as important drivers of CNS innate immune responses (Sofroniew 2009; Goldmann and Prinz 2013). Their wide-ranging distribution and numerous crucial functions suggest these cells as candidates for sensing and coordinating the response to several metabolic, microbial and environmental stimuli such as AhR ligands. However, on the basis of currently available information, the relevance of AhR signaling in glial cells is limited.

Early studies reporting AhR expression in the CNS of rodents detected transcriptionally active AhR including its target genes CYP1A1 and CYP1B1 in astrocytes and microglia, as well 
as in neurons of the cortex, olfactory bulb, hippocampus and cerebellum (Kainu et al. 1995; Williamson et al. 2005; Rothhammer et al. 2016; Lee et al. 2015). Depending on the ligand, it was demonstrated that AhR mediates both pro-inflammatory and anti-inflammatory effects in microglia (Lee et al. 2015).

AhR activation by agonists derived from the microbial metabolism of dietary tryptophan was discovered to limit microglia and astrocyte activities that promote CNS inflammation and neurodegeneration. The AhR is highly expressed by astrocytes and limits inflammation in the CNS through indirect, SOCS2-dependent inhibition of NF-KB and astrocytic type I interferon signaling (Rothhammer et al. 2016). In addition, AhR was reported to inhibit NF-kB activation via direct interaction with its p65 subunit (Vogel et al. 2014). Collectively, in both astrocytes and microglia, AhR acts as a negative regulator of NF-KB activation. In parallel, Rothhammer et al. could show that AhR deletion in astrocytes and microglia leads to EAE worsening and failure to recover during the disease, which was accompanied by increased production of proinflammatory mediators including $\mathrm{Ccl} 2, \mathrm{Csf} 2$ and Nos2, reflecting the heightened NF-KB activity (Rothhammer et al. 2016; Rothhammer et al. 2018; Rothhammer and Quintana 2019).

It is well-known that changes in the CNS microenvironment involve a crosstalk between astrocytes and microglia (Skripuletz et al. 2013; Rothhammer et al. 2018). After brain injury and disease, activated microglia have been reported to induce a subtype of reactive astrocytes, termed A1, by secreting tumour necrosis factor (TNF), IL-1 $\alpha$ and complement component C1q (Liddelow et al. 2017). In EAE, the AhR was found to mediate microgliaproduced TGF $\alpha$ and VEGF-B, which regulate the pathogenic activities of astrocytes and contribute to MS pathogenesis (Rothhammer 2016).

\subsection{Laquinimod (LAQ) - a novel immunomodulator}

Laquinimod (LAQ) is an oral immune-modulatory drug developed for the treatment of MS and neurodegenerative diseases. It is the successor of the compound Roquinimex (Linomide ${ }^{\circledR}$ ), which was generated by Active Biotech. Several studies on Roquinimex were stopped due to serious cardiac adverse events (Noseworthy et al. 2000). Chemical changes resulted in the discovery of LAQ showing a clearly favorable safety profile (Jonsson et al. 2004). Laquinimod, also known as Nerventra, is a quinoline-3-carboxamide derivative generated by the Swedish company Active Biotech and the Israel-based Teva Pharmaceutical Industries. The 
final product was presented as hard capsule containing $0.6 \mathrm{mg}$ of active substance Laquinimod sodium (Figure 1). It is a small molecule that passively crosses the BBB and enters the CNS (Brück and Wegner 2011). Its exact mechanism of action remains unknown, although it has been addressed in the past in numerous experimental models (Ott et al. 2019; Brück and Wegner 2011; Brück et al. 2012; Ellrichmann et al. 2017; Katsumoto et al. 2018) and in humans (Comi 2012; Vollmer et al. 2014; Giovannoni et al. 2018; Reilmann et al. 2019). On May 22 ${ }^{\text {nd }}$, 2014, marketing authorization for the medicinal product Nerventra was finally refused by the Committee for Medicinal Products for Human Use (CHMP) owing to results from animal studies indicating higher occurrence of cancers after long-term treatment and possible risks for the unborn baby (EMA 2014).

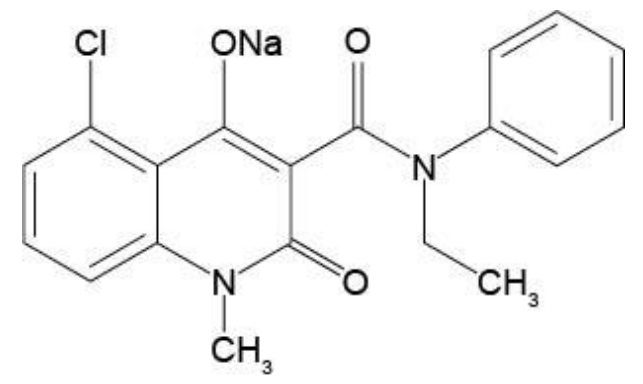

Figure 1: Structural formula of the active substance Laquinimod sodium

5-chloro-3-(ethylphenylcarbamoyl)-1-methyl-2-oxo-1,2-

dihydroquinolin-4-olate

\subsubsection{Clinical trials of LAQ}

LAQ's efficacy, safety profile and tolerability were tested in several clinical trials for the treatment of patients with relapsing-remitting multiple sclerosis (RRMS). The first phase II clinical study was conducted by Polman et al. who evaluated low dosages of $0.1-0.3 \mathrm{mg}$ LAQ per day compared to placebo. In patients with RRMS, the higher dose of $0.3 \mathrm{mg} /$ day displayed a diminished development of gadolinium-enhancing (GdE) active MRI lesions and a favorable safety profile (Polman et al. 2005). Consequent phase II trials showed that a dose of $0.6 \mathrm{mg} /$ day was also well tolerated and led to significantly decreased MRI-measured disease activity (Comi et al. 2008; Comi et al. 2010). Therefore, LAQ was further examined in three late-stage phase III studies for RRMS, namely ALLEGRO (Assessment of Oral Laquinimod in Preventing Progression in Multiple Sclerosis), BRAVO (Benefit-Risk Assessment of Avonex and Laquinimod) and CONCERTO (The Efficacy and Safety and Tolerability of Laquinimod in Subjects With Relapsing Remitting Multiple Sclerosis) (Thöne and Linker 2016). All of them were designed as randomized, double-blinded, multicenter and placebo-controlled trials to 
investigate safety, efficacy and tolerability of oral LAQ at a dosage of $0.6 \mathrm{mg} /$ day or even $1.2 \mathrm{mg}$ in the latter trial. Data from the ALLEGRO trial indicated a decelerated progression of disability and a decreased rate of relapses in patients with RRMS (Comi 2012; Brück and Vollmer 2013). Although the primary outcome measure of reducing annualized relapse rate was missed in the BRAVO trial, LAQ treatment led to more dominant effects on one of the secondary endpoints, namely brain volume change. This study revealed that $0.6 \mathrm{mg}$ LAQ per day significantly reduced brain atrophy vs. placebo and was well tolerated (Vollmer et al. 2014). Subsequently, a third phase III study of LAQ in RRMS was conducted with a higher dosage of $1.2 \mathrm{mg}$ per day. Since the primary endpoint was not reached, TEVA and Active Biotech reported discontinuity of the trial CONCERTO in 2017 (Teva Pharmaceutical Industries Ltd 2017). Later, LAQ was evaluated as a potential therapy for primary progressive MS (phase Il clinical trial "ARPEGGIO", (Giovannoni et al. 2018)), as well as for Huntington's disease (phase II clinical trial "LEGATO", (Reilmann et al. 2019)), which were both discontinued. Taken together, all clinical trials demonstrated that LAQ was generally well tolerated and did not develop any severe cardiopulmonary toxicities such as its predecessor Roquinimex. However, results from animal studies showing a higher incidence of cancers after long-term therapy and potential risks for unborn babies outweighed the beneficial effects derived from clinical studies, thus provoking the refusal of marketing authorization (EMA 2014).

\subsubsection{Experimental studies of LAQ}

Similar to the favorable outcome in clinical trials, LAQ showed pronounced effects in animal studies. The animal model most frequently used for testing novel MS treatments is the murine model of experimental autoimmune encephalomyelitis (EAE). Analyses in EAE and experimental autoimmune neuritis demonstrated that LAQ effectively prevents the progression of disease (Zou et al. 2002; Varrin-Doyer et al. 2016; Thöne et al. 2012; Wegner et al. 2010). This drug was equally protective in other models of autoimmune diseases, more specifically in lupus nephritis (Lourenco et al. 2014) and colitis (Sun et al. 2015). Studies in vivo and in vitro suggest both anti-inflammatory and neuroprotective effects.

In EAE, both preventive and therapeutic LAQ treatment diminished clinical symptoms, demyelination and CNS infiltration of macrophages and $\mathrm{T}$ cells, followed by reduced demyelination, axonal damage and astrogliosis, and increased oligodendrocyte survival 
(Moore et al. 2013; Wegner et al. 2010). Furthermore, LAQ treatment was associated with an increase in regulatory $T$ cells and a decrease in Th1 and Th17 responses. With regard to myeloid cells, LAQ triggered type II monocytes characterized by reduced production of proinflammatory IL-6 and TNF and increased anti-inflammatory IL-10 levels, thus regulating CNS autoimmunity (Schulze-Topphoff et al. 2012). This study by Schulze-Topphoff et al. demonstrates that LAQ modulates adaptive T cell immune responses via its impact on cells of the innate immune system, suggesting no direct influence on T cells.

Besides its immunomodulatory effect, EAE studies also demonstrated neuroprotective characteristics of LAQ. Mice lacking BDNF expression in both myeloid and T cells exhibited a more severe EAE disease course, indicating that LAQ might act via modulation of BDNF (Thöne et al. 2012). LAQ also exerted neuroprotective activities in EAE by preventing inflammationinduced synaptic alterations and by reducing demyelination and axonal damage (Ruffini et al. 2013; Brück and Wegner 2011).

LAQ has also been effective in the treatment of non-inflammatory CNS disease models including traumatic brain injury (Katsumoto et al. 2018), models of Huntington's disease (Ellrichmann et al. 2017; Garcia-Miralles et al. 2019) and the cuprizone mouse model (Brück et al. 2012; Kramann et al. 2016).

When a substance is available within the CNS, it can directly target CNS-intrinsic cell types. In regard to distribution in the brain, previous studies using whole-body autoradiography have shown that $7-8 \%$ of the blood concentration of LAQ crosses the BBB and enters the CNS in healthy animals, whereas $13 \%$ of LAQ reaches the brain in EAE mice (Brück and Wegner 2011). Additional evidence supporting LAQ's neuroprotective characteristics come from studies on primary CNS cells in vitro and experiments in cuprizone-induced demyelination in vivo. In the cuprizone mouse model, which is associated with an intact BBB (Torkildsen 2008), LAQ has demonstrated protective effects on oligodendrocyte precursor cell differentiation and promoted remyelination (Nyamoya et al. 2019). Moreover, LAQ was shown to prevent toxinmediated demyelination (Kramann et al. 2016), oligodendroglial apoptosis, astrocytic activation and microglial infiltration, and diminished the production of pro-inflammatory cytokines in astrocytes and microglia. While decreasing NF-kB activity in astrocytes (Brück et al. 2012), LAQ reduces the activity of several signaling pathways such as Jun- $N$-terminal kinase, ribosomal S6 kinase, and AKT/protein kinase B in activated microglia (Mishra et al. 2014). This 
indicates CNS-protective effects of LAQ by attenuating the inflammatory response in astrocytes and microglia.

\subsubsection{LAQ and AhR}

Recent work indicated that LAQ treatment elevates BDNF levels independent of AhR signaling but fails to reduce clinical symptoms, immune cell infiltration and demyelination in AhR knockout mice. This demonstrates that the immunomodulatory - but not the neuroprotective - effect of LAQ in CNS autoimmunity is mediated by the AhR (Berg et al. 2016; Kaye et al. 2016). Experiments using bone marrow chimera additionally revealed that the lack of AhR in the immune system completely overrides, while AhR deletion within the CNS partially revokes the therapeutic effect of LAQ in EAE. LAQ has been shown to attenuate EAE by inducing the generation of $\mathrm{T}_{\text {reg }}$ cells and suppression of pro-inflammatory cytokines in an AhR-dependent fashion (Kaye et al. 2016). Recent studies reported that LAQ activates natural killer (NK) cells via the AhR (Ott et al. 2019) and induces the AhR pathway in both the peripheral nervous system and the CNS of naïve and EAE mice (Birnberg et al. 2017).

Taken together, the data from our lab as well as from the literature suggest that LAQ exerts its immunomodulatory effects primarily through a direct effect on myeloid antigenpresenting cells, which leads to a modulation of T cell immune responses and attenuation of EAE severity. Until now, the exact mechanisms of action of LAQ remain unknown, in particular the molecular pathways by which LAQ mediates its therapeutic effects during cuprizoneinduced demyelination. 


\subsection{Aims of this thesis}

The AhR is highly expressed by astrocytes and has been shown to limit inflammation in the CNS (Rothhammer 2016). Previous studies in our laboratory have revealed that LAQ prevents oligodendrocyte cell death and demyelination after cuprizone ingestion through reduced astrocytic NF-KB activation (Brück et al. 2012). However, our current knowledge of the relevance of AhR signaling in glial cells is limited and the exact mechanism of action of LAQ is incompletely understood.

This thesis thus aims to answer the following questions:

a) What is the role of astrocytic and microglial AhR in CNS demyelination?

The first part of the present study evaluates the function of AhR in glial cells, in particular the impact of AhR deficiency in astrocytes and microglia on cuprizone-induced tissue damage.

b) Are the therapeutic effects of LAQ under cuprizone mediated via glial AhR signaling? The second part investigates the role of astrocytic and microglial AhR as receptors for LAQ treatment, thus elucidating the effects of astrocyte- and microglia-specific AhR deletion on LAQ efficacy during cuprizone-induced demyelination. 


\section{MATERIALS AND METHOdS}

\subsection{Materials}

\subsubsection{Reagents}

Table 1: Reagents

\begin{tabular}{|c|c|}
\hline Reagents & Source of supply \\
\hline $\begin{array}{l}\text { 4-hydroxytamoxifen, Ready Made Solution, } \\
5 \mathrm{mg} / \mathrm{ml}\end{array}$ & $\begin{array}{l}\text { Sigma-Aldrich Chemie GmbH, Steinheim, } \\
\text { Germany }\end{array}$ \\
\hline Acetic acid & Merck Millipore, Darmstadt, Germany \\
\hline Anti-Biotin MicroBeads & $\begin{array}{l}\text { Miltenyi Biotec, Bergisch Gladbach, } \\
\text { Germany }\end{array}$ \\
\hline BD Calibrite $^{\mathrm{TM}}$ & BD Biosciences, Franklin Lakes, NJ, USA \\
\hline BD FACS Shutdown Solution & BD Biosciences, Franklin Lakes, NJ, USA \\
\hline BD FACSClean ${ }^{\mathrm{TM}}$ & BD Biosciences, Franklin Lakes, NJ, USA \\
\hline BD FACSFlow $^{\mathrm{TM}}$ & BD Biosciences, Franklin Lakes, NJ, USA \\
\hline BD Perm/Wash ${ }^{\text {TM }}$ buffer & BD Biosciences, Franklin Lakes, NJ, USA \\
\hline BD Pharm lyse ${ }^{\mathrm{TM}}$ solution & BD Biosciences, Franklin Lakes, NJ, USA \\
\hline Citric acid & Merck Millipore, Darmstadt, Germany \\
\hline DAB (3,3'-Diaminobenzidine) & $\begin{array}{l}\text { Sigma-Aldrich Chemie GmbH, Steinheim, } \\
\text { Germany }\end{array}$ \\
\hline DAPI (4',6-diamidino-2-phenylindole) & $\begin{array}{l}\text { Sigma-Aldrich Chemie GmbH, Steinheim, } \\
\text { Germany }\end{array}$ \\
\hline $\begin{array}{l}\text { DMEM (High Glucose ( } 4.5 \mathrm{~g} / \mathrm{l}) \text {, with L- } \\
\text { Glutamine, without sodium pyruvate) }\end{array}$ & $\begin{array}{l}\text { Gibco, Life Technologies GmbH, Darmstadt, } \\
\text { Germany }\end{array}$ \\
\hline DMSO (dimethyl sulfoxide) & $\begin{array}{l}\text { Sigma-Aldrich Chemie GmbH, Steinheim, } \\
\text { Germany }\end{array}$ \\
\hline $\begin{array}{l}\text { dNTP (deoxyribonucleotide triphosphate) } \\
\text { mix }\end{array}$ & $\begin{array}{l}\text { Thermo Fisher Scientific, Waltham, } \\
\text { Massachusetts, USA }\end{array}$ \\
\hline $\begin{array}{l}\text { EDTA (ethylenediaminetetraacetic acid } \\
\text { disodium salt dihydrate) }\end{array}$ & $\begin{array}{l}\text { Sigma-Aldrich Chemie GmbH, Steinheim, } \\
\text { Germany }\end{array}$ \\
\hline Ethanol, absolute & Merck Millipore, Darmstadt, Germany \\
\hline ExtrAvidin-Peroxidase & $\begin{array}{l}\text { Sigma-Aldrich Chemie GmbH, Steinheim, } \\
\text { Germany }\end{array}$ \\
\hline FCS (fetal calf serum) & $\begin{array}{l}\text { Thermo Fisher Scientific, Waltham, } \\
\text { Massachusetts, USA }\end{array}$ \\
\hline FICZ (6-formylindolo[3,2-b]carbazole & $\begin{array}{l}\text { Sigma-Aldrich Chemie GmbH, Steinheim, } \\
\text { Germany }\end{array}$ \\
\hline $\begin{array}{l}\text { GelRed }^{\circledR} \text { Nucleic Acid Gel Stain, 10.000x in } \\
\text { water }\end{array}$ & Biotium, Fremont, CA, USA \\
\hline
\end{tabular}




\begin{tabular}{|c|c|}
\hline $\mathrm{H}_{2} \mathrm{O}_{2}$ (hydrogen peroxide), $30 \%$ & Merck Millipore, Darmstadt, Germany \\
\hline $\begin{array}{l}\text { HBSS (Hanks' buffered salt solution) with } \\
\mathrm{Ca}^{2+} \text { and } \mathrm{Mg}^{2+}\end{array}$ & Biochrom AG, Berlin, Germany \\
\hline $\mathrm{HCl}$ (hydrochloric acid) & Merck Millipore, Darmstadt, Germany \\
\hline HyperLadder $^{\mathrm{TM}} 50 \mathrm{bp}$ & Bioline GmbH, Luckenwalde, Germany \\
\hline IFN $\gamma$ & BioLegend, San Diego, CA, USA \\
\hline Isopropyl & Merck Millipore, Darmstadt, Germany \\
\hline Ketamine, $10 \%$ & Medistar $^{\circledR}$, Ascheberg, Germany \\
\hline Laquinimod (LAQ) & $\begin{array}{l}\text { Teva pharmaceutical industries Ltd., } \\
\text { Netanya, Israel }\end{array}$ \\
\hline Mayer's hemalum & Merck Millipore, Darmstadt, Germany \\
\hline $\mathrm{MgCl}_{2}$ (magnesium chloride), $25 \mathrm{mM}$ & $\begin{array}{l}\text { Thermo Fisher Scientific, Waltham, } \\
\text { Massachusetts, USA }\end{array}$ \\
\hline $\mathrm{NaOH}$ (sodium hydroxide solution), $1 \mathrm{M}$ & Merck Millipore, Darmstadt, Germany \\
\hline Paraffin (Paraplast Plus ${ }^{\circledR}$ ) & Tyco Healthcare, Neustadt, Germany \\
\hline Passive lysis buffer, $5 x$ & Promega, Walldorf, Germany \\
\hline PBS (phosphate buffered saline), sterile & $\begin{array}{l}\text { Gibco, Life Technologies GmbH, Darmstadt, } \\
\text { Germany }\end{array}$ \\
\hline PCR buffer, 5x Green GoTaq Reaction buffer & Promega, Walldorf, Germany \\
\hline $\begin{array}{l}\text { Penicillin-streptomycin (10000 U/ml } \\
\text { penicillin; } 10 \mathrm{mg} / \mathrm{ml} \text { streptomycin) }\end{array}$ & $\begin{array}{l}\text { Biochrom AG, Berlin, Germany; Sigma- } \\
\text { Aldrich Chemie GmbH, Steinheim, Germany }\end{array}$ \\
\hline PFA (paraformaldehyde), powder & Merck Millipore, Darmstadt, Germany \\
\hline PLL (Poly-L-Lysin hydrobromide), powder & $\begin{array}{l}\text { Sigma-Aldrich Chemie GmbH, Steinheim, } \\
\text { Germany }\end{array}$ \\
\hline Schiff's reagent & $\begin{array}{l}\text { Sigma-Aldrich Chemie GmbH, Steinheim, } \\
\text { Germany }\end{array}$ \\
\hline StarPure Low EEO Standard Agarose, $>1$ kb & Starlab GmbH, Hamburg, Germany \\
\hline Tamoxifen & $\begin{array}{l}\text { Sigma-Aldrich Chemie GmbH, Steinheim, } \\
\text { Germany }\end{array}$ \\
\hline TNF $\alpha$ & $\begin{array}{l}\text { R\&D Systems GmbH, Wiesbaden-Nordstadt, } \\
\text { Germany }\end{array}$ \\
\hline Tris (tris(hydroxymethyl)aminomethane) & $\begin{array}{l}\text { Carl Roth GmbH + Co. KG, Karlsruhe, } \\
\text { Germany }\end{array}$ \\
\hline Xylazine solution, $20 \mathrm{mg} / \mathrm{ml}$ & Ecuphar, Oostkamp, Belgium \\
\hline Xylene & $\begin{array}{l}\text { Chemsolute, Th. Geyer GmbH \& Co. KG, } \\
\text { Renningen, Germany }\end{array}$ \\
\hline
\end{tabular}




\subsubsection{Solutions, buffers and cell culture media}

Table 2: Solutions, buffers and cell culture media

\begin{tabular}{|c|c|}
\hline Solution & Composition \\
\hline $\begin{array}{l}0.1 \% \text { PLL solution for surface coating } \\
\text { (primary astrocytes) }\end{array}$ & $\begin{array}{l}1000 \mathrm{ml} \text { distilled water, sterile } \\
100 \mathrm{mg} \mathrm{PLL} \\
\text { Incubate at room temperature for at least } 30 \mathrm{~min}\end{array}$ \\
\hline Astrocyte culture medium & $\begin{array}{l}\text { DMEM } \\
10 \% \mathrm{FCS} \\
100 \mathrm{U} / \mathrm{ml} \text { penicillin } \\
0.1 \mathrm{mg} / \mathrm{ml} \text { streptomycin }\end{array}$ \\
\hline DNase $\mathrm{I}, 5 \mathrm{mg} / \mathrm{ml}$ solution & $\begin{array}{l}100 \text { mg DNase I } \\
20 \mathrm{ml} \text { HBSS, sterile }\end{array}$ \\
\hline $\begin{array}{l}\text { FACS (fluorescence-activated cell } \\
\text { sorting) buffer }\end{array}$ & $\begin{array}{l}\text { PBS } \\
2 \% \text { FCS }\end{array}$ \\
\hline LFB working solution & $\begin{array}{l}0.1 \% \text { LFB }(\mathrm{w} / \mathrm{v}) \\
0.5 \% \text { acetic acid in ethanol }\end{array}$ \\
\hline $\begin{array}{l}\text { MACS (magnetic-activated cell } \\
\text { sorting) buffer }\end{array}$ & $\begin{array}{l}\text { PBS, sterile } \\
0.5 \% \text { FCS } \\
2 \text { mM EDTA }\end{array}$ \\
\hline Paraformaldehyde (PFA), 4\% solution & $\begin{array}{l}\text { PBS } \\
4 \% \text { PFA } \\
\text { pH } 7.4\end{array}$ \\
\hline tail lysis buffer & $\begin{array}{l}0.1 \mathrm{M} \text { Tris- } \mathrm{HCl} \\
200 \mathrm{mM} \mathrm{NaCl} \\
5 \mathrm{mM} \text { EDTA } \\
0.2 \% \text { SDS } \\
\text { bidistilled water } \\
\mathrm{pH} 9.5\end{array}$ \\
\hline
\end{tabular}

Table 3: Solutions and buffers for histochemistry and immunohistochemistry

\begin{tabular}{|l|l|}
\hline Solution & Composition \\
\hline $1 \% \mathrm{HCl}$ & $1 \% \mathrm{HCl}$ absolute \\
& $70 \%$ ethanol \\
\hline Blocking buffer for & PBS \\
immunohistochemistry & $10 \%$ Goat serum \\
\hline Citric acid buffer, $10 \mathrm{mM}$ & $2.1 \mathrm{~g}$ citric acid \\
& 1 I distilled water \\
& $\mathrm{NaOH}$ \\
\hline DAB working solution & $\mathrm{pH} 6$ \\
\hline
\end{tabular}




\begin{tabular}{|l|l|}
\hline & $1 \mathrm{ml}$ DAB \\
& $20 \mu \mathrm{l}$ hydrogen peroxidase \\
\hline HCl-Isopropanol & $70 \%$ Isopropyl alcohol \\
& $0.25 \% \mathrm{HCl}$ \\
\hline LFB working solution & $1 \mathrm{~g}$ LFB \\
& Solve in $1000 \mathrm{ml} 96 \%$ Ethanol \\
& Add $5 \mathrm{ml} 10 \%$ acetic acid and filtrate solution \\
\hline TBE buffer, 10x & $108 \mathrm{~g}$ Tris \\
& $55 \mathrm{~g}$ Boric Acid \\
& $40 \mathrm{ml} 0.5 \mathrm{M}$ EDTA \\
& Fill up to $1000 \mathrm{ml}$ with distilled water \\
\hline Tris-EDTA buffer, 10x & $12.1 \mathrm{~g}$ Tris \\
& $2.92 \mathrm{~g}$ EDTA \\
& Fill up to $1000 \mathrm{ml}$ with distilled water \\
& pH 8.0 \\
\hline
\end{tabular}

\subsubsection{Antibodies, enzymes and proteins}

Table 4: Primary antibodies for immunohistochemical stainings

\begin{tabular}{|l|l|l|l|l|l|}
\hline Antigen & Marker for & $\begin{array}{l}\text { Species/ } \\
\text { Clonality/ } \\
\text { antigen }\end{array}$ & $\begin{array}{l}\text { Antigen } \\
\text { retrieval/ } \\
\text { fixation }\end{array}$ & $\begin{array}{l}\text { Working } \\
\text { dilution }\end{array}$ & $\begin{array}{l}\text { Source of } \\
\text { supply }\end{array}$ \\
\hline APP & $\begin{array}{l}\text { Amyloid } \\
\text { precursor } \\
\text { protein, early } \\
\text { axonal damage }\end{array}$ & $\begin{array}{l}\text { Mouse/ } \\
\text { monoclonal/ } \\
22 C 11\end{array}$ & $\begin{array}{l}\text { Microwave/ } \\
\text { citric acid } \\
\text { buffer }\end{array}$ & $1: 1000$ & $\begin{array}{l}\text { Merck } \\
\text { Millipore }\end{array}$ \\
\hline Mac3 & $\begin{array}{l}\text { activated } \\
\text { microglia }\end{array}$ & $\begin{array}{l}\text { Rat/ } \\
\text { polyclonal/ } \\
\text { M3/84 }\end{array}$ & $\begin{array}{l}\text { Microwave/ } \\
\text { citric acid } \\
\text { buffer }\end{array}$ & $1: 200$ & $\begin{array}{l}\text { BD } \\
\text { Pharmingen }\end{array}$ \\
\hline MBP & $\begin{array}{l}\text { Myelin protein } \\
\text { Rabbit/ } \\
\text { polyclonal/ } \\
62301\end{array}$ & $\begin{array}{l}- \\
\text { Rabbit/ } \\
\text { monoclonal/ - }\end{array}$ & $\begin{array}{l}\text { Microwave/ } \\
\text { Tris-EDTA }\end{array}$ & $1: 500$ & Abcam \\
\hline mPPP25 & $\begin{array}{l}\text { mature } \\
\text { oligodendrocytes }\end{array}$ & & Dako \\
\hline
\end{tabular}


Table 5: Secondary antibodies for immunohistochemical stainings

\begin{tabular}{|l|l|l|l|}
\hline Secondary Antibody & $\begin{array}{l}\text { Working } \\
\text { dilution }\end{array}$ & Manufacturer \\
\hline anti-mouse IgG, biotinylated & $1: 100$ & $\begin{array}{l}\text { GE Healthcare Europe GmbH, Freiburg, } \\
\text { Germany }\end{array}$ \\
\hline goat anti-rat IgG, biotinylated & $1: 200$ & Dianova GmbH, Hamburg, Germany \\
\hline $\begin{array}{l}\text { goat-anti-rabbit IgG, Biotin- } \\
\text { SP-conjugated AffiniPure }\end{array}$ & $1: 250$ & $\begin{array}{l}\text { Jackson ImmunoResearch Laboratories, Inc., } \\
\text { West Grove, PA, USA }\end{array}$ \\
\hline
\end{tabular}

Table 6: Monoclonal antibodies for flow cytometry

\begin{tabular}{|l|l|l|l|l|}
\hline Specificity & Fluorochrome & Clone & Dilution & Source of supply \\
\hline ACSA-2 & PE & IH3-18A3 & $1: 50$ & $\begin{array}{l}\text { Miltenyi Biotec, Bergisch } \\
\text { Gladbach, Germany }\end{array}$ \\
\hline CD11b & PerCP/Cy5.5 & M1/70 & $1: 100$ & BioLegend, San Diego, CA, USA \\
\hline CD16/CD32 & - & 93 & $1: 50$ & BioLegend, San Diego, CA, USA \\
\hline CD45 & APC-Cy7 & $30-$ F11 & $1: 100$ & BioLegend, San Diego, CA, USA \\
\hline GFAP & Alexa Fluor 647 & $2.2 B 10$ & $1: 100$ & $\begin{array}{l}\text { Thermo Fisher Scientific, } \\
\text { Waltham, Massachusetts, USA }\end{array}$ \\
\hline GLAST & APC & ACSA-1 & $1: 50$ & $\begin{array}{l}\text { Miltenyi Biotec, Bergisch } \\
\text { Gladbach, Germany }\end{array}$ \\
\hline unknown & APC & MOPC-173 & $1: 300$ & BioLegend, San Diego, CA, USA \\
\hline unknown & PE & RTK4530 & $1: 300$ & BioLegend, San Diego, CA, USA \\
\hline O4 & Alexa Fluor 488 & O4 & $1: 50$ & $\begin{array}{l}\text { R\&D Systems GmbH, } \\
\text { Wiesbaden-Nordstadt, } \\
\text { Germany }\end{array}$ \\
\hline
\end{tabular}

Abbreviations: $\mathbf{C D}=$ cluster of differentiation; $\mathbf{C y 5 . 5}=$ cyanine 5.5; $\mathbf{P E}=$ phycoerythrin; PerCP = peridinin chlorophyll protein; APC = allophycocyanin; APC-Cy7 = allophycocyanin cyanine 7

Table 7: Monoclonal antibody for magnetic-activated cell sorting

\begin{tabular}{|l|l|l|}
\hline Specificity & Species/Clonality/ antigen & Source of supply \\
\hline CD11b & $\begin{array}{l}\text { Rat Biotin anti-mouse/human/ } \\
\text { monoclonal/ M1/70 }\end{array}$ & $\begin{array}{l}\text { BioLegend, San Diego, CA, } \\
\text { USA }\end{array}$ \\
\hline
\end{tabular}

Table 8: Proteins and enzymes

\begin{tabular}{|l|l|}
\hline Proteins/ enzymes & Source of supply \\
\hline DNase I & Worthington, Lakewood, USA \\
\hline ExtrAvidin-Peroxidase (0.01\% solution) & $\begin{array}{l}\text { Sigma-Aldrich Chemie GmbH, Steinheim, } \\
\text { Germany }\end{array}$ \\
\hline GoTaq DNA polymerase, $500 \mathrm{U}$ & Promega, Walldorf, Germany \\
\hline
\end{tabular}




\begin{tabular}{|l|l|}
\hline Proteinase K, recombinant, PCR Grade & $\begin{array}{l}\text { Sigma-Aldrich Chemie GmbH, Steinheim, } \\
\text { Germany }\end{array}$ \\
\hline qPCRBIO Probe Mix Lo-ROX & Nippon Genetics Europe, Düren, Germany \\
\hline Trypsin-EDTA $(0.05 \% / 0.02 \%$ in PBS) & Biochrom AG, Berlin, Germany \\
\hline
\end{tabular}

\subsubsection{Applied kits}

Table 9: Applied kits

\begin{tabular}{|l|l|}
\hline Kit & Source of supply \\
\hline Cignal Lenti NF-KB Reporter Kit & Qiagen GmbH, Hilden, Germany \\
\hline Dual Luciferase ${ }^{\circledR}$ Reporter Assay System & Promega, Walldorf, Germany \\
\hline $\begin{array}{l}\text { High-Capacity cDNA Reverse Transcription } \\
\text { Kit with RNase Inhibitor }\end{array}$ & $\begin{array}{l}\text { Applied biosystems by Thermo Fisher } \\
\text { Scientific, USA }\end{array}$ \\
\hline miRNeasy Mini Kit & Qiagen GmbH, Hilden, Germany \\
\hline RNeasy Mini Kit & Qiagen GmbH, Hilden, Germany \\
\hline
\end{tabular}

\subsubsection{Oligonucleotide primers}

Table 10: Oligonucleotide primers used for transgenic mice genotyping

\begin{tabular}{|c|c|c|c|}
\hline Mouse line & Primer & Sequence $\left(5^{\prime}->3^{\prime}\right)$ & Function \\
\hline \multirow{2}{*}{$\begin{array}{l}\text { GFAP-Cre mice } \\
73.12 \mathrm{Mvs} / \mathrm{J}\end{array}$} & GFAPcre 859 & $\begin{array}{l}\text { 5'-GTG AAA CAG CAT } \\
\text { TGC TGT CAC TT-3' }\end{array}$ & $\begin{array}{l}\text { Forward primer to detect } \\
\text { Cre }\end{array}$ \\
\hline & GFAPcre 860 & $\begin{array}{l}\text { 5'-GCG GTC TGG } \\
\text { CAG TAA AAA CTA TC-3' }\end{array}$ & $\begin{array}{l}\text { Reverse primer to detect } \\
\text { Cre }\end{array}$ \\
\hline \multirow{2}{*}{$\begin{array}{l}\text { Aldh1l1- } \\
\text { CreERT2 mice }\end{array}$} & 20713 & $\begin{array}{l}\text { 5'-CAA CTC AGT CAC } \\
\text { CCT GTG CTC-3' }\end{array}$ & $\begin{array}{l}\text { Forward primer to detect } \\
\text { Aldh1l1-CreERT2 }\end{array}$ \\
\hline & 08250 & $\begin{array}{l}\text { 5'-TTC TTG CGA ACC } \\
\text { TCA TCA CTC G-3' }\end{array}$ & $\begin{array}{l}\text { Reverse primer to detect } \\
\text { Aldh1I1-CreERT2 }\end{array}$ \\
\hline \multirow{3}{*}{$\begin{array}{l}\text { CX3CR1- } \\
\text { CreERT2 mice }\end{array}$} & Common (Com) & $\begin{array}{l}\text { 5'-AAG ACT CAC GTG } \\
\text { GAC CTG CT-3' }\end{array}$ & $\begin{array}{l}\text { Common primer to detect } \\
\text { CX3CR1-CreERT2 }\end{array}$ \\
\hline & Mut & $\begin{array}{l}\text { 5'-CGG TTA TTC AAC } \\
\text { TTG CAC CA-3' }\end{array}$ & $\begin{array}{l}\text { primer to detect CX3CR1- } \\
\text { CreERT2 mutant allele }\end{array}$ \\
\hline & WT & $\begin{array}{l}\text { 5'-AGG ATG TTG ACT } \\
\text { TCC GAG TGG-3' }\end{array}$ & $\begin{array}{l}\text { primer to detect CX3CR1- } \\
\text { CreERT2 wild type allele }\end{array}$ \\
\hline \multirow{2}{*}{$A h R^{f l / f l}$ mice } & $\begin{array}{l}\text { Ahrtm3.1Bra } \\
\text { forward }\end{array}$ & $\begin{array}{l}\text { 5'-GGT ACA AGT GCA } \\
\text { CAT GCC TGC-3' }\end{array}$ & $\begin{array}{l}\text { Forward primer to detect } \\
\text { AhR exon } 2\end{array}$ \\
\hline & Ahr ${ }^{\text {tm3.1Bra-reverse }}$ & $\begin{array}{l}\text { 5'-CAG TGG GAA TAA } \\
\text { GGC AAG GA-3' }\end{array}$ & $\begin{array}{l}\text { reverse primer to detect } \\
\text { AhR exon } 2\end{array}$ \\
\hline
\end{tabular}

Source of supply: All oligonucleotide primers were obtained from Eurofins Scientific, Germany. 


\subsubsection{Primers for qPCR}

Table 11: $\operatorname{TaqMan}^{\circledR}$ qPCR primers

\begin{tabular}{|l|l|l|l|}
\hline $\begin{array}{l}\text { Gene } \\
\text { symbol }\end{array}$ & Gene name & Primer number & Source of supply \\
\hline GAPDH & $\begin{array}{l}\text { glyceraldehyde-3- } \\
\text { phosphate dehydrogenase }\end{array}$ & Mm99999915_g1 & $\begin{array}{l}\text { Life Technologies } \\
\text { GmbH, Darmstadt, } \\
\text { Germany }\end{array}$ \\
\hline B2m & beta-2 microglobulin & Mm00437762_m1 & $\begin{array}{l}\text { Life Technologies } \\
\text { GmbH, Darmstadt, } \\
\text { Germany }\end{array}$ \\
\hline CYP1A1 & $\begin{array}{l}\text { cytochrome P450 enzyme, } \\
\text { family 1, subfamily A, } \\
\text { polypeptide 1 }\end{array}$ & Mm00487217_m1 & $\begin{array}{l}\text { Life Technologies } \\
\text { GmbH, Darmstadt, } \\
\text { Germany }\end{array}$ \\
\hline
\end{tabular}

\subsubsection{Consumables}

Table 12: Consumables

\begin{tabular}{|l|l|}
\hline Consumable & Source of supply \\
\hline $40 \mu \mathrm{m}$ cell strainer & BD Biosciences, Germany \\
\hline 96-well Multiply PCR Plate Biosphere Plus & Sarstedt, Nümbrecht, Germany \\
\hline 96-well plate, optical polymer film bottom & $\begin{array}{l}\text { Thermo Fisher Scientific, Waltham, } \\
\text { Massachusetts, USA }\end{array}$ \\
\hline Cell culture flask, $25 \mathrm{~cm}^{2}$ & Greiner bio-one, Kremsmünster, Austria \\
\hline Cell culture flask, $75 \mathrm{~cm}^{2}$ & Greiner bio-one, Kremsmünster, Austria \\
\hline $\begin{array}{l}\text { Cell culture plate, Nunclon }{ }^{\text {TM }} \text { Delta } \\
\text { Surface, } 96 \text {-well U bottom }\end{array}$ & $\begin{array}{l}\text { Thermo Fisher Scientific, Waltham, } \\
\text { Massachusetts, USA }\end{array}$ \\
\hline FACS tubes, $5 \mathrm{ml}$ & BD Biosciences, Franklin Lakes, NJ, USA \\
\hline Filter tips (10 $\mu \mathrm{l}, 200 \mu \mathrm{l}, 1000 \mu \mathrm{l})$ & Nippon Genetics Europe, Düren, Germany \\
\hline LS columns & Miltenyi Biotec, Bergisch Gladbach, Germany \\
\hline Needles & BD Biosciences, Franklin Lakes, NJ, USA \\
\hline PCR strips of 8 tubes, $0.2 \mathrm{ml}$ & Sarstedt, Nümbrecht, Germany \\
\hline SD100 Cellometer counting chambers & Cenibra, Bramsche, Germany \\
\hline Syringes & BD Biosciences, Franklin Lakes, NJ, USA \\
\hline Tubes ( $50 \mathrm{ml}, 15 \mathrm{ml}, 2 \mathrm{ml}, 1.5 \mathrm{ml}, 0.5 \mathrm{ml}$, & Sarstedt, Nümbrecht, Germany \\
$0.2 \mathrm{ml})$ & \\
\hline Abbrions: & \\
\hline
\end{tabular}

Abbreviations: FACS = fluorescence-activated cell sorting 


\subsubsection{Technical devices}

Table 13: Technical devices

\begin{tabular}{|c|c|}
\hline Device & Source of supply \\
\hline Biometra thermal cycler TRIO & Analytik Jena, Germany \\
\hline $\begin{array}{l}\text { BX51 Olympus light microscope equipped } \\
\text { with DP71 digital and XM10 monochrome } \\
\text { camera }\end{array}$ & Olympus, Hamburg, Germany \\
\hline Cellometer Auto T4 & Cenibra, Bramsche, Germany \\
\hline Centrifuge $5415 \mathrm{R}$ & Eppendorf, Hamburg, Germany \\
\hline Centrifuge $5810 \mathrm{R}$ & Eppendorf, Hamburg, Germany \\
\hline LSRFortessa $^{\mathrm{TM}}$ & BD Biosciences, Franklin Lakes, NJ, USA \\
\hline Microwave NN-E201WM & Panasonic, Hamburg, Germany \\
\hline $\begin{array}{l}\text { Mithras }^{2} \text { LB } 943 \text { Multimode Microplate } \\
\text { Reader }\end{array}$ & $\begin{array}{l}\text { Berthold Technologies, Bad Wildbad, } \\
\text { Germany }\end{array}$ \\
\hline NanoDrop ND-1000 & $\begin{array}{l}\text { Peqlab, VWR International GmbH, Erlangen, } \\
\text { Germany }\end{array}$ \\
\hline Neubauer chamber & $\begin{array}{l}\text { Superior Marienfeld, Lauda-Königshofen, } \\
\text { Germany }\end{array}$ \\
\hline PowerPac 300 & BIO-Rad, Feldkirchen, Germany \\
\hline QuadroMACS ${ }^{\mathrm{TM}}$ separator & $\begin{array}{l}\text { Miltenyi Biotec, Bergisch Gladbach, } \\
\text { Germany }\end{array}$ \\
\hline QuantStudio $^{\mathrm{TM}} 7$ Flex System & $\begin{array}{l}\text { Thermo Fisher Scientific, Waltham, } \\
\text { Massachusetts, USA }\end{array}$ \\
\hline
\end{tabular}

Abbreviations: FACS = fluorescence-activated cell sorting; MACS = magnetic-activated cell sorting

\subsubsection{Software}

Table 14: Software

\begin{tabular}{|l|l|l|}
\hline Software & Application & Source of supply \\
\hline BD FACS Diva Software 8.0.2 & $\begin{array}{l}\text { Flow cytometry data } \\
\text { acquisition and analysis }\end{array}$ & $\begin{array}{l}\text { BD Biosciences, Franklin } \\
\text { Lakes, NJ, USA }\end{array}$ \\
\hline GraphPad Prism 6 & $\begin{array}{l}\text { Statistical analysis and } \\
\text { graphs }\end{array}$ & $\begin{array}{l}\text { GraphPad software Inc., La } \\
\text { Jolla, CA, USA }\end{array}$ \\
\hline
\end{tabular}

\subsection{Animals}

C57BL/6J mice and all transgenic mice used for the experiments were derived from inhouse breeding colonies at the animal facility of the University Medical Center Göttingen. 
Up to 5 animals per cage were housed together on a 12/12 h light/dark cycle at constant temperature and humidity including access to food and water ad libitum under SPF conditions. Before each experiment, animals were allowed to adapt to the new environment for at least one week. All animal experiments were carried out in accordance with the European Communities Council Directive of 24 November 1986 (86/EEC) and were admitted by the Government of Lower Saxony, Germany (17/2528).

\section{AhR-floxed mice (AhR ${ }^{\mathrm{fl} / \mathrm{fl}}$ mice)}

The $A h R^{f l / f l}$ line, also designated as $A h R^{f x}$ line, was purchased from Charles River Laboratories, Sulzfeld, Germany. AhR ${ }^{f / f l}$ mice were generated and characterized by Christopher A. Bradfield in 2005 (Walisser 2005), possess loxP sites on either side of exon 2 of the targeted gene, and are useful for generating cell-specific mutants of AhR.

\section{GFAP-specific AhR knockout mice (GFAP-Cre/+AhR ${ }^{\mathrm{fl} / \mathrm{fl}}$ mice)}

To generate mice with a conditional AhR knockout in GFAP-positive cells, the $A h R^{f l / f l}$ line was crossed with the GFAP-Cre line 73.12.

The GFAP-Cre line 73.12 was purchased from Charles River laboratories, Sulzfeld, Germany. GFAP-Cre 73.12 mice were generated and characterized by Michael V. Sofroniew in 2004 (Garcia et al. 2004). This line was backcrossed to C57BL/6 animals for at least 14 generations. Cre recombinase activity under control of the GFAP promoter targets essentially all activated astrocytes following CNS injury as well as most astrocytes during development and throughout the healthy brain and spinal cord.

\section{Inducible Aldh1|1-specific AhR knockout mice (Aldh1|1-CreERT2/+ AhR ${ }^{\mathrm{fl} / \mathrm{fl}}$ mice)}

To obtain mice with an inducible AhR knockout in Aldh1l1-expressing cells, $A_{h R^{f l / f l}}$ and Aldh1l1-CreERT2/+ animals were crossed.

The tamoxifen-inducible Aldh1/1-CreERT2/+ transgenic mouse line was generated, characterized and provided by Dr. Gesine Saher, Max Planck Institute of Experimental Medicine, Department of Neurogenetics, Göttingen (Winchenbach et al. 2016). Aldh1l1 was found to be selectively expressed in both developing and mature astrocytes (Molofsky et al. 2013; Neymeyer et al. 1997). While adult astrocytes can be targeted with high efficiency and 
specificity, Aldh1l1 shows a considerably broader pattern of expression than GFAP by labeling also cortical astrocytes in vivo (Cahoy et al. 2008; Yoon et al. 2017; Winchenbach et al. 2016; Srinivasan et al. 2016). To achieve an efficient deletion of AhR in Aldh $1 \mid 1^{+}$astrocytes, 8- to 19week-old Aldh1l1-CreERT2/+ AhR ${ }^{\mathrm{fl} / \mathrm{fl}}$ (Aldh1l1-AhR) mice and littermate controls received tamoxifen injections at a concentration of $75 \mathrm{mg}$ tamoxifen/ $\mathrm{kg}$ body weight on 5 consecutive days.

\section{Inducible CX3CR1-specific AhR knockout mice (CX3CR1-CreERT2/+ AhR ${ }^{\mathrm{fl} / \mathrm{fl}}$ mice)}

The tamoxifen-inducible CX3CR1-CreERT2/+ mouse line was purchased from Charles River Laboratories, Sulzfeld, Germany. CX3CR1-CreERT2/+ mice were generated and characterized by Dr. Dan R. Littman in 2013 (Littman 2013; Parkhurst et al. 2013). This line expresses a Cre-ERT2 fusion protein and an enhanced yellow fluorescent protein (EYFP) from endogenous CX3CR1 promoter/enhancer elements. CX3CR1-expressing microglia in the brain can be observed by EYFP fluorescence.

To generate CX3CR1 cell-specific AhR conditional knockout mice, CX3CR1CreERT2/+ AhR ${ }^{\mathrm{fl} / \mathrm{fl}}$ mice, expressing the Cre recombinase under control of the CX3CR1 regulatory sequence (promoter), were crossed with $\mathrm{AhR}^{\mathrm{fl} / \mathrm{fl}}$ mice. Adult CX3CR1CreERT2/+AhR ${ }^{\mathrm{fl} / \mathrm{fl}}$ (CX3CR1-AhR) mice and littermate controls at the age of 9 to 16 weeks were treated with tamoxifen at a concentration of $75 \mathrm{mg}$ tamoxifen/kg body weight on 5 consecutive days to accomplish Cre-mediated recombination.

\subsection{Methods}

\subsubsection{Animal experiments}

\subsubsection{Genotyping}

Genotyping of genetically modified mice was performed in cooperation with Ms. Katja Schulz (Department of Neuropathology, University Medical Center Göttingen).

Genomic DNA was extracted from tail biopsies of GFAP-Cre/+ AhR ${ }^{\mathrm{fl} / \mathrm{fl}}$ mice, Aldh1l1CreERT2/+ AhR $R^{f l / f l}$ mice and CX3CR1-CreERT2/+ AhR ${ }^{\mathrm{fl} / f 1}$ mice. Tail biopsies were digested in $350 \mu \mathrm{l}$ tail lysis buffer with $20 \mu \mathrm{l}$ of proteinase $\mathrm{K}$ for 3 hours or overnight ( $350 \mathrm{rpm}, 56^{\circ} \mathrm{C}$ ). To remove remaining tissue, the samples were centrifuged for $10 \mathrm{~min}$ at 13,200 rpm and room 
temperature. The genomic DNA in the supernatant was transferred to a new tube, precipitated with $350 \mu \mathrm{l}$ isopropyl alcohol and centrifuged for $10 \mathrm{~min}$ at 13,200 rpm and room temperature. The supernatant was discarded and the pellet was washed with $350 \mu \mathrm{l} 70 \%$ ethanol. After centrifugation for $10 \mathrm{~min}$ at $13,200 \mathrm{rpm}$ and room temperature, the supernatant was discarded again and the pellet was dried in a speed vacuum concentrator for $10 \mathrm{~min}$. The dried pellet was resuspended in $100 \mu$ l bidistilled water and stored at $4^{\circ} \mathrm{C}$.

\section{Genotyping of GFAP-Cre/+}

Each PCR reaction contained:

Table 15: PCR reaction mix for GFAP-Cre/+

\begin{tabular}{|l|l|}
\hline Volume & Component \\
\hline $2 \mu \mathrm{l}$ & genomic DNA \\
\hline $2.5 \mu \mathrm{l}$ & $5 x$ Go-Taq DNA polymerase buffer \\
\hline $0.15 \mu \mathrm{l}$ & $10 \mathrm{mM}$ dNTP mix \\
\hline $0.25 \mu \mathrm{l}$ & 100 pmol oligonucleotide primer (859) \\
\hline $0.25 \mu \mathrm{l}$ & 100 pmol oligonucleotide primer (860) \\
\hline $0.2 \mu \mathrm{l}$ & PfuS DNA polymerase* \\
\hline $6.5 \mu \mathrm{l}$ & bidistilled water \\
\hline $0.8 \mu \mathrm{l}$ & $25 \mathrm{mM} \mathrm{MgCl}_{2}$ \\
\hline
\end{tabular}

*PfuS DNA polymerase was manufactured by Dr. Franziska van der Meer (Department of Neuropathology, University Medical Center Göttingen).

PCR reactions were run in a Biometra thermocycler (Biometra TRIO) at the following cycling conditions:

Table 16: PCR cycling conditions for GFAP-Cre/+

\begin{tabular}{|l|l|l|}
\hline Temperature & Time & Step \\
\hline $98.5^{\circ} \mathrm{C}$ & $3 \mathrm{~min}$ & Initial denaturation \\
\hline $98.5^{\circ} \mathrm{C}$ & $30 \mathrm{~s}$ & Denaturation \\
\hline $65^{\circ} \mathrm{C}$ & $45 \mathrm{~s}$ & Annealing \\
\hline $72^{\circ} \mathrm{C}$ & $1 \mathrm{~min}$ & \\
\hline $72^{\circ} \mathrm{C}$ & $2 \mathrm{~min}$ & Extension \\
\hline $4^{\circ} \mathrm{C}$ & $\infty$ & Final extension \\
\hline
\end{tabular}

To analyze the amplified PCR product, the samples $(16 \mu \mathrm{l})$ were loaded on a $3 \%$ agarose gel in 1x TBE buffer containing GelRed. Electrophoresis was performed at $130 \mathrm{~V}$ for $45-90 \mathrm{~min}$. PCR 
product length was evaluated in relation to a $50 \mathrm{bp}$ DNA ladder. A band at $100 \mathrm{bp}$ was characteristic for the Cre recombinase allele.

\section{Genotyping of Aldh1l1-CreERT2/+}

Each PCR reaction contained:

Table 17: PCR reaction mix for Aldh1l1-CreERT2/+

\begin{tabular}{|l|l|}
\hline Volume & Component \\
\hline $2 \mu \mathrm{l}$ & genomic DNA \\
\hline $4 \mu \mathrm{l}$ & $5 x$ PCR buffer \\
\hline $1 \mu \mathrm{l}$ & 2,5 mM dNTP mix \\
\hline $0.5 \mu \mathrm{l}$ & 10 pmol oligonucleotide primer (20713) \\
\hline $0.5 \mu \mathrm{l}$ & 10 pmol oligonucleotide primer (8250) \\
\hline $0.1 \mu \mathrm{l}$ & GoTaq polymerase \\
\hline $8.9 \mu \mathrm{l}$ & bidistilled water \\
\hline
\end{tabular}

PCR reactions were run at the following cycling conditions:

Table 18: PCR cycling conditions for Aldh1l1-CreERT2/+

\begin{tabular}{|c|c|c|c|}
\hline Temperature & Time & & Step \\
\hline $94^{\circ} \mathrm{C}$ & $3 \min$ & & Initial denaturation \\
\hline $58^{\circ} \mathrm{C}$ & $30 \mathrm{~s}$ & \multirow{3}{*}{36 cycles } & Denaturation \\
\hline $72^{\circ} \mathrm{C}$ & $1 \mathrm{~min}$ & & Annealing \\
\hline $94^{\circ} \mathrm{C}$ & $30 \mathrm{~s}$ & & Extension \\
\hline $58^{\circ} \mathrm{C}$ & \multicolumn{2}{|l|}{$1 \mathrm{~min}$} & Final extension \\
\hline $72^{\circ} \mathrm{C}$ & \multicolumn{2}{|l|}{$10 \mathrm{~min}$} & \\
\hline $4^{\circ} \mathrm{C}$ & \multicolumn{2}{|l|}{$\infty$} & Storage \\
\hline
\end{tabular}

To analyze the amplified PCR product, the samples (16 $\mu \mathrm{l})$ were loaded on a 3\% agarose gel in 1x TBE buffer containing GelRed. Electrophoresis was performed at $130 \mathrm{~V}$ for $45-90 \mathrm{~min}$. PCR product length was evaluated in relation to a $50 \mathrm{bp}$ DNA ladder. A band at $590 \mathrm{bp}$ was characteristic for the Cre recombinase allele. 


\section{Genotyping of CX3CR1-CreERT2/+}

Each PCR reaction contained:

Table 19: PCR reaction mix for CX3CR1-CreERT2/+

\begin{tabular}{|l|l|}
\hline Volume & Component \\
\hline $2 \mu \mathrm{l}$ & genomic DNA \\
\hline $4 \mu \mathrm{l}$ & $5 x$ PCR buffer \\
\hline $0.3 \mu \mathrm{l}$ & $2,5 \mathrm{mM}$ dNTP mix \\
\hline $0.5 \mu \mathrm{l}$ & 10 pmol oligonucleotide primer (WT/Mutant) \\
\hline $0.5 \mu \mathrm{l}$ & 10 pmol oligonucleotide primer (Com) \\
\hline $0.1 \mu \mathrm{l}$ & GoTaq polymerase \\
\hline $0.5 \mu \mathrm{l}$ & $25 \mathrm{mM} \mathrm{MgCl}_{2}$ \\
\hline $9.1 \mu \mathrm{l}$ & bidistilled water \\
\hline
\end{tabular}

PCR reactions were run at the following cycling conditions:

Table 20: PCR cycling conditions for CX3CR1-CreERT2/+

\begin{tabular}{|l|l|l|}
\hline Temperature & Time & Step \\
\hline $94^{\circ} \mathrm{C}$ & $3 \mathrm{~min}$ & Initial denaturation \\
\hline $94^{\circ} \mathrm{C}$ & $30 \mathrm{~S}$ & Denaturation \\
\hline $58^{\circ} \mathrm{C}$ & $45 \mathrm{~s}$ & Annealing \\
\hline $72^{\circ} \mathrm{C}$ & $45 \mathrm{~s}$ & \\
\cline { 2 - 3 } $72^{\circ} \mathrm{C}$ & $5 \mathrm{~min}$ & Extension \\
\hline $4^{\circ} \mathrm{C}$ & $\infty$ & Final extension \\
\hline
\end{tabular}

To analyze the amplified PCR product, the samples (16 $\mu \mathrm{l})$ were loaded on a 3\% agarose gel in 1x TBE buffer containing GelRed. Electrophoresis was performed at $130 \mathrm{~V}$ for 45 - $90 \mathrm{~min}$. PCR product length was evaluated in relation to a $50 \mathrm{bp}$ DNA ladder. A band at $300 \mathrm{bp}$ was characteristic for the Cre recombinase allele, while a band at 695 bp was detected for the wild type allele. Heterozygous mice displayed both bands. 


\section{Genotyping of $A \mathrm{hR}^{\mathrm{fl} / \mathrm{fl}}$}

Each PCR reaction included:

Table 21: PCR reaction mix for $A h R^{\mathrm{fl} / \mathrm{fl}}$

\begin{tabular}{|l|l|}
\hline Volume & Component \\
\hline $2 \mu \mathrm{l}$ & genomic DNA \\
\hline $4 \mu \mathrm{l}$ & $5 x$ PCR buffer \\
\hline $0.5 \mu \mathrm{l}$ & $2,5 \mathrm{mM}$ dNTP mix \\
\hline $0.5 \mu \mathrm{l}$ & $10 \mathrm{mM}$ oligonucleotide primer (For) \\
\hline $0.5 \mu \mathrm{l}$ & $10 \mathrm{mM}$ oligonucleotide primer (Rev) \\
\hline $0.1 \mu \mathrm{l}$ & GoTaq polymerase \\
\hline $9.4 \mu \mathrm{l}$ & bidistilled water \\
\hline
\end{tabular}

PCR reactions were run at the following cycling conditions:

Table 22: PCR cycling conditions for $A h R^{\mathrm{fl} / \mathrm{fl}}$

\begin{tabular}{|c|c|c|c|}
\hline Temperature & Time & & Step \\
\hline $94^{\circ} \mathrm{C}$ & $3 \mathrm{~min}$ & & Initial denaturation \\
\hline $94^{\circ} \mathrm{C}$ & $30 \mathrm{~s}$ & \multirow{3}{*}{35 cycles } & Denaturation \\
\hline $60^{\circ} \mathrm{C}$ & $20 \mathrm{~s}$ & & Annealing \\
\hline $72^{\circ} \mathrm{C}$ & $20 \mathrm{~s}$ & & Extension \\
\hline $72^{\circ} \mathrm{C}$ & \multicolumn{2}{|c|}{$2 \min$} & Final extension \\
\hline $4^{\circ} \mathrm{C}$ & \multicolumn{2}{|l|}{$\infty$} & Storage \\
\hline
\end{tabular}

To analyze the amplified PCR product, the samples $(16 \mu \mathrm{l})$ were loaded on an agarose gel in 1x TBE buffer containing GelRed. The endogenous allele was characterized by a band at $106 \mathrm{bp}$, while the floxed allele was detected by a band at $140 \mathrm{bp}$. Heterozygous animals displayed both bands.

\subsubsection{Cuprizone experiments}

Cuprizone is a copper chelator, which induces oligodendrocyte apoptosis and subsequent demyelination, microglial activation and astrogliosis. In this experimental animal model, mice are fed with this substance, resulting in toxic demyelination of the corpus callosum, cortex and cerebellum, while the blood-brain barrier (BBB) remains intact. It is commonly used to 
study the molecular mechanisms involved in CNS de- and remyelination in the near absence of peripheral immune cell infiltration (Hiremath et al. 1998).

\subsection{Tamoxifen preparation and treatment}

Tamoxifen was purchased from Sigma-Aldrich, Steinheim, Germany. The compound was dissolved in corn oil at a concentration of $7.5 \mathrm{mg} / \mathrm{ml}$ by rotating for 2 hours at $37^{\circ} \mathrm{C}$. The lightsensitive solution was kept in the dark and stored at $4^{\circ} \mathrm{C}$. Tamoxifen was administered via intraperitoneal injection at a concentration of $75 \mathrm{mg}$ tamoxifen/ $\mathrm{kg}$ body weight.

\subsection{LAQ preparation and treatment}

Laquinimod (LAQ) was kindly provided by Teva Pharmaceutical Industries, Ltd., Netanya, Israel. The compound was dissolved in tap water and stored at $4^{\circ} \mathrm{C}$. Mice received daily treatment with LAQ at a dosage of $25 \mathrm{mg} / \mathrm{kg}$ body weight by oral gavage. As control treatment, mice received the same volume of tap water.

\subsection{Time schedule and experimental setup}

8- to 24-week-old GFAP-Cre/+ AhR ${ }^{\mathrm{fl} / \mathrm{fl}}$ (Figure $2 \mathrm{~A}$ ), Aldh1l1-CreERT2/+AhR ${ }^{\mathrm{fl} / \mathrm{fl}}$ and CX3CR1CreERT2/+ AhR ${ }^{\mathrm{fl} / f l}$ mice (Figure $2 \mathrm{~B}$ ), including control mice (Cre-negative littermates), were used to investigate cuprizone-induced pathology. Where required, AhR deletion in Aldh1l1 ${ }^{+}$ astrocytes or CX3CR1 ${ }^{+}$microglia was induced by injection of $75 \mathrm{mg}$ tamoxifen/ $\mathrm{kg}$ body weight on 5 consecutive days, while control mice received the same treatment. Subsequently, mice were fed with a chow containing $0.25 \%$ cuprizone ad libitum for 6 weeks. To evaluate the effect of LAQ treatment on cuprizone-induced demyelination in astrocyte- and microgliaspecific AhR-deleted mice, animals were treated with $25 \mathrm{mg} / \mathrm{kg} \mathrm{LAQ}$ or vehicle in parallel once daily by oral gavage. After 6 weeks of cuprizone treatment, mice were sacrificed and brain, spleen and liver were collected for histological and immunohistochemical analyses (see section 2.3.2). 

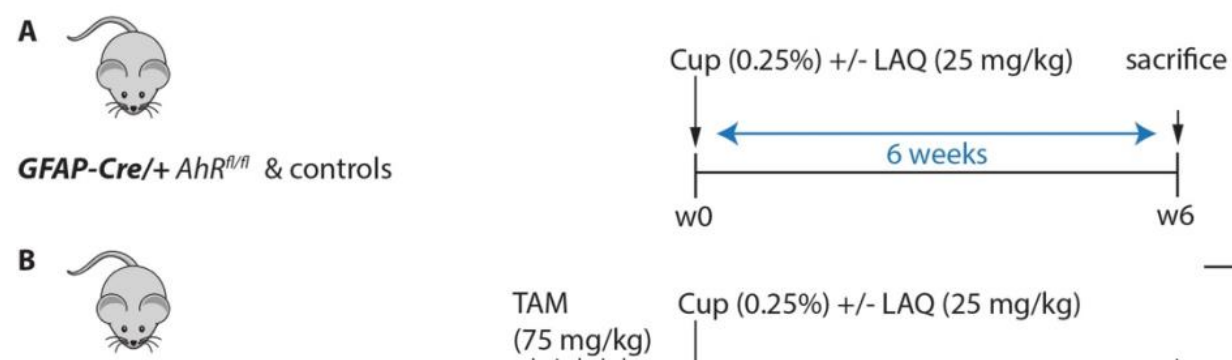

Aldh1/1-CreERT2/+AhR $R^{f / f}$ CX3CR1-CreERT2/+AhR $R^{\text {fl/ }}$

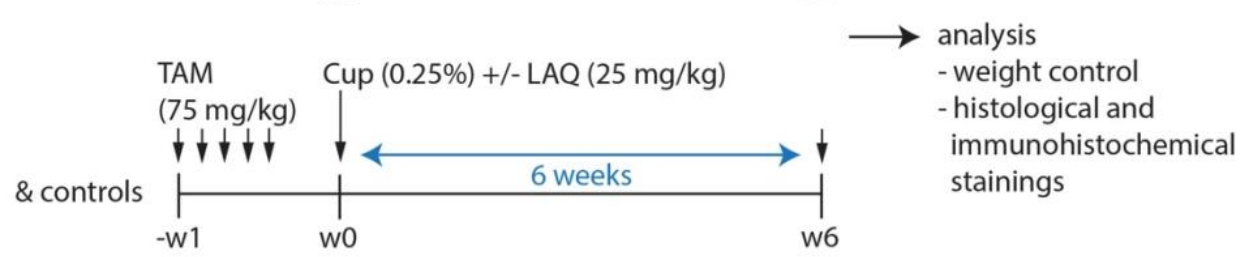

Figure 2: Time schedule of cuprizone experiments

\subsubsection{Perfusion of animals}

In order to preserve the tissue morphology for histological analyses, mice were perfused transcardially. Animals were anesthetized with an overdose of ketamine $(240 \mathrm{mg} / \mathrm{kg}$ body weight) and xylazine (40 mg/kg body weight) by intraperitoneal injection. Once the animal had reached a surgical plane of anesthesia and had lost the corneal and pedal pain reflexes, the thorax was opened. The perfusion was carried out via the left heart ventricle and simultaneous opening of the right atrium. Animals were perfused with PBS to remove the blood from the vasculature, which was followed by 4\% PFA. For histological evaluations, brain, spleen and liver were collected and post-fixed in $4 \%$ PFA for $48 \mathrm{~h}$ at $4^{\circ} \mathrm{C}$. After 2 days, brains were washed with PBS and dissected into 2-3 mm thick coronal sections. Spleen and liver were processed the same way and served as control tissue in the same paraffin block. For paraffin embedding, the tissues were gradually dehydrated via alcohol and xylene and finally embedded in paraffin using the automated tissue processor TP 1020 (Leica) overnight.

\subsubsection{Histology}

\subsubsection{Cutting, deparaffinization and hydration of paraffin-embedded sections}

For histological analysis, paraffin blocks were cut into 1-2 $\mu \mathrm{m}$ thick sections using a sliding microtome, and the coronal brain sections were mounted onto glass slides. Prior to the staining procedure, sections were deparaffinized and rehydrated. For this purpose, paraffin 
was melted by incubating the sections for at least 1 hour at $56^{\circ} \mathrm{C}$ and then transferred to xylene. The deparaffinization and rehydration steps were conducted as follows:

Table 23: Deparaffinization and rehydration steps

\begin{tabular}{|l|l|l|}
\hline Repetitions & Duration & Reagent \\
\hline $4 x$ & $10 \mathrm{~min}$ & $x y l e n e$ \\
\hline $1 x$ & $5 \mathrm{~min}$ & $x y l e n e /$ isopropyl alcohol \\
\hline $2 \mathrm{x}$ & $5 \mathrm{~min}$ & $100 \%$ isopropyl alcohol \\
\hline $1 \mathrm{x}$ & $5 \mathrm{~min}$ & $90 \%$ isopropyl alcohol \\
\hline $1 \mathrm{x}$ & $5 \mathrm{~min}$ & $70 \%$ isopropyl alcohol \\
\hline $1 \mathrm{x}$ & $5 \mathrm{~min}$ & $50 \%$ isopropyl alcohol \\
\hline & & distilled water \\
\hline
\end{tabular}

\subsubsection{Histological stainings}

\subsection{Luxol Fast Blue - Periodic Acid Schiff (LFB-PAS) staining}

LFB-PAS staining was performed to visualize myelin and determine the extent of demyelination in GFAP-, Aldh1l1- and CX3CR1-AhR conditional knockout mice treated with cuprizone. For the LFB staining, brain sections were deparaffinized, washed up to the $90 \%$ isopropyl alcohol step (see Table 23) and incubated in LFB working solution overnight at $60^{\circ} \mathrm{C}$. The following day, sections were incubated in 90\% isopropyl alcohol. For the differentiation step, sections were shortly incubated in $0.05 \%$ lithium carbonate, followed by $70 \%$ isopropyl alcohol and finally washed in distilled water to stop the differentiation. These 3 steps were repeated until only the myelin appeared deep blue. For the PAS staining, sections were incubated in 1\% periodic acid for $5 \mathrm{~min}$, washed in tap water for $5 \mathrm{~min}$ followed by distilled water and incubated in Schiff's reagent for $20 \mathrm{~min}$. Thereafter, the sections were washed with tap water for $5 \mathrm{~min}$. To counterstain the nuclei, sections were incubated in Mayer's hemalum solution for $2 \mathrm{~min}$, washed with distilled water and shortly incubated in $1 \% \mathrm{HCl}$-isopropyl alcohol for differentiation. Sections were washed with tap water and subsequently dehydrated by performing all steps listed in Table 23 in reverse order. Finally, sections were mounted in DePex mounting medium. 


\subsubsection{Immunohistochemical stainings}

Immunohistochemical stainings were performed to investigate microglial activation (Mac3), axonal damage (APP), oligodendrocyte densities (p25/TPPP) and myelination (myelin basic protein; MBP) using the labeled streptavidin-biotin method. As PFA fixation of tissues can result in masked antigens in the sections, which in turn prevents binding of the primary antibodies to these epitopes, antigens had to be unmasked. Therefore, we used two different heat-induced methods adjusted for every primary antibody (see Table 4). For retrieval of antigens, sections were heated 5 times for 3 minutes in a microwave with either $10 \mathrm{mM}$ citric acid buffer, $\mathrm{pH}$ 6.0, or 1x Tris-EDTA buffer, pH 8.0. The cooled sections were then washed with bidistilled water followed by PBS and incubated in $3 \% \mathrm{H}_{2} \mathrm{O}_{2}$ in PBS for 10 minutes at room temperature to block the endogenous peroxidase. After three washing steps with PBS, sections were incubated with $10 \%$ goat serum in PBS for 10 minutes to prevent unspecific antibody binding. Thereafter, primary antibodies diluted in blocking buffer (see Table 4) were added to the sections and incubated at $4^{\circ} \mathrm{C}$ overnight. The next day, sections were washed 3 times with PBS and incubated for 1 hour at room temperature with the corresponding biotinconjugated secondary antibodies diluted in blocking buffer (see Table 5). After incubation, sections were washed with PBS to remove unbound antibodies and incubated with $0.1 \%$ ExtrAvidin-peroxidase in blocking buffer for 1 hour at room temperature. To visualize antibody binding, slides were washed 3 times with PBS and developed in a 3,3'-diaminobenzidine (DAB) solution. The chromogen DAB is oxidized by the peroxidase, which leads to a dark brown staining. The reaction was controlled under a microscope and stopped via rinsing in distilled water. Subsequently, nuclei were counterstained with Mayer's hemalum solution for 30 seconds, the sections were washed with distilled water and blued in tap water for 10 minutes. Finally, slides were dehydrated (see Table 23, reverse order) and mounted in DePex as described above.

\subsubsection{Morphometry and data acquisition}

\subsubsection{LFB-PAS-stained sections and myelin protein MBP}

To evaluate the extent of demyelination in the corpus callosum of GFAP-, Aldh1l1- and CX3CR1-AhR conditional knockout mice after 6 weeks of cuprizone treatment, slides were 
evaluated at a magnification of $400 x$ and a semi quantitative score, as follows, was used according to Hiremath et al. (Hiremath et al. 1998):

$\begin{array}{ll}\text { Score } & \% \text { of demyelinated corpus callosum } \\ 0 & 0 \% \\ 1 & <33 \% \\ 2 & 33 \%-66 \% \\ 3 & >66 \%\end{array}$

\author{
Extent of demyelination \\ no demyelination \\ slight demyelination \\ moderate demyelination \\ strong demyelination
}

\subsubsection{Mac3-positive microglia, APP-positive spheroids and p25-positive oligodendrocytes}

The number and density of Mac3-positive cells, APP-positive spheroids and p25-positive mature oligodendrocytes were determined within the whole corpus callosum using a light microscope with an ocular morphometric grid. Positive cells were counted at $400 x$ magnification.

\subsubsection{Cell culture}

\subsubsection{Isolation of astrocytes and microglia from newborn mice}

For the isolation of astrocytes and microglia cells, newborn GFAP-AhR and Aldh1l1-AhR mice, littermate control mice and wild type C57BL6/J mice were used between postnatal day 0 (P0) and day 2 (P2). They were obtained from the in-house breeding facility.

Animals were decapitated and the skulls were immediately opened. The brains were removed and transferred into a petri dish containing cooled Hanks' buffered salt solution (HBSS buffer). In order to prevent fibroblast interference with glial cell growth, meninges were removed with forceps. Each brain was washed with HBSS and digested in $100 \mu \mathrm{l} 0.05 \%$ TrypsinEDTA solution for $10 \mathrm{~min}$ at $37^{\circ} \mathrm{C}$. During this step, brains were vortexed carefully a few times. The reaction was stopped by adding $5 \mathrm{ml}$ culture medium and the tissue was further dissociated by adding $40 \mu \mathrm{l}$ DNase and thorough pipetting. Subsequently, the cell suspension was centrifuged for $10 \mathrm{~min}$ at $200 \mathrm{~g}$ to remove debris, the pellet was resuspended at a density of 1-2 brains/ $15 \mathrm{ml}$ culture medium, seeded in PLL-coated $75 \mathrm{~cm}^{2}$ flasks and placed in an incubator with $5 \% \mathrm{CO}_{2}$ and $37^{\circ} \mathrm{C}$. The next two consecutive days, the primary cell culture was 
washed 3 times with PBS and medium was exchanged. Thereafter, the mixed glial cells were grown for 10-14 days with medium changes every 3-4 days. When the culture reached $100 \%$ confluency, the loosely sitting microglia cells and oligodendrocytes were removed by washing the cells several times with PBS, while astrocytes stayed firmly attached on the bottom of the flask. To detach the astrocyte cell layer, cells were covered with $0.05 \%$ Trypsin-EDTA solution for $3 \mathrm{~min}$ at $37^{\circ} \mathrm{C} / 5 \% \mathrm{CO}_{2}$. The reaction was stopped by adding double volume of culture medium and astrocytes were detached by vigorous shaking. After centrifugation for $10 \mathrm{~min}$ at $200 \times \mathrm{g}$, the supernatant was discarded and the pellet was resuspended in culture medium. Astrocyte concentration was determined using a Neubauer and a SD100 Cellometer counting chamber.

\subsubsection{NF-KB reporter assay}

To determine the activity of the NF-KB signaling pathway in primary cultures of astrocytes, cells were prepared from brains of newborn GFAP-AhR mice as described in section 2.3.4.1. Astrocytes from GFAP-Cre-negative littermates served as controls. Cells were seeded in triplicates in 96-well plates at a concentration of 100,000 cells per well. After $48 \mathrm{~h}$, astrocytes were transduced with an inducible NF-KB-responsive firefly luciferase reporter and a renilla reniformis luciferase normalization reporter diluted in DMEM/10\% FCS using lentiviral vectors. $24 \mathrm{~h}$ after transduction with reporter constructs, cells were washed with PBS and culture medium was exchanged. The following day, astrocytes were pre-incubated with 0 or $250 \mathrm{nM}$ LAQ for $2 \mathrm{~h}$ and subsequently stimulated with $10 \mathrm{ng} / \mathrm{ml} \mathrm{IL-1 \beta}$ and $10 \mathrm{ng} / \mathrm{ml} \mathrm{IFNy}$ for $1 \mathrm{~h}$. The reaction was stopped by washing with PBS. Cells were lysed in 1x passive lysis buffer and frozen at $-20^{\circ} \mathrm{C}$. NF-KB reporter gene expression was measured using the protocol of DualLuciferase ${ }^{\circledR}$ Reporter Assay System (Promega) according to the manufacturer's instructions.

\subsubsection{Quantitative PCR analysis of PO astrocytes and microglia}

In order to confirm cell-specific deletion of AhR or microglial responses upon stimulation with the AhR ligand FICZ, expression levels of the AhR target gene CYP1A1 were measured by qPCR. Glia cells were isolated from the brains of newborn GFAP-Cre/+AhR ${ }^{f / f l}$, Aldh1l1-

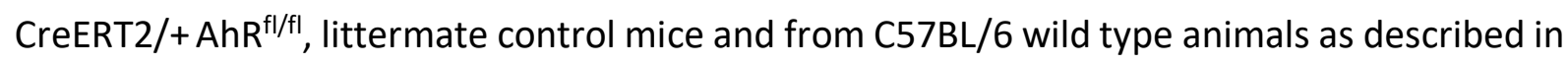
section 2.3.4.1. 


\subsubsection{Purification of astrocytes and microglia}

To obtain a pure astrocyte population, cells were purified by deletion of non-target cells (negative selection) using magnetic-activated cell sorting (MACS). For this purpose, loosely sitting microglia cells and oligodendrocytes were first removed from the astrocyte layer by shaking the flasks vigorously at $37^{\circ} \mathrm{C}$ for $1 \mathrm{~h}$. Thereafter, $0.05 \%$ Trypsin-EDTA was added to the remaining astrocyte layer for $3 \mathrm{~min}$, the reaction was stopped by adding culture medium and cells were detached by intense shaking. To avoid clumps, cells were flushed through a $40 \mu \mathrm{m}$ cell strainer and centrifuged at $200 \mathrm{~g}$ for $10 \mathrm{~min}$. The pellets were resuspended in cold MACS buffer and the cell concentration was determined using a Neubauer counting chamber. Cells were washed two additional times with MACS buffer, centrifuged and then supernatants were discarded. Cell pellets were resuspended in $20 \mu \mathrm{l}$ MACS buffer per $10^{6}$ cells and $10 \mu \mathrm{l}$ biotinylated anti-mouse/human CD11b antibody was added to label all CD11b+ microglia. After 15 min incubation on ice, unbound antibodies were removed by washing with $20 \mathrm{ml}$ MACS buffer. Cells were centrifuged, supernatants were discarded and cell pellets were resuspended in $90 \mu \mathrm{l}$ MACS buffer. $30 \mu \mathrm{l}$ anti-Biotin MicroBeads were added and incubated for $15 \mathrm{~min}$ on ice. Thereafter, MACS buffer was added to remove unbound MicroBeads and cells were centrifuged. Supernatants were discarded and cell pellets were resuspended in $1 \mathrm{ml}$ MACS buffer. Cell suspensions were placed on equilibrated LS columns and the flowthrough, which contains the unlabeled fraction of astrocytes, was collected. To obtain the labeled fraction of microglia, columns were removed from the magnetic separator, placed on a new collection tube and cells were flushed out with $5 \mathrm{ml}$ MACS buffer.

\subsubsection{Analysis of astrocyte purification by flow cytometry}

The purity of astrocytes after MACS separation was analyzed by flow cytometry. 100,000 MACS-purified cells per well were spun down in 96-well U plates. Unspecific staining was blocked by anti-CD16/CD32 diluted 1:50 in FACS buffer and incubated for 10 min at $4^{\circ} \mathrm{C}$ in the dark. Thereafter, antibodies targeting extracellular antigens were added at a final concentration of $1: 200$ and cells were incubated for 20 min at $4^{\circ} \mathrm{C}$, followed by centrifugation at $300 \mathrm{~g}$ for $5 \mathrm{~min}$ and $4^{\circ} \mathrm{C}$. Unbound antibodies were removed by washing twice with $100 \mu \mathrm{l}$ FACS buffer and the cell pellet was thereafter resuspended in $100 \mu \mathrm{l} \mathrm{BD}$ Cytofix $^{\mathrm{TM}}$ solution for $45 \mathrm{~min}$ at $4^{\circ} \mathrm{C}$ for fixation. The cells were washed twice with Perm/Wash ${ }^{\mathrm{TM}}$ buffer (10x BD 
Perm/Wash buffer, diluted $1: 10$ in distilled $\mathrm{H}_{2} \mathrm{O}$ ) and incubated for 25 minutes at room temperature in the dark with anti-GFAP antibodies (1:100 diluted in $100 \mu \mathrm{l} \mathrm{Perm} / \mathrm{Wash}^{\mathrm{TM}}$ buffer). The cells were washed twice with FACS buffer and analyzed immediately by flow cytometry (BD LSRFortessa) in cooperation with Dr. Stefan Nessler (Department of Neuropathology, University Medical Center Göttingen).

\subsubsection{CYP1A1 expression analysis}

\subsection{Evaluation of astrocyte-specific AhR deletion by FICZ treatment in vitro}

To confirm cell-specific deletion of AhR, purified P0 astrocytes from GFAP-Cre/+ AhR ${ }^{\mathrm{fl} / f \mathrm{l}}$, Aldh1/1-CreERT2/+ AhR ${ }^{\mathrm{fl} / f l}$ and respective littermate control mice as described in section 2.3.5.1 were seeded in 96 -well plates at a density of 100,000 cells per well in culture medium containing 10\% FCS and 1\% Pen/Strep. Astrocytes were cultured for $24 \mathrm{~h}$ prior to further treatments.

To induce conditional AhR deletion in Aldh1l1-positive astrocytes, cells from Aldh1l1CreERT2/+ AhR $\mathrm{R}^{\mathrm{fl} / \mathrm{fl}}$ mice and controls were then pre-incubated with $20 \mu \mathrm{M}$ 4-hydroxytamoxifen for $24 \mathrm{~h}$.

Astrocytes were treated with the AhR-specific ligand FICZ at a concentration of $100 \mathrm{nM}$ and 1\% DMSO as control for $6 \mathrm{~h}$. After FICZ stimulation, cells were lysed in RLT lysis buffer. Subsequently, RNA was isolated and transcribed into CDNA followed by quantitative PCR.

\subsection{Activation of AhR transcriptional activity by FICZ or LAQ treatment in vitro}

For measuring glial responses to FICZ stimulation, purified PO microglia or astrocytes from C57BL/6 mice as described in section 2.3.5.1 were seeded in 96-well plates at a density of 100,000 cells per well in culture medium containing 10\% FCS and 1\% Pen/Strep.

The following day, murine primary astrocytes were additionally treated with either $125 \mathrm{nM}$, $250 \mathrm{nM}$ or $500 \mathrm{nM}$ LAQ for 6 hours or water as negative control.

Microglia or astrocytes were stimulated with $100 \mathrm{nM} \mathrm{FICZ} \mathrm{and} \mathrm{1 \%} \mathrm{DMSO} \mathrm{as} \mathrm{control} \mathrm{for} 6 \mathrm{~h}$. After FICZ stimulation, astrocytes were lysed in RLT lysis buffer. Subsequently, RNA was isolated and transcribed into cDNA followed by quantitative PCR. 


\subsubsection{Quantitative real-time PCR analysis}

RNA was isolated from purified astrocytes or microglia using the RNeasy Mini Kit (Qiagen) according to the manufacturer's instructions. Thereafter, RNA was transcribed into complementary DNA (cDNA) using the High-Capacity cDNA Reverse Transcription Kit with RNase Inhibitor (Applied biosystems) according to the manufacturer's protocol.

qPCR was performed with the 2x qPCRBIO Probe Mix Lo-ROX (Nippon Genetics) and TaqMan Gene Expression Assays (Life Technologies). After pre-dilution, $5 \mu \mathrm{l}$ (6.5 - $19.5 \mathrm{ng}$ ) cDNA was used for each qPCR reaction. GAPDH was used as endogenous control.

Each sample was measured in triplicates. To exclude disparities due to pipetting errors, samples with a standard deviation (SD) higher than 0.4 were excluded from further calculation. In experiments with astrocytes from Aldh1l1-AhR mice, B2m was examined additionally as a second housekeeping gene. $C_{T}$ values for expression of CYP1A1 were normalized to $C_{T}$ values of GAPDH to calculate $\Delta C_{T}$ values ( $C_{T}$ CYP1A1 $\left.-C_{T} G A P D H\right) . \Delta \Delta C_{T}$ values were calculated by subtraction of the $\Delta \mathrm{C}_{\mathrm{T}}$ value of control-treated astrocytes from $\Delta \mathrm{C}_{\mathrm{T}}$ values obtained from LAQ- or FICZ-treated cells. The expression level of each transcript is indicated as the fold change compared to the calibrator.

\subsubsection{Statistical analysis}

All statistics were calculated using the GraphPad Prism 6 software. The data were first tested for normal distribution with the D'Agostino \& Pearson omnibus normality test. For two experimental groups, unpaired t test was used for parametric values, while the Mann-Whitney test was performed for non-parametric values. For more than two groups with one factor, the Kruskal-Wallis test with Dunn's post test was carried out for non-parametric values. To compare more than two groups with two factors, the two-way ANOVA with Sidak's multiple comparisons test was used. Statistical significance was defined by $p<0.05$. All data in text and figures are presented as mean \pm SEM. 


\section{RESULTS}

\subsection{Part 1: The role of astrocytic and microglial AhR in CNS demyelination}

\subsubsection{AhR deletion in astrocytes}

\subsubsection{GFAP-AhR mice}

\subsection{Expression of the AhR transcriptional target CYP1A1 is decreased in GFAP-AhR deficient astrocytes after FICZ stimulation}

To generate astrocyte-specific AhR knockout mice, GFAP-Cre mice, expressing the Cre recombinase under control of a mouse glial fibrillary acidic protein (mGFAP) promoter sequence, were crossed with AhR LoxP (floxed) mice. To verify efficient AhR deletion in GFAPpositive astrocytes in vitro, glial cells were isolated from the brains of newborn GFAP-Cre/+ $A h R^{f l / f l}$, in the following referred to as GFAP-AhR, and littermate control mice. Cells were purified by deletion of non-target cells such as microglia (negative selection) using magneticactivated cell sorting (MACS). Before cell separation, primary cell cultures contained about 31 - 35\% microglia (Figure 3 A). After removing all CD11b+ labeled microglia, flow cytometry analysis confirmed nearly complete depletion of microglia and about $83-89 \%$ pure astrocytes (Figure $3 \mathrm{~B}$ ).

A

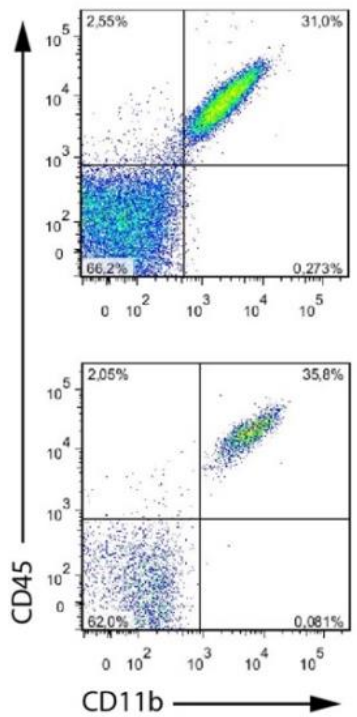

B

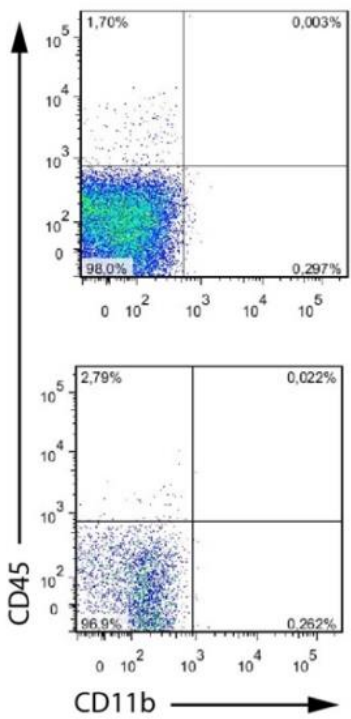

post-MACS

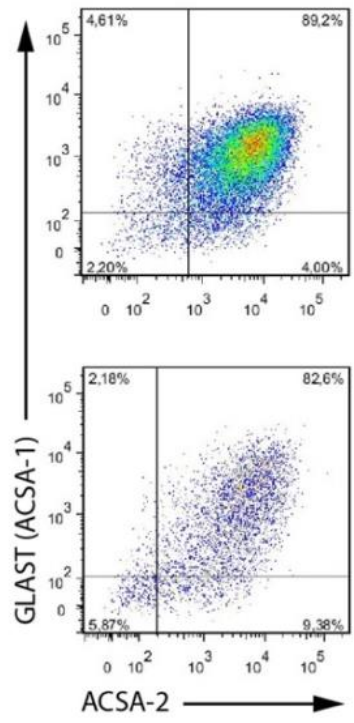

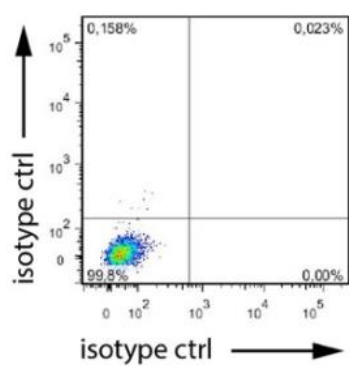

Figure 3: High astrocyte purity after MACS cell separation 
Flow cytometry analysis of microglial and astrocytic frequencies in mixed cell cultures from GFAP-AhR (upper row) and littermate control mice (lower row) before and after magnetic-activated cell sorting (MACS). Representative dot plots show the frequency of microglia $(C D 45+C D 11 b+)$ in primary cell cultures $A$ before and B after MACS cell separation. Astrocyte purity is determined by GLAST/ACSA-2 expression.

Purified astrocytes were stimulated with the AhR-specific and high-affinity ligand FICZ at a concentration of $100 \mathrm{nM}$ or vehicle (1\% DMSO) for $6 \mathrm{~h}$ and RNA was isolated. The expression levels of the AhR target gene CYP1A1 showed a 63-fold increase in control astrocytes after FICZ compared to control treatment. By contrast, GFAP-AhR astrocytes displayed an upregulation of only about 12-fold in CYP1A1 expression upon FICZ treatment, far lower than the response of control astrocytes. Hence, the response of GFAP-AhR astrocytes upon FICZ treatment was diminished by about 51-fold compared to control astrocytes (treatment: $F(1,26)=21.16 ; p<0.0001$; genotype: $F(1,26)=10.05 ; p=0.0039$, two-way ANOVA with Sidak's multiple comparisons test) (Figure 4).

\section{CYP1A1}

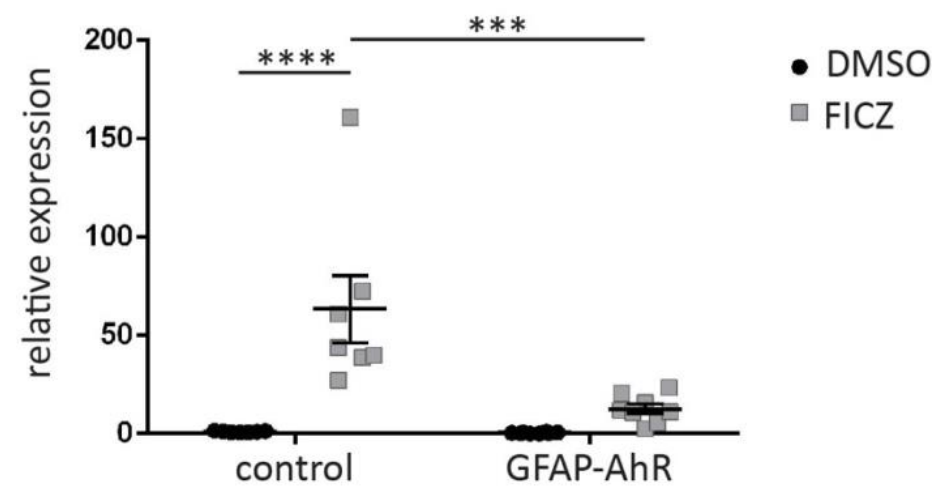

Figure 4: Loss of AhR in GFAP-positive astrocytes leads to decreased CYP1A1 response upon FICZ stimulation

Transcript levels were measured using quantitative real-time PCR for CYP1A1 expression relative to GAPDH in RNA isolated from purified astrocytes from GFAP-Cre/+ AhR ${ }^{\text {fl/fl }}$ mice $(n=8)$ and littermate controls $(n=7)$. Cells were treated with $100 \mathrm{nM} \mathrm{FICZ}$ or vehicle (1\% DMSO) for $6 \mathrm{~h}$. Data are representative of 2 independent experiments. Each sample was measured in triplicates. Data are presented as mean \pm SEM. Statistical significance was determined by two-way ANOVA followed by Sidak's post hoc test. ${ }^{* * *} p<0.001,{ }^{* * * *} p<0.0001$.

\subsection{Clinical observations in GFAP-AhR knockout mice during cuprizone-induced demyelination}

GFAP-AhR mice were normal in size and appearance at birth and adult age. To test the role of astrocytic AhR in CNS demyelination, 8- to 24-week-old knockout and control mice 
(Cre-negative littermates) were fed with $0.25 \%$ cuprizone for 6 weeks and evaluated clinically after each week of cuprizone treatment. Especially at the beginning of the cuprizone diet, both groups demonstrated some lethargy. Overall, GFAP-AhR mice displayed no differences in behavior compared to controls. Both GFAP-AhR and control mice showed weight loss after one week of cuprizone challenge, followed by steady weight gain after two weeks of cuprizone intake until the end of the experiment. AhR-deficient mice displayed constantly lower body weights compared to control animals, albeit without significant difference (Figure 5).

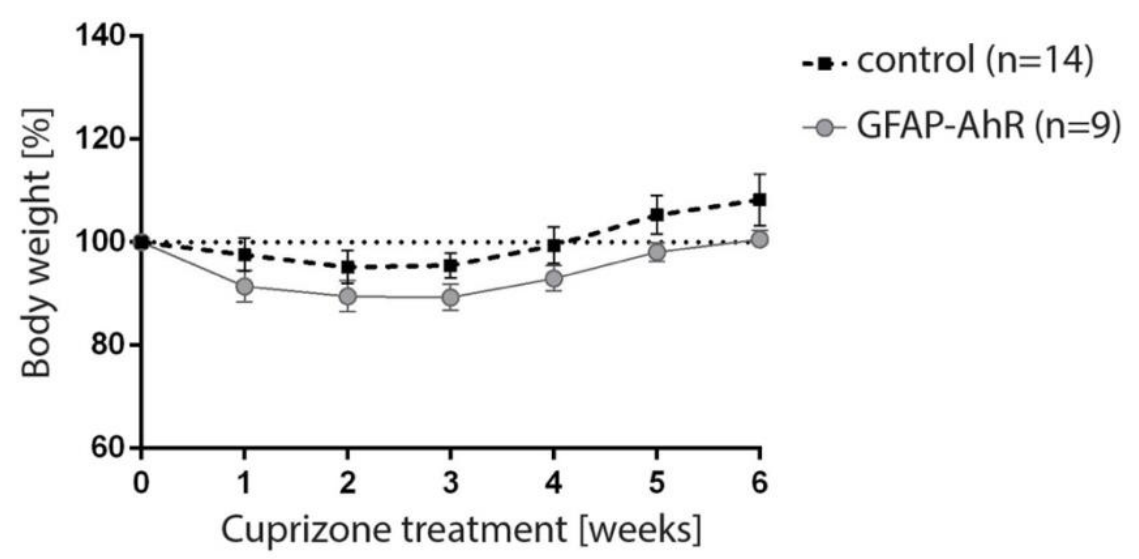

Figure 5: No significant difference in body weights of GFAP-AhR mice fed with $0.25 \%$ cuprizone for 6 weeks compared to control mice

Body weight curves of GFAP-AhR $(n=9)$ and littermate control mice $(n=14)$ relative to their initial weights at experiment start. Animals of both genotypes were at least 8 weeks old. Data originate from 2 independent experiments and are presented as mean \pm SEM. Statistical significance was tested by two-way ANOVA with Sidak's post hoc test.

\subsection{GFAP-AhR knockout mice do not show any alteration in the extent of demyelination and the density of mature oligodendrocytes}

Feeding the copper chelator cuprizone leads to severe oligodendrocyte damage with concomitant microglial activation and severe astrocytosis. To determine whether AhR in GFAP-positive astrocytes plays a role during cuprizone intoxication, brain sections of mice with astrocytic AhR deletion and control mice were examined and analyzed for demyelination of the corpus callosum by LFB-PAS staining in which the myelin appears blue. Demyelination was evaluated using an extended semi quantitative scoring system: no (0), minimal (0.5), $<33 \%$ (1), 33-66\% (2), and > 66\% demyelination (3). Both GFAP-AhR and control mice displayed extensive callosal demyelination (Figure $6 \mathrm{~A}, \mathrm{~B}$ ). The demyelination scores were 
similar in animals with loss of AhR in GFAP-positive astrocytes (score $2.2 \pm 0.2$ ) compared to controls (score $2.0 \pm 0.2$ ) (Figure $6 \mathrm{C}$ ).
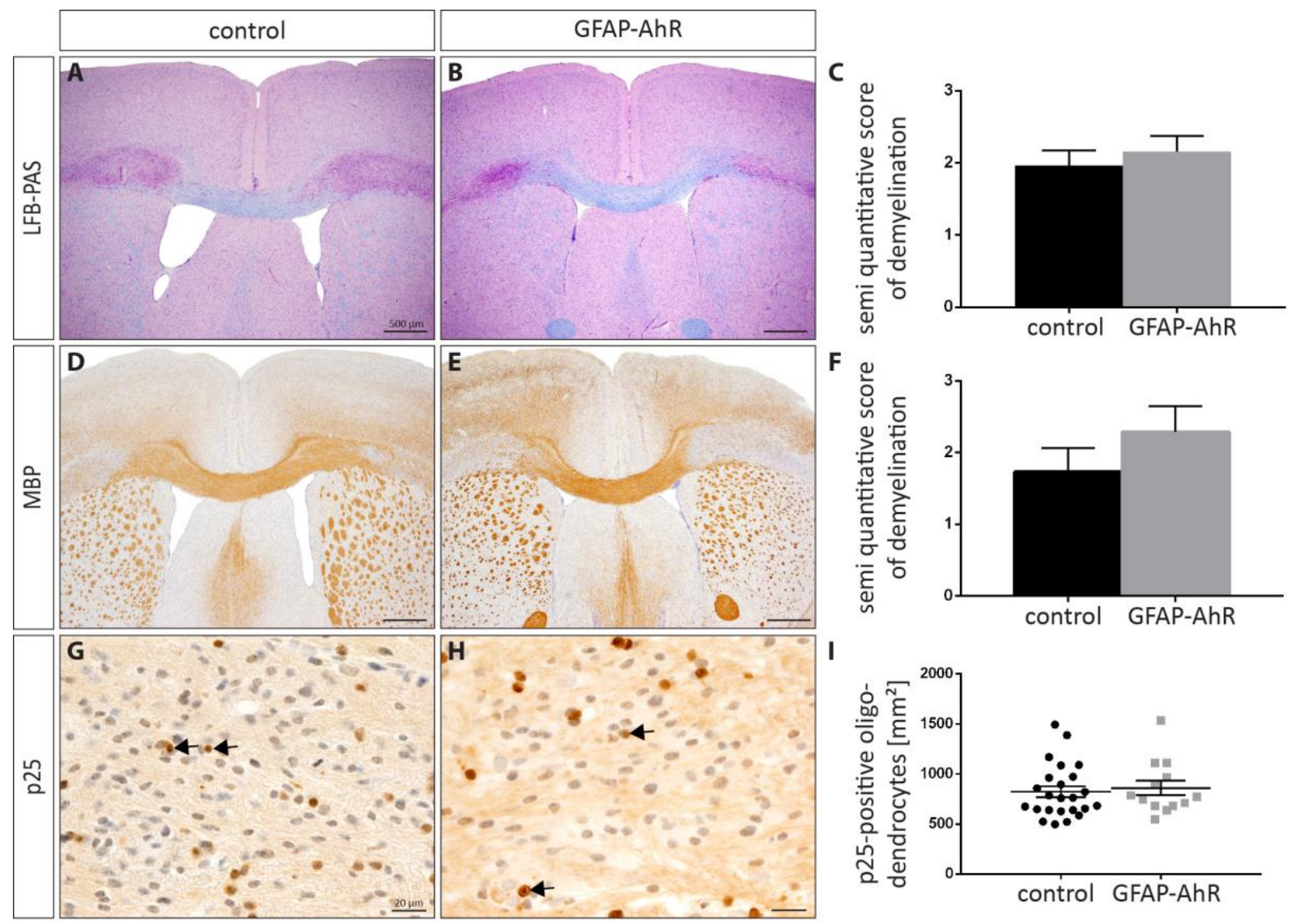

Figure 6: Cuprizone feeding for 6 weeks induces strong demyelination with comparable numbers of mature oligodendrocytes in both GFAP-AhR and control mice

Histology and immunohistochemistry using sections from GFAP-AhR and control mice (Cre-negative littermates) after 6 weeks of cuprizone intoxication with respective quantifications in the entire corpus callosum. (A, B) LFBPAS (D, E) MBP and (G, H) TPPP/p25 IHC of representative sections from control and GFAP-AhR mice, respectively. (C) Quantification of demyelination in control $(n=25)$ and GFAP-AhR mice ( $n=13)$ by scoring LFBPAS staining. (F) Quantification of demyelination in control $(n=15)$ and GFAP-AhR mice $(n=7)$ by scoring MBP sections. (I) Quantification of TPPP/p25 mature oligodendrocytes of control $(n=24)$ and GFAP-AhR $(n=13)$ mice. A score of 0 represents no demyelination and a score of 3 illustrates complete demyelination. The LFB-PAS score was analyzed by unpaired $t$ test (C). MBP and TPPP/p25 staining data were analyzed by Mann-Whitney test (F, I). Data are presented as mean \pm SEM. Arrows indicate TPPP $/ \mathrm{p} 25^{+}$mature oligodendrocytes (G, H). Scale bars (A, B, D, E) $500 \mu \mathrm{m}$ (G, H) $20 \mu \mathrm{m}$.

Brain sections were further stained and scored for myelin basic protein (MBP). The amount of myelin present in the mouse corpus callosum, evaluated by MBP, revealed similar results to LFB-PAS staining data. Semi quantitative analyses confirmed no alteration in the levels of MBP between GFAP-AhR (score $2.3 \pm 0.4$ ) and control animals (score $1.7 \pm 0.3$ ) (Figure 6 D-F). 
By assessing TPPP/p25, a marker for mature oligodendrocytes, similar numbers of mature oligodendrocytes were observed in the corpora callosa of both groups (GFAP-AhR mice: 859.5 \pm 74.1 vs. controls: $821.7 \pm 54.4 \mathrm{TPPP} / \mathrm{p}^{2} 5^{+}$cells per $\mathrm{mm}^{2}$ ) (Figure $6 \mathrm{G}-\mathrm{I}$ ).

\subsection{No change in microglial activation and acute axonal damage in GFAP-AhR mice after cuprizone feeding}

To evaluate microglial activity of GFAP-AhR mice fed with $0.25 \%$ cuprizone for 6 weeks, the frequency of callosal Mac3-positive microglia was evaluated. Quantification of Mac3positive cells revealed no difference between GFAP-AhR mice $\left(1321 \pm 225.2 \mathrm{Mac3}^{+}\right.$cells per $\mathrm{mm}^{2}$ ) and their littermates (1185 $\pm 160.0 \mathrm{Mac3}^{+}$cells per $\mathrm{mm}^{2}$ ) (Figure $7 \mathrm{~A}-\mathrm{C}$ ).

To determine the effect of astrocytic AhR deletion on axonal integrity, we examined acutely damaged axons characterized by swelling and accumulation of amyloid precursor protein (APP). There was no modification in acute axonal injury of GFAP-AhR mice (531.6 \pm 79.1 $\mathrm{APP}^{+}$spheroids per $\mathrm{mm}^{2}$ ) compared to controls (490.6 $\pm 82.7 \mathrm{APP}^{+}$spheroids per $\mathrm{mm}^{2}$ ) (Figure 7 F).
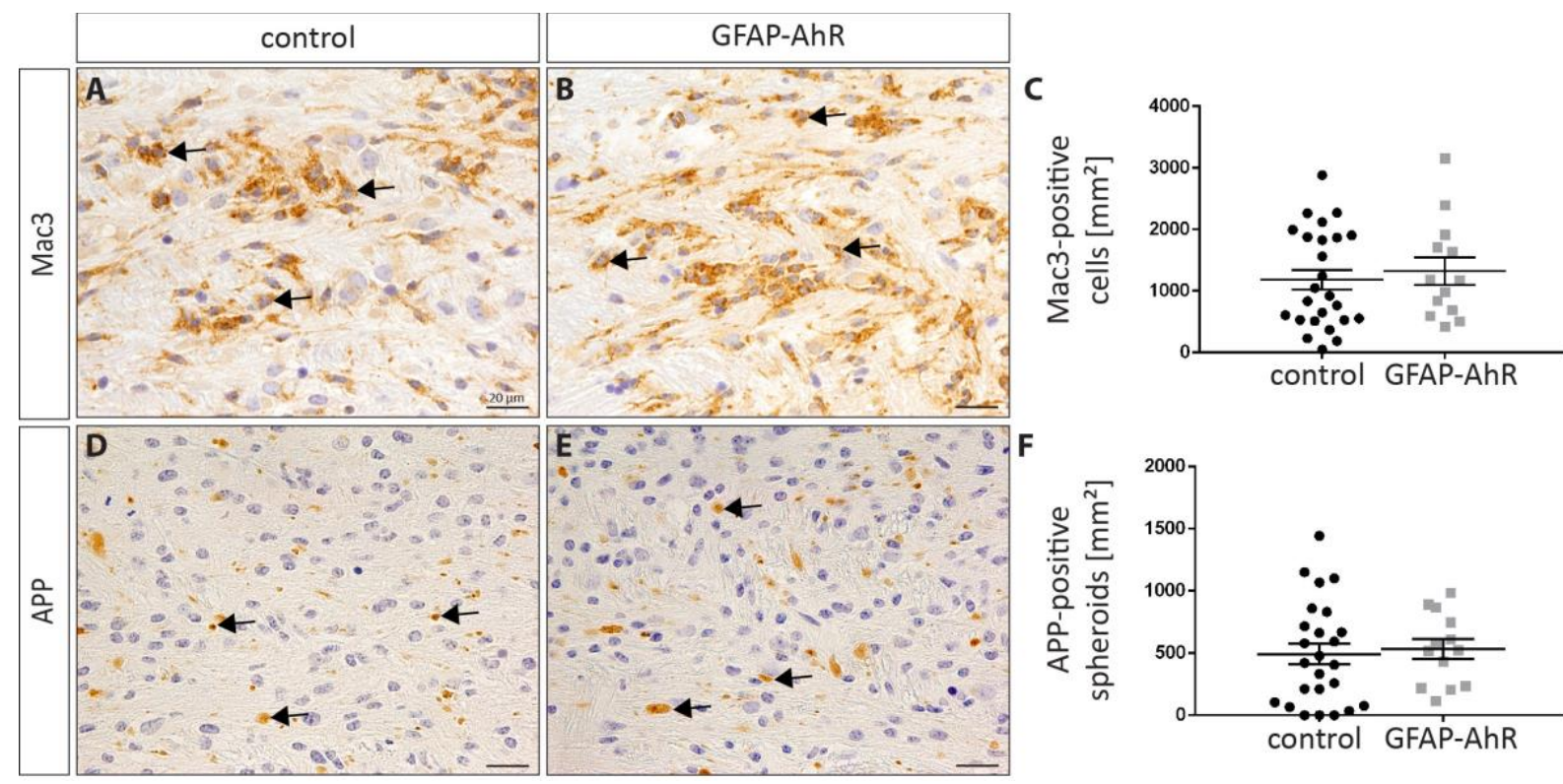

Figure 7: Extensive accumulation of microglia and axonal spheroids in the corpora callosa of both GFAP-AhR and control mice during demyelination

(A, B) Mac3 and (D, E) APP IHC of representative sections from GFAP-Cre/+ AhR $\mathrm{R}^{\mathrm{fl} / \mathrm{fl}}(n=13)$ and littermate control mice $(n=25)$, respectively, after 6 weeks of cuprizone intoxication. (C) Quantification of $\mathrm{Mac}^{+}$activated microglia in the corpus callosum of respective animals. (F) Quantification of acute axonal damage by counting $\mathrm{APP}^{+}$axonal spheroids. Data are presented as mean \pm SEM and were analyzed by unpaired t test (C, F). Arrows indicate $\mathrm{Mac}^{+}$activated microglia (A, B) and APP+ axonal spheroids (D, E). Scale bars (A, B, D, E) $20 \mu \mathrm{m}$. 


\subsubsection{Aldh1l1-AhR mice}

\subsection{CYP1A1 mRNA levels are reduced in stimulated AhR-deleted Aldh1l1+ astrocytes}

Aldh1l1 has been found to be specifically expressed in astrocytes with a considerably broader expression pattern than GFAP (Cahoy et al. 2008). Since GFAP only labels a limited number of astrocytes, we additionally used aldehyde dehydrogenase 1 family member L1 (Aldh1l1) as a second astroglial marker targeting also cortical astrocytes. We generated a targeted AhR deletion in Aldh1l1-positive astrocytes by crossbreeding Aldh1l1-CreERT2 mice with AhR LoxP (floxed) mice.

In order to assess whether AhR-deficient astrocytes still responded to the AhR ligand FICZ, we performed in vitro experiments with murine primary astrocytes from Aldh1l1CreERT2/+ AhR ${ }^{\text {fl/fl }}$ mice, hereinafter referred to as Aldh1l1-AhR. The prodrug tamoxifen, a selective estrogen response modifier (SERM), is utilized for the induction of Cre in mice and is converted into 4-hydroxytamoxifen in vivo (MacGregor and Jordan 1998). For in vitro approaches, however, the active metabolite 4-hydroxytamoxifen is used instead. Purified astrocytes from Aldh1l1-AhR and littermate control mice were therefore pre-treated with $20 \mu \mathrm{M}$ 4-hydroxytamoxifen for $24 \mathrm{~h}$ and stimulated with FICZ or vehicle (1\% DMSO) for $6 \mathrm{~h}$. RNA was isolated and qPCR studies were performed. In control astrocytes, FICZ stimulation resulted in a 56-fold upregulation of CYP1A1 mRNA expression levels compared to vehicle treatment. In AhR-deficient astrocytes, the effect was much smaller. CYP1A1 expression levels of Aldh1l1-AhR astrocytes were elevated upon FICZ treatment, with an increase of about 11-fold compared to control treatment. Thus, CYP1A1 mRNA levels measured by the response of Aldh1l1-AhR astrocytes to FICZ treatment were downregulated by about 45 -fold compared to CYP1A1 mRNA levels of FICZ-treated control astrocytes (treatment: $F(1,12)=97.35$; $p<0.0001$; genotype: $F(1,26)=46.00 ; p<0.0001$, two-way ANOVA with Sidak's multiple comparisons test) (Figure 8). 
CYP1A1

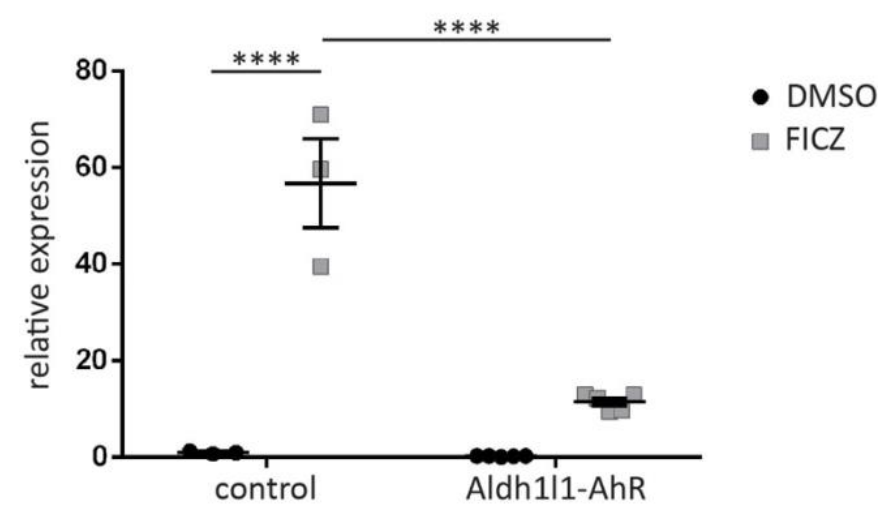

Figure 8: Reduced CYP1A1 mRNA levels in Aldh1|1 ${ }^{+}$astrocytes compared to control astrocytes after FICZ stimulation (in vitro)

Transcript levels were measured using quantitative real-time PCR for CYP1A1 expression relative to GAPDH in RNA isolated from purified astrocytes from Aldh1/1-CreERT2/+ $\operatorname{AhR}^{\mathrm{fl} / \mathrm{fl}}(n=5)$ and littermate control mice $(n=3)$. Cells were pre-treated with $20 \mu \mathrm{M}$ 4-hydroxytamoxifen for $24 \mathrm{~h}$, followed by treatment with $100 \mathrm{nM}$ FICZ or vehicle (1\% DMSO) for $6 \mathrm{~h}$. Each sample was measured in triplicates. Data are presented as mean \pm SEM. Statistical significance was determined by two-way ANOVA followed by Sidak's multiple comparisons test. $* * * * p<0.0001$.

\subsection{No clinical signs or body weight abnormalities of Aldh1/1-AhR conditional knockout mice}

To test the role of astrocytic AhR in CNS demyelination, 8- to 19-week-old Aldh1l1-AhR mice received intraperitoneal tamoxifen injections at a dosage of $75 \mathrm{mg} / \mathrm{kg}$ body weight on 5 consecutive days to achieve efficient recombination of AhR in Aldh $1 / 1^{+}$astrocytes. The following week, Aldh1I1-AhR and control mice (Cre-negative littermates) were treated with a $0.25 \%$ cuprizone diet for 6 weeks in order to induce toxic demyelination in the central nervous system. Aldh1l1-AhR mice were evaluated clinically and histologically.

After one week of cuprizone challenge, all animals showed severe weight loss (Figure 9). At a concentration of $0.25 \%$ cuprizone, 2 out of 28 mice died unexpectedly or displayed serious weight loss of more than $20 \%$ within one week and were consequently excluded from the experiment (data not shown). Thus, the mortality rate was $7 \%$. While control mice (Crenegative littermates) demonstrated a continuous weight gain, Aldh1l1-AhR mice presented a slightly delayed weight gain starting from cuprizone treatment week 2 . Apart from weight loss and minimally reduced activity, no further clinical signs were observed. 


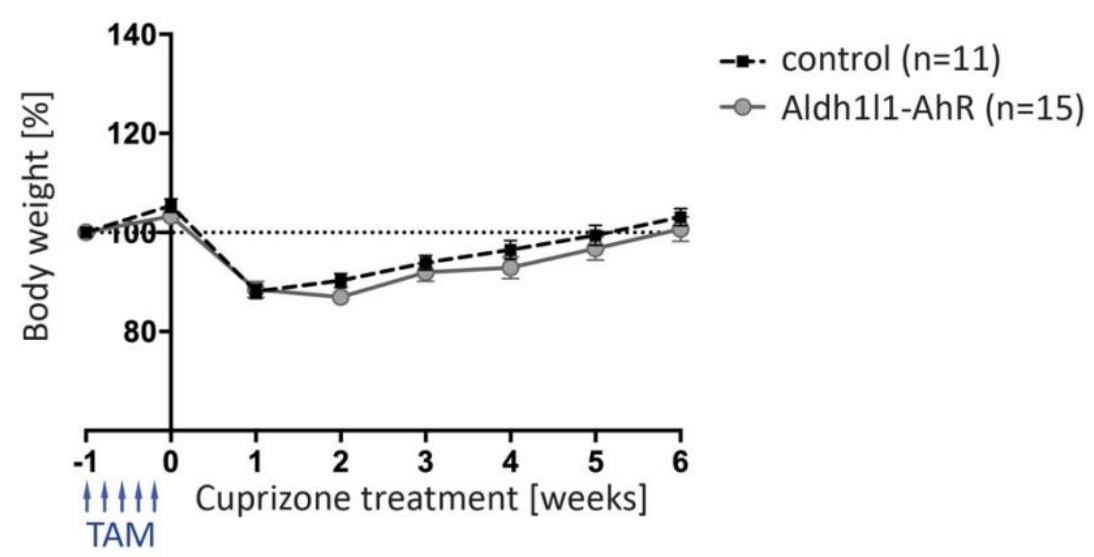

Figure 9: No alteration in body weights of Aldh1l1-AhR compared to control mice after cuprizone intoxication

Body weight curves of Aldh111-AhR $(n=15)$ and littermate control mice $(n=11)$ relative to their initial weights at experiment start 1 week before cuprizone challenge. Pre-treatment included intraperitoneal tamoxifen injections at a concentration of $75 \mathrm{mg} / \mathrm{kg}$ body weight on 5 consecutive days before mice were fed with a $0.25 \%$ cuprizone diet for 6 weeks. Data are presented as mean \pm SEM and originate from 2 independent experiments. Statistical significance was tested by two-way ANOVA with Sidak's post hoc test.

\subsection{Cuprizone intoxication for 6 weeks does not modify the extent of demyelination and numbers of mature oligodendrocytes in Aldh1l1- AhR mice}

Histological evaluation of LFB-PAS confirmed pronounced demyelination in the corpora callosa of both animal groups (Figure $10 \mathrm{~A}, \mathrm{~B}$ ). The demyelination scores of Aldh1l1-AhR mice (score $2.3 \pm 0.2$ ) were not significantly different from control mice $(1.8 \pm 0.2)$, but displayed a trend towards a greater extent of demyelination ( $p=0.0669$, unpaired $t$ test) (Figure $10 \mathrm{C}$ ).

Immunohistochemistry (IHC) for TPPP/p25 revealed similar densities of mature oligodendrocytes in the corpora callosa of astrocyte-specific AhR knockout mice and littermate controls (Aldh1l1-AhR: $971.8 \pm 44.4$ vs. control: $1082 \pm 93.6$ TPPP/p25 cells per $\mathrm{mm}^{2}$ ) (Figure $10 \mathrm{D}-\mathrm{F}$ ). 

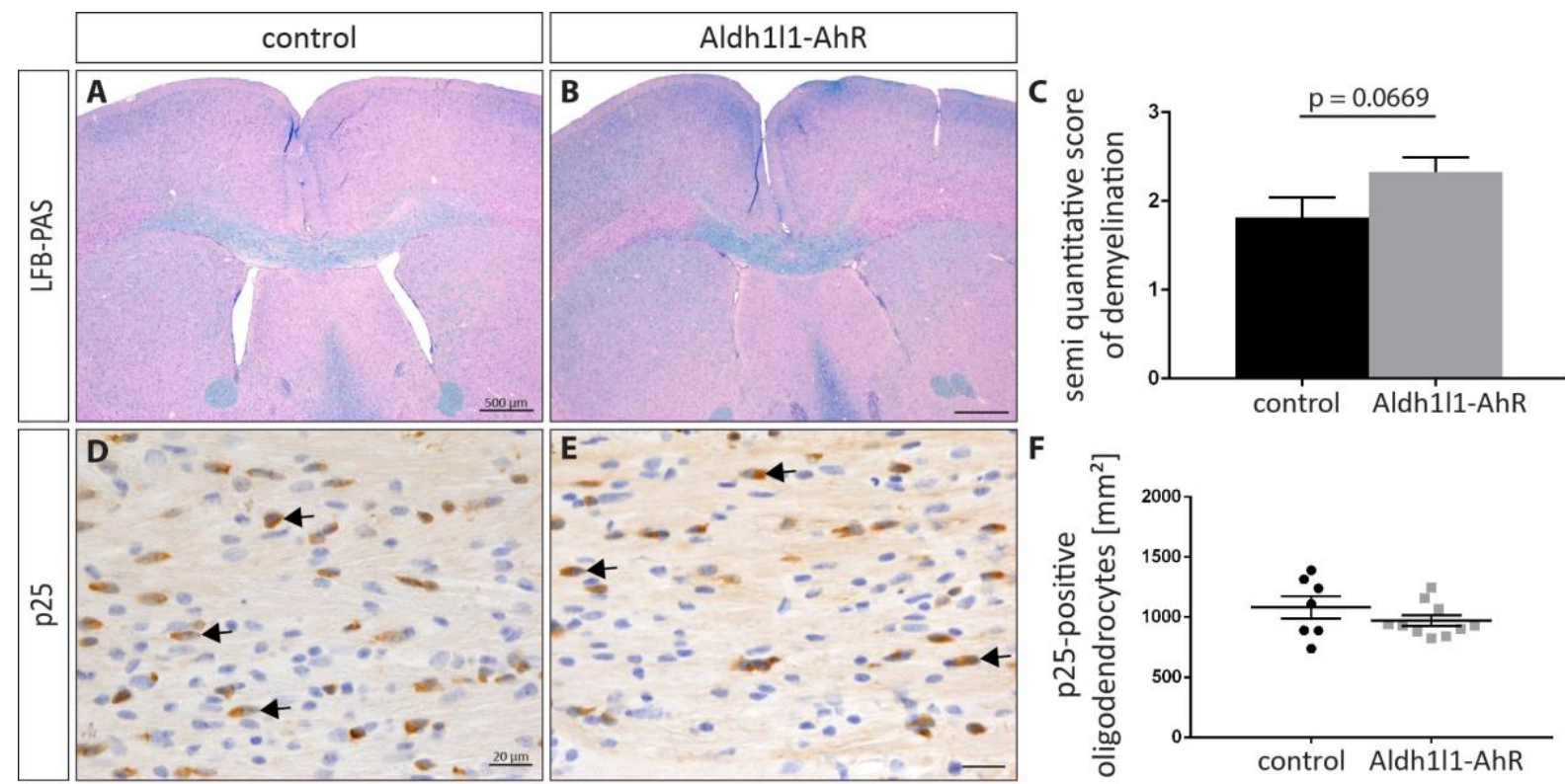

Figure 10: No change in demyelination or densities of mature oligodendrocytes between Aldh1/1-AhR and control mice

Histology and immunohistochemistry using sections of corpora callosa from Aldhl1-AhR and littermate control mice after 6 weeks of cuprizone intoxication with respective quantifications. (A, B) LFB-PAS and (D, E) TPPP/p25 IHC of representative sections from control and Aldh1l1-AhR mice, respectively. (C) Quantification of demyelination by scoring LFB-PAS staining in the entire corpus callosum of control $(n=11)$ and Aldh1l1-AhR mice $(n=15)$. (F) Quantification of TPPP/p25+ mature oligodendrocytes in the corpus callosum of control $(n=7)$ and Aldh1l1-AhR mice $(n=10)$. A score of 0 represents no demyelination and a score of 3 illustrates complete demyelination. The LFB-PAS scores were analyzed by unpaired t test (C). Quantifications of TPPP/p25 were analyzed by Mann-Whitney test (F). Data are presented as mean \pm SEM. Arrows indicate TPPP/p $25^{+} \mathrm{mature}$ oligodendrocytes (D, E). Scale bars (A, B) $500 \mu \mathrm{m}$ (D, E) $20 \mu \mathrm{m}$.

\subsection{Microglial density and acute axonal damage are unaltered in Aldh1l1-AhR mice compared to control mice}

Microglia accumulation after 6 weeks of toxic demyelination was analyzed by immunohistochemistry for Mac3 on brains sections from Aldh1l1-AhR mice (Figure 11 B) and littermate control mice (Figure $11 \mathrm{~A}$ ). Quantitative evaluation indicated no difference between animals with AhR-competent and -deficient Aldh1l1 ${ }^{+}$astrocytes (Aldh1l1-Ahr: $1007 \pm 85.4$ vs. control: $806.9 \pm 103.5 \mathrm{Mac3}^{+}$cells per $\mathrm{mm}^{2}$ ) (Figure $11 \mathrm{C}$ ). However, a trend towards an increase in the numbers of $\mathrm{Mac}^{+}$cells was observed in Aldh1l1-AhR mice $(p=0.0869$, MannWhitney test). To determine acute axonal damage, sections were stained for APP to detect swollen and accumulated amyloid precursor protein, indicated as brown accumulated structures (Figure $11 \mathrm{D}, \mathrm{E}$ ). Aldh1|1-AhR mice displayed no difference in the number of APPpositive axonal spheroids $\left(447.7 \pm 111.1 \mathrm{APP}^{+}\right.$spheroids per $\mathrm{mm}^{2}$ ) compared to control mice $\left(286.4 \pm 118.7 \mathrm{APP}^{+}\right.$spheroids per $\left.\mathrm{mm}^{2}\right)$ (Figure $\left.11 \mathrm{~F}\right)$. 

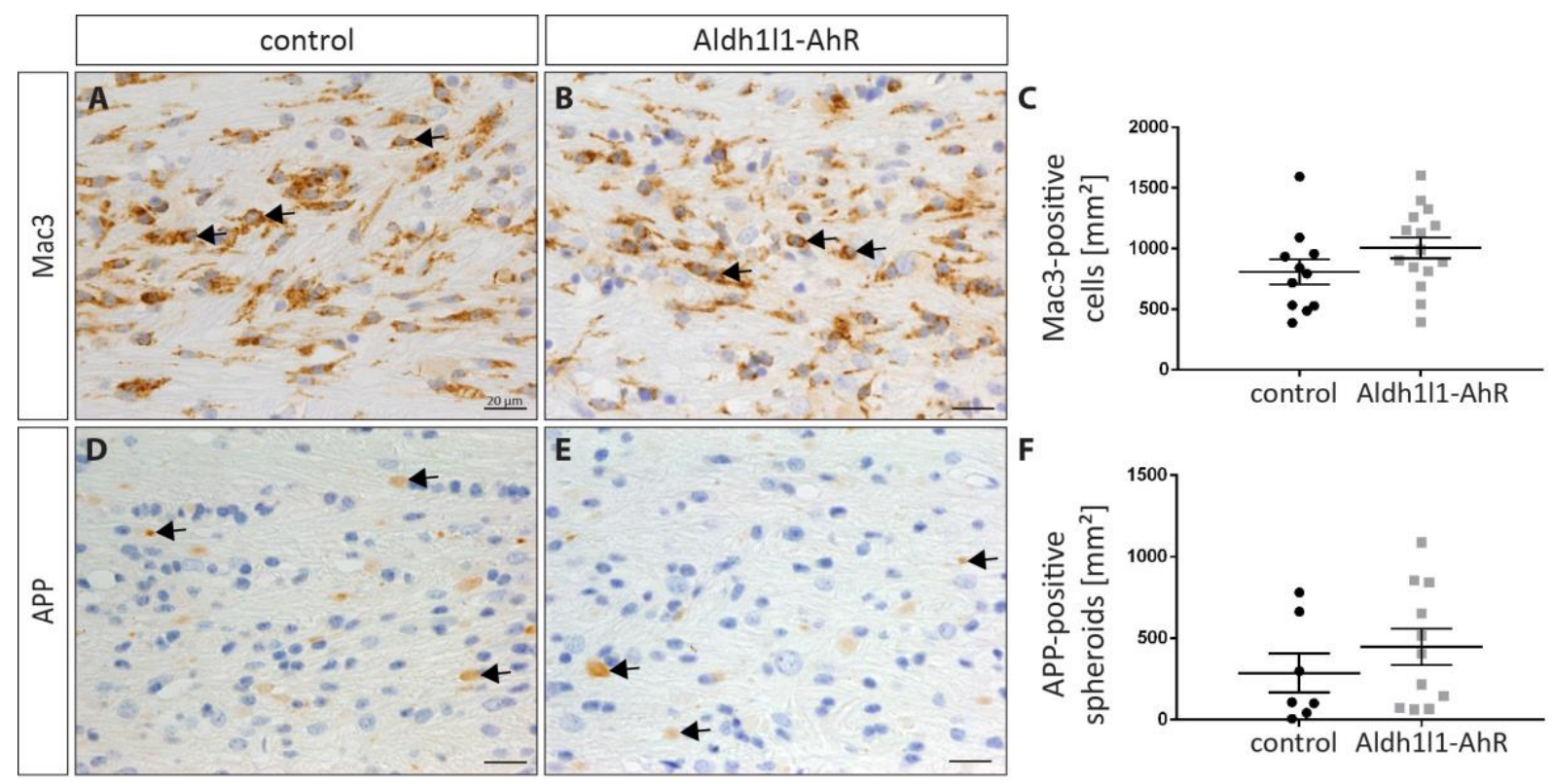

Figure 11: Numbers of $\mathrm{Mac3}^{+}$activated microglia and $\mathrm{APP}^{+}$axonal spheroids are comparable in the corpus callosum of Aldh1l1-AhR mutants and littermate controls after 6 weeks of cuprizone exposure

(A, B) Mac3 and (D, E) APP IHC of representative sections from Aldh1l1-AhR and littermate control mice, respectively after 6 weeks of cuprizone treatment. (C) Quantification of $\mathrm{Mac}^{+}$activated microglia in the corpus callosum of Aldh1l1-AhR $(n=15)$ and control mice $(n=11)$. (F) Quantification of APP ${ }^{+}$spheroids in control $(n=7)$ and Aldh1l1-AhR mice $(n=11)$. Data are presented as mean \pm SEM and were analyzed by Mann-Whitney test (C, F). Arrows indicate Mac3 ${ }^{+}$activated microglia (A, B) and APP+ axonal spheroids (D, E). Scale bars (A, B, D, E) $20 \mu \mathrm{m}$.

\subsubsection{AhR deletion in microglia}

\subsubsection{Clinical evaluation of CX3CR1-AhR conditional knockout mice}

To investigate the impact of AhR deletion in microglia during CNS demyelination, we utilized the cuprizone model in mice with microglia-specific AhR deletion. To generate CX3CR1 cell-specific AhR conditional knockout mice, CX3CR1-CreERT2 mice expressing the Cre recombinase under control of the CX3CR1 promoter were crossed with AhR LoxP (floxed) mice. Adult CX3CR1-CreERT2/+ AhR fl/fl mice, abbreviated as CX3CR1-AhR in the following, were treated with tamoxifen at the age of 9 to 16 weeks. Tamoxifen was administered at a concentration of $75 \mathrm{mg} / \mathrm{kg}$ via intraperitoneal injections on 5 consecutive days to accomplish Cre-mediated recombination. Toxic demyelination was induced by feeding $0.25 \%$ of the copper chelator cuprizone for 6 weeks.

Clinical observations revealed no behavioral deficits in CX3CR1-AhR mice compared to littermate control animals. In both groups, mice gained weight during tamoxifen pretreatment, lost weight until week 2 of the cuprizone diet and steadily gained weight until the 
end of the experiment (Figure 12). AhR-deficient mice showed no difference in body weights to control mice and reached their initial body weights at week 6 , whereas control mice reached their initial body weights one week earlier.

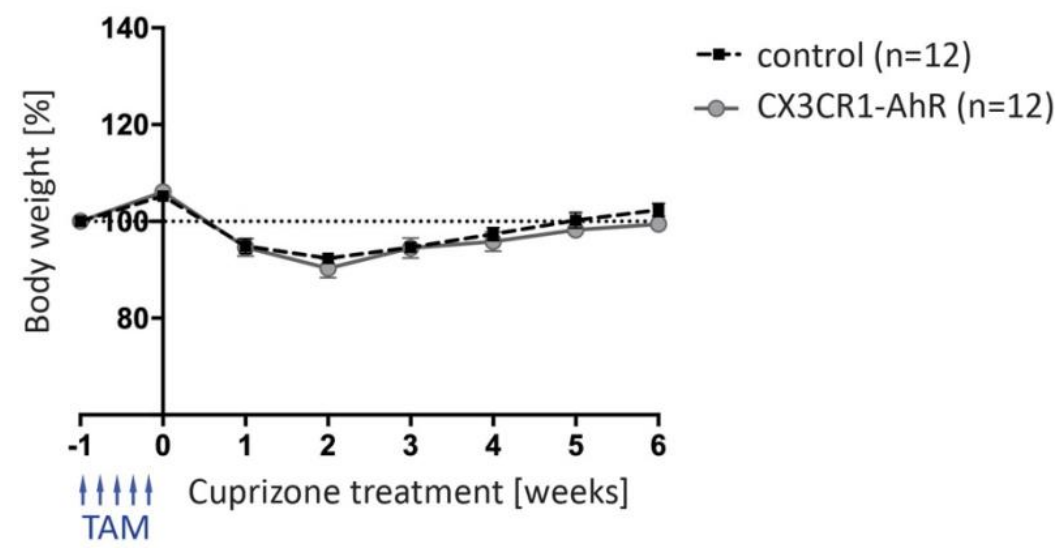

Figure 12: Body weights of CX3CR1-AhR mice are unaffected by cuprizone intoxication compared to control mice

Body weight curves of CX3CR1-AhR ( $n=12)$ and littermate control mice $(n=12)$ relative to their initial weights at experiment start one week prior to cuprizone treatment. All mice received intraperitoneal tamoxifen injections ( $75 \mathrm{mg} / \mathrm{kg}$ body weight) on 5 consecutive days before a $0.25 \%$ cuprizone diet was administered for 6 weeks. Animals of both genotypes were at least 9 weeks old at experiment start. Data originate from 2 independent experiments. Data are shown as mean \pm SEM and were analyzed using two-way ANOVA with Sidak's post test.

\subsubsection{Microglia-specific AhR deletion has no influence on demyelination but on the density of mature oligodendrocytes after 6 weeks of cuprizone treatment}

To test whether microglial AhR plays a role during CNS demyelination, histological evaluation was performed on sections stained with Luxol Fast Blue-Periodic Acid Schiff (LFBPAS) to determine demyelination using an extended semi quantitative scoring system. Control mice (score $2.1 \pm 0.3$ ) and mice with microglial AhR deletion (score $1.7 \pm 0.2$ ) displayed no significant difference in demyelination (Figure $13 \mathrm{~A}-\mathrm{C}$ ). 

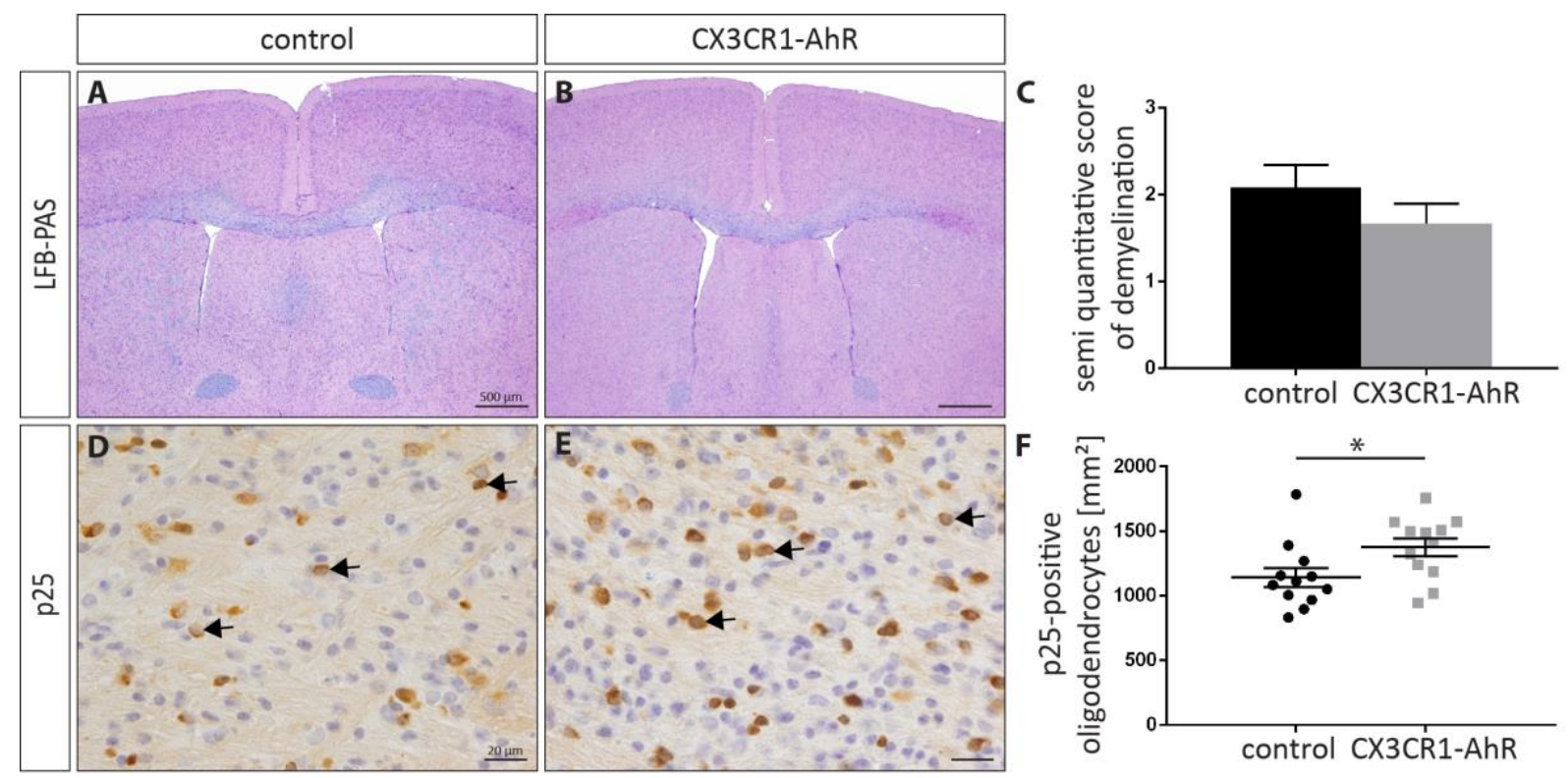

Figure 13: Cuprizone treatment does not modify the extent of demyelination but leads to higher numbers of mature oligodendrocytes in CX3CR1-AhR mice versus littermate controls Histological and immunohistochemical evaluation of sections from CX3CR1-AhR $(n=12)$ and littermate control mice $(n=12)$ after 6 weeks of cuprizone feeding. (A, B) LFB-PAS and (D, E) TPPP/p25 IHC of representative sections of corpora callosa from control and CX3CR1-AhR mice, respectively. (C) Quantification of the extent of demyelination by scoring LFB-PAS staining in the entire corpus callosum. A score of 0 indicates complete myelination, a score of 3 demonstrates complete demyelination. (F) Quantification of TPPP/p25 mature oligodendrocytes in the corpus callosum of respective animals. LFB-PAS staining data were analyzed by unpaired $t$ test (C). TPPP/p25 staining data were evaluated by Mann-Whitney test $\left({ }^{*} p<0.05\right)$ (F). Data are presented as

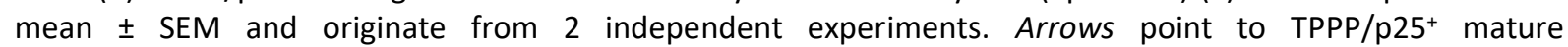
oligodendrocytes (D, E). Scale bars (A, B) $500 \mu \mathrm{m}$ (D, E) $20 \mu \mathrm{m}$.

To evaluate whether microglial AhR deletion has an effect on the density of mature oligodendrocytes during cuprizone-induced demyelination, TPPP/p25-positive cells were counted and compared between both groups. The frequency of mature oligodendrocytes was significantly higher in animals with AhR-deleted CX3CR1 $1^{+}$microglia $\left(1377 \pm 69.4 \mathrm{TPPP} / \mathrm{p} 25^{+}\right.$ cells per $\mathrm{mm}^{2}$ ) compared to mice with AhR-competent microglia (1143 $\pm 73.3 \mathrm{TPPP} / \mathrm{p} 25^{+}$cells per $\mathrm{mm}^{2} ; \mathrm{p}=0.0241$, Mann-Whitney test) (Figure $13 \mathrm{D}-\mathrm{F}$ ).

\subsubsection{Microglia-specific AhR deletion does not affect microglial activation or acute axonal damage during cuprizone intake}

Although mice with AhR-deficient microglia displayed higher numbers of mature oligodendrocytes compared to littermate control mice, similar dimensions of callosal demyelination could be observed. To further investigate whether these findings are accompanied by alterations in microglial infiltration or acute axonal damage, sections of corpora callosa were stained for Mac3 and APP to evaluate activated microglia and acute 
axonal injury, respectively. However, neither microglial activation (CX3CR1-AhR: $831.0 \pm 105.2$

vs. control mice: $1008 \pm 160.4 \mathrm{Mac3}^{+}$cells per $\mathrm{mm}^{2}$ ) (Figure $14 \mathrm{~A}-\mathrm{C}$ ), nor acute axonal damage (CX3CR1-AhR: $157.6 \pm 39.9$ vs. control mice: $218.5 \pm 42.4 \mathrm{APP}^{+}$spheroids per $\mathrm{mm}^{2}$ ) (Figure 14 D-F) differed between CX3CR1-AhR and littermate control mice.
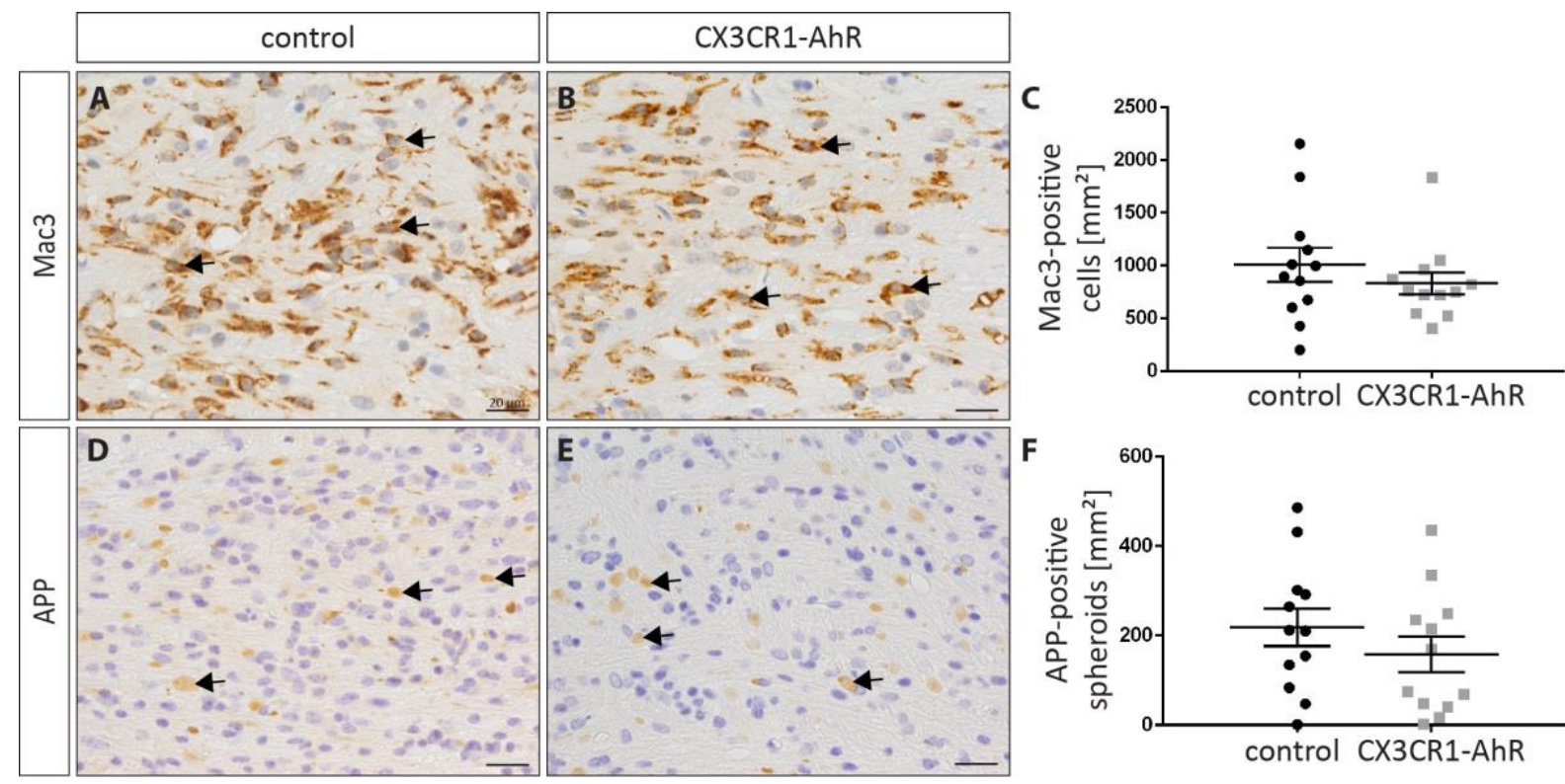

Figure 14: Microglia-specific AhR deletion has no effect on acute axonal damage or microglial infiltration into the corpus callosum

(A, B) Mac3 and (D, E) APP IHC of brain sections from CX3CR1-AhR ( $n=12)$ and littermate control mice $(n=12)$ 6 weeks after cuprizone treatment. Representative sections are shown. (C) Quantification of Mac3-positive cells for CX3CR1-AhR and control mice. (F) Quantification of APP-positive axonal spheroids in the corpora callosa of respective animals. Data derive from 2 independent experiments and are presented as mean \pm SEM. Mac3 data were analyzed by Mann-Whitney test (C), APP staining data were evaluated by unpaired $t$ test. Arrows indicate Mac3-positive activated microglia (A, B) and APP-positive axonal spheroids (D, E). Scale bars (A, B, D, E) $20 \mu \mathrm{m}$.

\subsubsection{Cuprizone challenge for 3 weeks reveals a higher density of mature oligodendrocytes and less demyelination in CX3CR1-AhR mice}

The data presented above demonstrate that mice with microglia-specific AhR deletion showed significantly higher numbers of mature oligodendrocytes versus control mice after 6 weeks of cuprizone. Hence, we evaluated whether this effect is already visible in these mice after a shorter period of toxin-induced demyelination.

Among all CX3CR1-AhR mice and littermate controls, none of the animals showed behavioral abnormalities. LFB-PAS-labeled sections were prepared to assess the extent of demyelination after 3 weeks of cuprizone intake. CX3CR1-AhR mice demonstrated 
significantly less demyelination (score $0.5 \pm 0.0$ ) relative to control mice (score $1.1 \pm 0.2$; $\mathrm{p}=0.033$, Mann-Whitney test) (Figure $15 \mathrm{~A}-\mathrm{C}$ ).
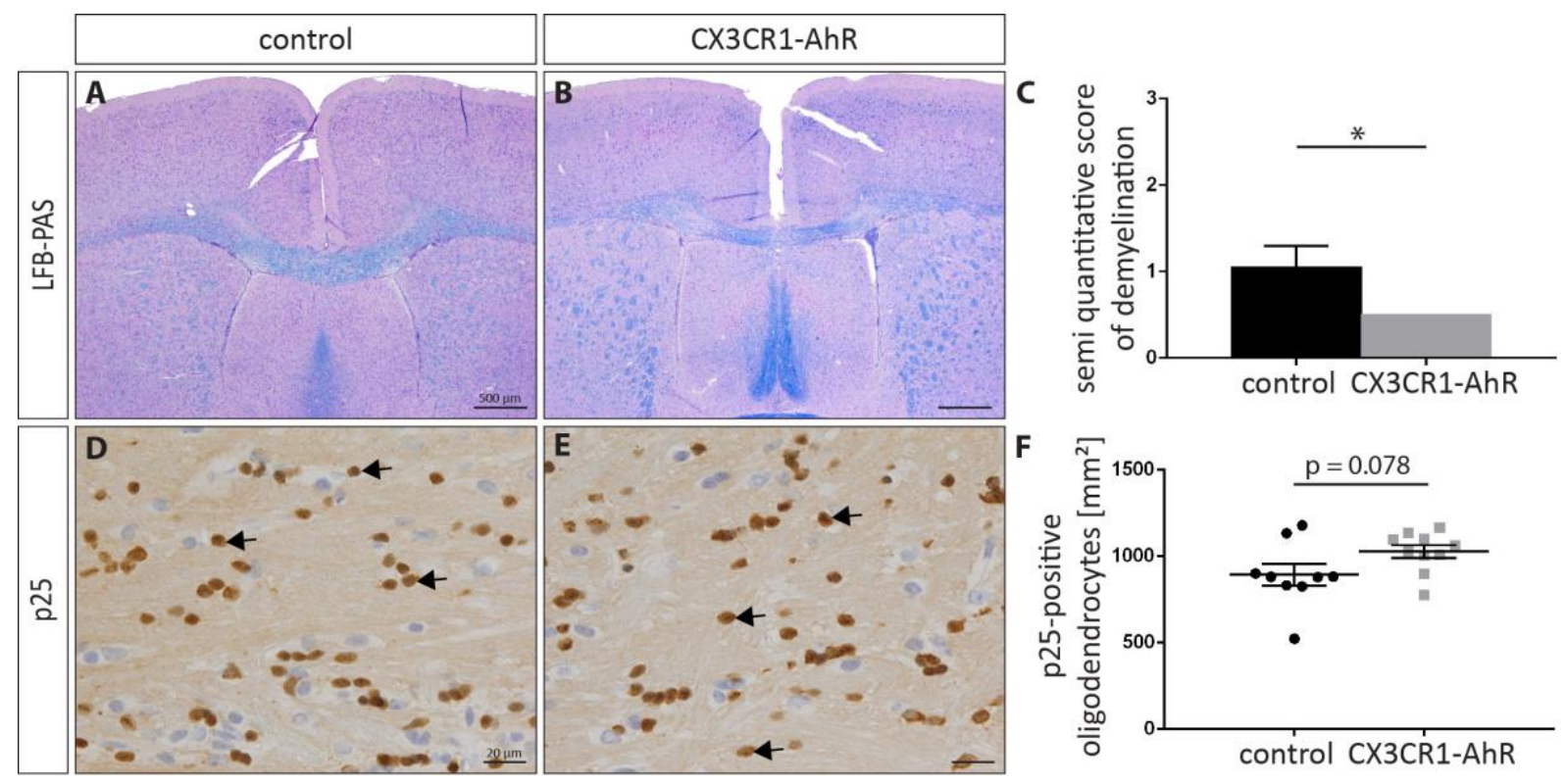

Figure 15: 3 weeks of cuprizone challenge reveals less demyelination and slightly higher numbers of mature oligodendrocytes in CX3CR1-AhR mice compared to control animals

Histological and immunohistochemical evaluation of sections from CX3CR1-AhR $(n=10)$ and littermate control mice $(n=9)$ after 3 weeks of cuprizone feeding. (A, B) LFB-PAS and (D, E) TPPP/p25 IHC of representative sections of corpora callosa from control and CX3CR1-AhR mice, respectively. (C) Quantification of the extent of demyelination by scoring LFB-PAS staining in the entire corpus callosum. A score of 0 indicates complete myelination, a score of 3 demonstrates complete demyelination. (F) Quantification of TPPP/p25 mature oligodendrocytes in the corpus callosum of respective animals. Experiment was conducted and analyzed by Susanne Schmid, while Insa Borgmann performed cell counting (F). LFB-PAS staining data were evaluated by Mann-Whitney test $\left({ }^{*} p<0.05\right)$ (C). TPPP/p25 staining data were analyzed by unpaired $t$ test (F). Data are presented as mean \pm SEM and originate from one experiment. Arrows point to TPPP $/ \mathrm{p} 25^{+}$mature oligodendrocytes (D, E). Scale bars (A, B) $500 \mu \mathrm{m}$ (D, E) $20 \mu \mathrm{m}$.

To analyze whether microglial AhR loss also affects the numbers of mature oligodendrocytes following 3 weeks of toxic demyelination, TPPP/p25-positive cells were counted and evaluated. In comparison with control animals (892.9 \pm 62.9 TPPP/p25 cells per $\mathrm{mm}^{2}$ ), the density of mature oligodendrocytes was mildly elevated in CX3CR1-AhR animals $\left(1027 \pm 37\right.$ TPPP $/ \mathrm{p} 25^{+}$cells per $\left.\mathrm{mm}^{2}\right)$, albeit without significant difference $(p=0.078$, unpaired t test) (Figure $15 \mathrm{D}-\mathrm{F}$ ). 


\subsubsection{No alteration in acute axonal injury but less microglial activation in CX3CR1-AhR mice after 3 weeks of cuprizone treatment}

To elucidate microglial activity in CX3CR1-AhR mice following $0.25 \%$ cuprizone intoxication for 3 weeks, the densities of Mac3+ microglia in the corpora callosa were determined. Compared to littermate controls (580.4 $\pm 85.2 \mathrm{Mac}^{+}$cells per $\mathrm{mm}^{2}$ ), CX3CR1AhR mice displayed significantly less Mac3-positive cells $\left(337.2 \pm 27.1 \mathrm{Mac3}^{+}\right.$cells per $\mathrm{mm}^{2}$; $p=0.011$, unpaired $t$ test with Welch's correction) (Figure $16 \mathrm{~A}-\mathrm{C}$ ).
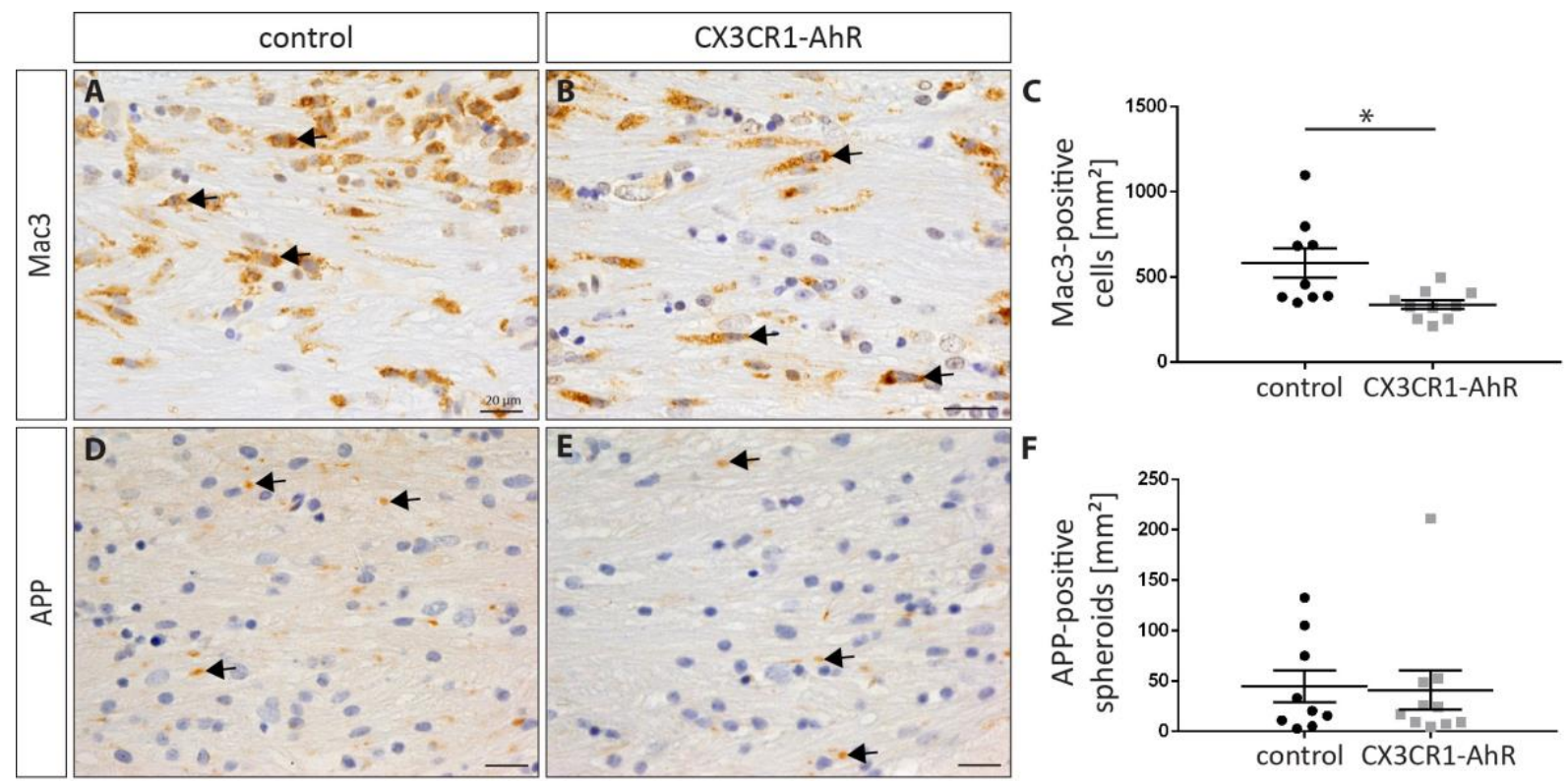

Figure 16: Microglia-specific AhR deletion has no effect on acute axonal injury but decreases microglial activation after 3 weeks of cuprizone intake

(A, B) Mac3 and (D, E) APP IHC of brain sections from CX3CR1-AhR $(n=10)$ and littermate control mice $(n=9)$ 3 weeks after cuprizone treatment. Representative sections are shown. (C) Quantification of $\mathrm{Mac}^{+}$cells for CX3CR1-AhR mice and control mice. (F) Quantification of APP' axonal spheroids in the corpora callosa of respective animals. Data derive from one experiment and are presented as mean \pm SEM. Experiments were conducted and analyzed by Susanne Schmid, while Insa Borgmann performed all cell counting (C, F). Mac3 data were evaluated by unpaired $t$ test with Welch's correction (C), APP staining data were analyzed by Mann-Whitney test. Arrows indicate $\mathrm{Mac}^{+}$activated microglia (A, B) and APP+ axonal spheroids (D, E). Scale bars (A, B, D, E) $20 \mu \mathrm{m}$.

To evaluate the effect of microglial AhR loss on axonal integrity, we measured the frequency of swollen and accumulated APP, indicating acute axonal damage, in both experimental groups. Quantifications resulted in no difference in acutely damaged axons between control animals $\left(44.7 \pm 16.0 \mathrm{APP}^{+}\right.$spheroids per $\left.\mathrm{mm}^{2}\right)$ and CX3CR1-AhR mice $(41.0 \pm$ 19.6 $\mathrm{APP}^{+}$spheroids per $\mathrm{mm}^{2}$, Mann-Whitney test) (Figure $16 \mathrm{D}-\mathrm{F}$ ). 


\subsubsection{Microglial CYP1A1 is increased after FICZ treatment in vitro}

Before measuring CYP1A1 transcript levels in CX3CR1-AhR microglia in further studies, we performed a pilot study to determine general CYP1A1 expression levels in wild type microglia. For this approach, primary mouse microglia were isolated from newborn C57BL/6 mice, purified by magnetic-activated cell sorting and stimulated with FICZ or vehicle (1\% DMSO) for 6 h. QPCR analysis revealed a 5.6-fold upregulation in CYP1A1 mRNA levels after FICZ stimulation $(5.6 \pm 0.4)$ compared to microglia treated with DMSO $(1.0 \pm 0.1)$ (Figure 17). However, this effect did not reach statistical significance.

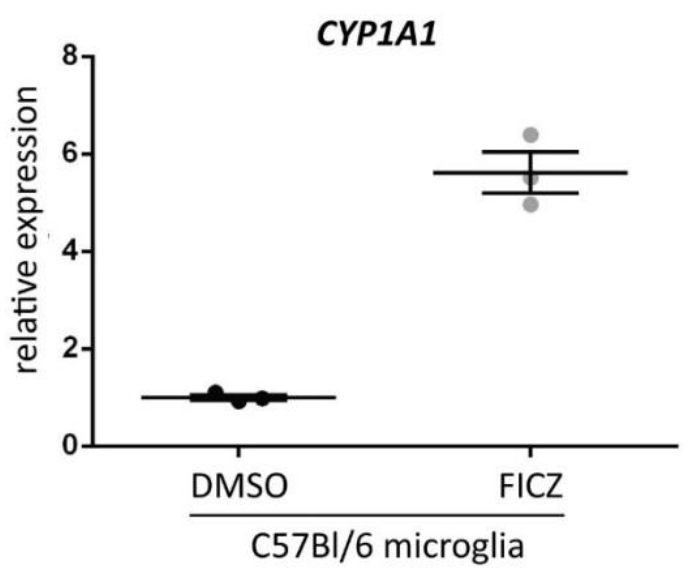

Figure 17: Increased CYP1A1 expression in FICZ-treated control microglia

Quantitative real-time PCR for CYP1A1 expression relative to housekeeping gene GAPDH in RNA isolated from purified microglia from newborn C57BL/6 mice after FICZ treatment. 100,000 cells per well were seeded in triplicates and stimulated with vehicle (1\% DMSO in culture medium) or FICZ (100 nM) for $6 \mathrm{~h}$. Data are presented as mean \pm SEM. Statistical significance was analyzed by Mann-Whitney test. 


\subsection{Part 2: The role of $L A Q$ as AhR ligand during cuprizone-induced demyelination}

\subsubsection{Effects of astrocyte- and microglia-specific AhR deletion on LAQ efficacy in vivo}

\subsubsection{LAQ inhibits cuprizone-induced pathology independent of astrocytic AhR}

Previous studies in our laboratory have demonstrated that laquinimod (LAQ), a quinoline3-carboxamide, prevents oligodendrocyte apoptosis and demyelination after cuprizone intake through reduced astrocytic NF-KB activation (Brück et al. 2012). This immunomodulatory drug, initially developed to treat MS, crosses the blood-brain barrier (BBB) and attenuates experimental autoimmune encephalomyelitis in an AhR-dependent manner (Kaye et al. 2016). Thus, we wanted to evaluate whether the therapeutic effects of LAQ during cuprizoneinduced demyelination are mediated by astrocytic AhR.

For this approach, GFAP-AhR and control mice were fed with $0.25 \%$ cuprizone and received $25 \mathrm{mg} / \mathrm{kg}$ LAQ once daily by oral gavage for 6 weeks. At any time, AhR knockout mice treated with LAQ $(n=8)$ displayed higher body weights than vehicle-treated GFAP-AhR mice ( $n=9$ ) during $0.25 \%$ cuprizone challenge (Figure 18 ).

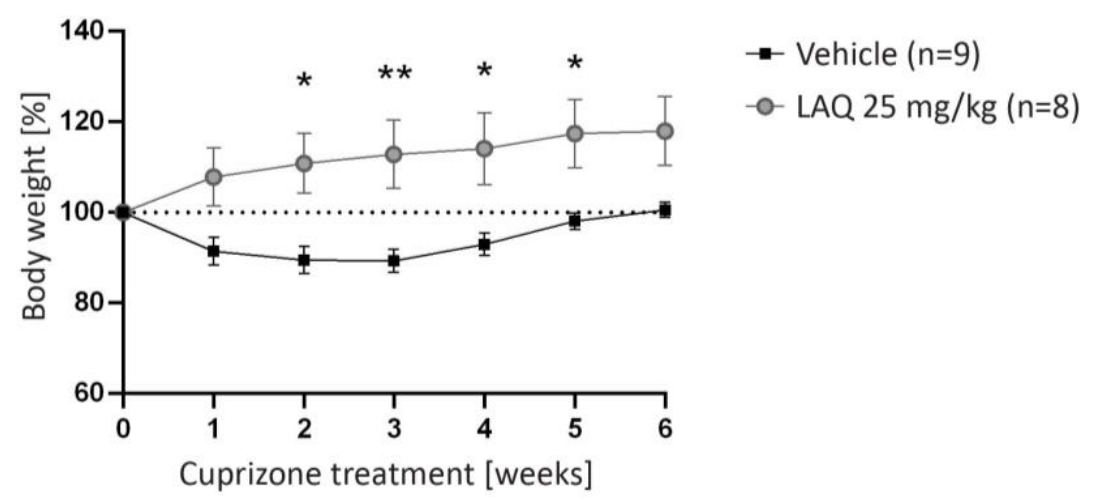

Figure 18: No weight loss in LAQ-treated GFAP-AhR mice after cuprizone exposure

Body weight curves of GFAP-AhR mice treated orally with $25 \mathrm{mg} / \mathrm{kg}$ LAQ $(n=8)$ or vehicle $(n=9)$ relative to their initial weights at experiment start. Animals were fed with a $0.25 \%$ cuprizone diet for 6 weeks. Data originate from 2 independent experiments. Data are expressed as mean values with standard error of the mean (SEM). Statistical significance was tested by two-way ANOVA followed by Sidak's multiple comparisons test. ${ }^{*} p<0.05$, $* * p<0.01$. 
Vehicle-treated mice showed weight loss until week 3, whereas LAQ-treated mice displayed a continuous weight gain. Between weeks 2 and 5 of cuprizone diet, differences in body weights reached statistical significance (weeks 2, 4, 5: $p<0.05$; week 3: $p<0.01$, twoway ANOVA with Sidak's multiple comparisons test) (Figure 18).

Demyelination was evaluated in the corpus callosum for each group. Vehicle-treated animals showed extensive callosal demyelination (Figure $19 \mathrm{~A}, \mathrm{C}$ ), whereas mice treated with LAQ displayed mainly intact callosal myelin (Figure 19 B, D). The demyelination scores were significantly higher in both vehicle groups than in mice treated with LAQ (treatment: $F(1,73)=104.9 ; p<0.0001$, two-way ANOVA). Consequently, we observed no difference between LAQ-treated littermate control and GFAP-AhR mice (genotype: $F(1,73)=0.01937$; $p=0.8897$ ) (Figure $19 \mathrm{E}$ ).

The density of mature oligodendrocytes, assessed by the quantification of TPPP/p25positive cells in the corpus callosum, was significantly higher in both LAQ-treated groups compared to vehicle-treated animals (treatment: $F(1,72)=29.76 ; p<0.0001$ ) (Figure $19 \mathrm{~F}$ J). Thus, there was no difference in the densities of mature oligodendrocytes between LAQtreated AhR knockout and control mice (genotype: $F(1,72)=0.02259 ; p=0.8810$ ).

The density of activated microglia within the corpus callosum was - independent of astrocytic AhR expression - significantly reduced by LAQ treatment compared to vehicle treatment (treatment: $F(1,73)=69.42 ; p<0.0001$; genotype: $F(1,73)=0.08529 ; p=0.7711$ ) (Figure $19 \mathrm{~K}-\mathrm{O})$.

To determine the effect of LAQ on axonal integrity in the absence of astrocytic AhR signaling, acutely damaged axons, characterized by swelling and accumulation of amyloid precursor protein, were evaluated in mice with astrocytic AhR deficiency. There was a significant reduction in acute axonal damage under LAQ treatment in both control and knockout mice compared to both vehicle-treated groups (treatment: $F(1,73)=61.72$; $\mathrm{p}<0.0001$ ) (Figure $19 \mathrm{P}-\mathrm{T}$ ). Thereby, LAQ showed protection against axonal injury regardless of AhR expression in astrocytes (genotype: $F(1,73)=0.07895 ; p=0.7795$ ). 

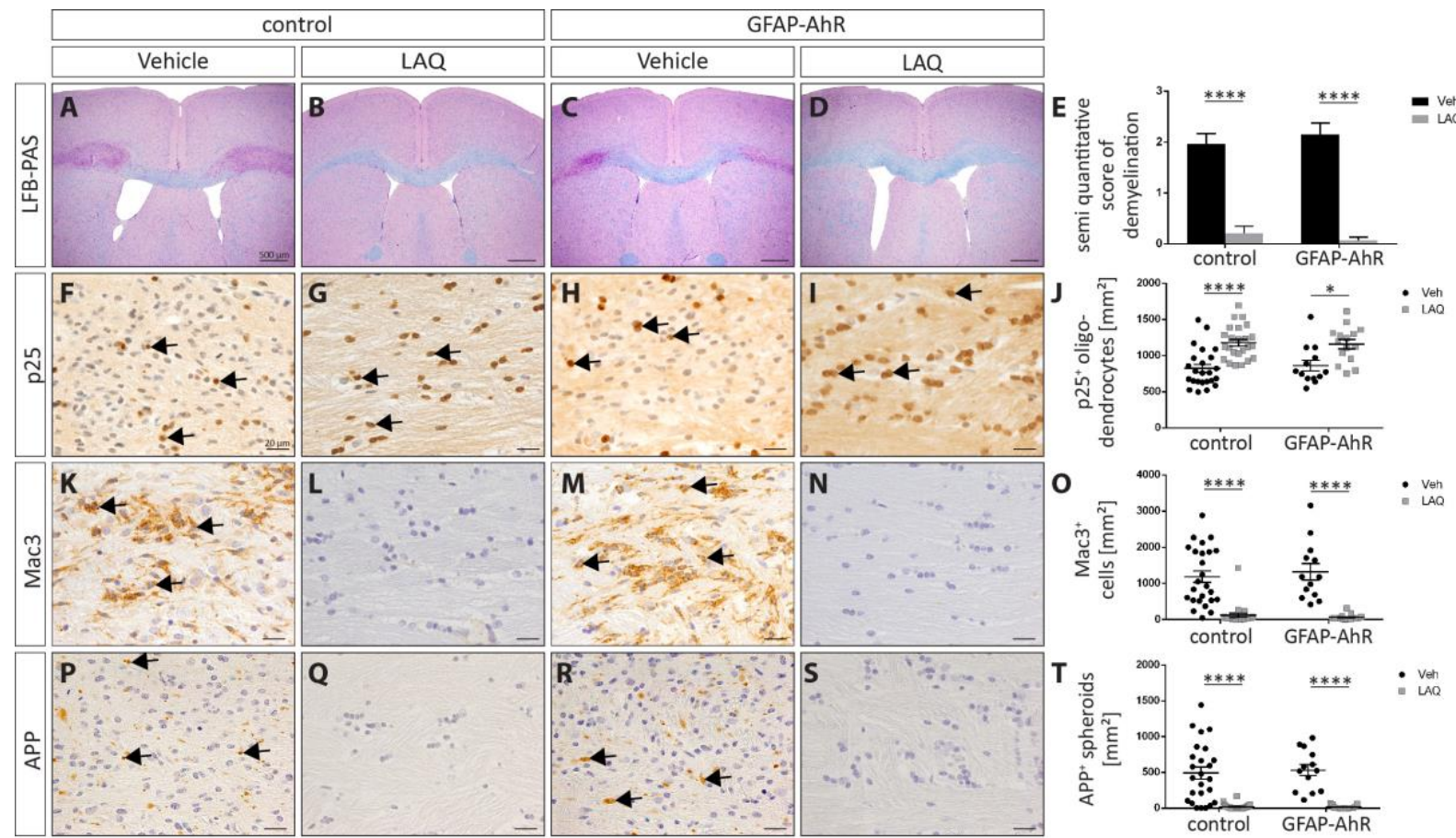

Figure 19: LAQ reduces demyelination, microglial activation, acute axonal damage and loss of mature oligodendrocytes after 6 weeks of cuprizone independent of astrocytic AhR Histological and immunohistochemical evaluation of sections from GFAP-AhR and control littermates. (A-D) LFBPAS histochemistry, (F-I) TPPP/p25, (K-N) Mac3 and (P-S) APP IHC of representative sections of corpora callosa from GFAP-AhR and littermate control mice, respectively, after 6 weeks of cuprizone feeding. (E) Quantification of the extent of demyelination by scoring LFB-PAS staining in the entire corpus callosum. (J) Quantification of $\mathrm{TPPP} /{\mathrm{p} 25^{+}}^{+}$callosal mature oligodendrocytes of respective animals. (0) Quantification of $\mathrm{Mac}^{+}$activated microglia in the corpus callosum. (T) Quantification of APP ${ }^{+}$axonal spheroids. $n$ (control + Veh) $=25 . n$ (control + $\mathrm{LAQ})=24 . n(\mathrm{GFAP}-\mathrm{AhR}+\mathrm{Veh})=13 . n(\mathrm{GFAP}-\mathrm{AhR}+\mathrm{LAQ})=15$. Data are presented as mean $\pm \mathrm{SEM}$ and originate from 2 independent experiments. Arrows point to TPPP $/ \mathrm{p} 25^{+}$mature oligodendrocytes (F-I), activated microglia $(\mathrm{K}, \mathrm{M})$ and axonal spheroids $(\mathrm{P}, \mathrm{R})$. Statistical significance was tested by two-way ANOVA followed by Sidak's multiple comparisons test (E, J, O, T). ${ }^{*} \mathrm{p}<0.05,{ }^{* * * *} \mathrm{p}<0.0001$. Scale bars (A-D) $500 \mu \mathrm{m}$ (F-I, K-N, P-S) $20 \mu \mathrm{m}$.

\subsubsection{LAQ reduces demyelination, microglial activation, acute axonal injury and loss of mature oligodendrocytes independent of AhR deletion in Aldh1l1 $1^{+}$astrocytes}

Using the pan-astroglial marker Aldh1l1, astrocytes can be targeted with high efficiency and specificity (Winchenbach et al. 2016). While GFAP shows preferential expression in white matter, Aldh1l1 is expressed throughout the brain (Cahoy et al. 2008). To assess the effect of LAQ treatment on mice with astrocytic AhR deletion after cuprizone challenge, Aldh1l1-AhR and Cre-negative control mice were pre-treated with tamoxifen at a concentration of $75 \mathrm{mg} / \mathrm{kg}$ on 5 consecutive days to accomplish efficient recombination of AhR in Aldh1l1 ${ }^{+}$ astrocytes. Three days later, toxic demyelination was induced by feeding $0.25 \%$ cuprizone to knockout and control mice. Simultaneously, mice received LAQ orally at a concentration of $25 \mathrm{mg} / \mathrm{kg}$ for 6 weeks. 
Vehicle-treated Aldh1l1-AhR animals showed already significantly reduced body weights after 1, 2 and 3 weeks of $0.25 \%$ cuprizone treatment, whereas Aldh1l1-AhR mice treated with LAQ displayed solely a minimal weight loss after 1 week of cuprizone, followed by constant body weights (weeks 1, 3: $p<0.05$, week 2: $p<0.001$, two-way ANOVA with Sidak's multiple comparisons test) (Figure 20).

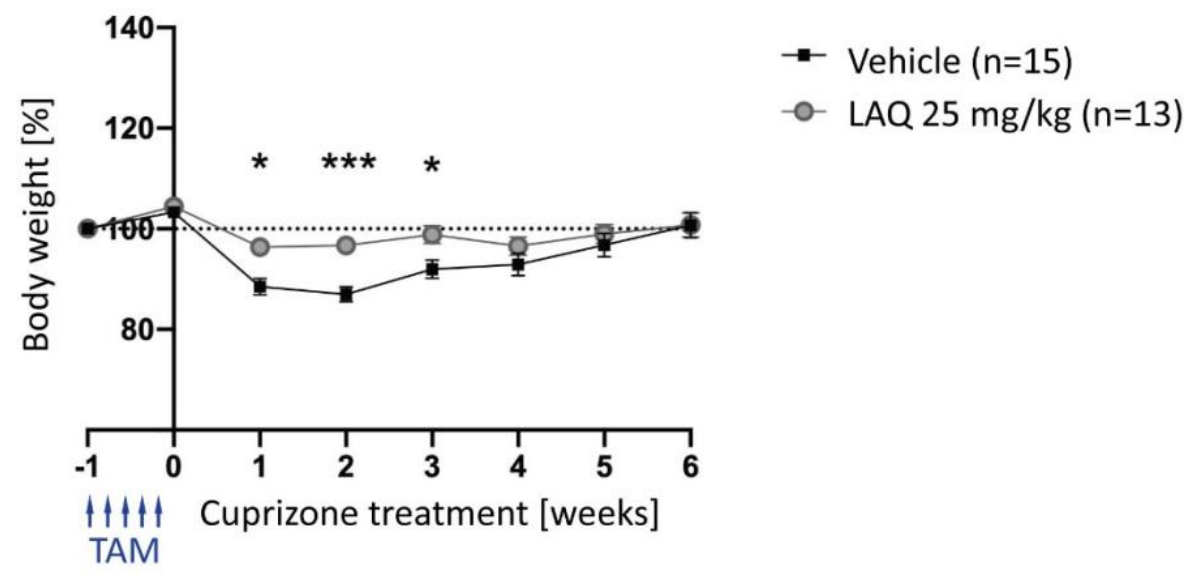

Figure 20: LAQ-treated Aldh1l1-AhR mice show constantly higher body weights than vehicletreated animals during cuprizone-induced demyelination

Body weight curves of Aldh1l1-AhR mice relative to their initial body weights at experiment start. Mice received $25 \mathrm{mg} / \mathrm{kg}$ LAQ $(n=13)$ or vehicle $(n=15)$ by oral gavage once daily and a $0.25 \%$ cuprizone diet for 6 weeks. Animals were pre-treated with tamoxifen intraperitoneally $(75 \mathrm{mg} / \mathrm{kg}$ body weight) on 5 consecutive days one week before experiment start. Data originate from 2 independent experiments. Data are expressed as mean values \pm SEM. Statistical significance was tested by two-way ANOVA followed by Sidak's multiple comparisons test. $* p<0.05, * * * p<0.001$.

In both vehicle-treated Aldh1|1-AhR and control mice, extensive callosal demyelination was observed (Figure $21 \mathrm{~A}, \mathrm{C}$ ), while LAQ-treated mice showed largely intact callosal myelin (Figure $21 \mathrm{~B}, \mathrm{D})$. The demyelination scores were significantly higher in both vehicle-treated groups than in mice treated with LAQ (treatment: $F(1,46)=180.8 ; p<0.0001$, two-way ANOVA). Hence, the protective effects of LAQ on cuprizone-induced demyelination are independent of astrocytic AhR expression (genotype: $F(1,46)=6.087 ; p=0.3009$ ) (Figure $21 \mathrm{E})$. 


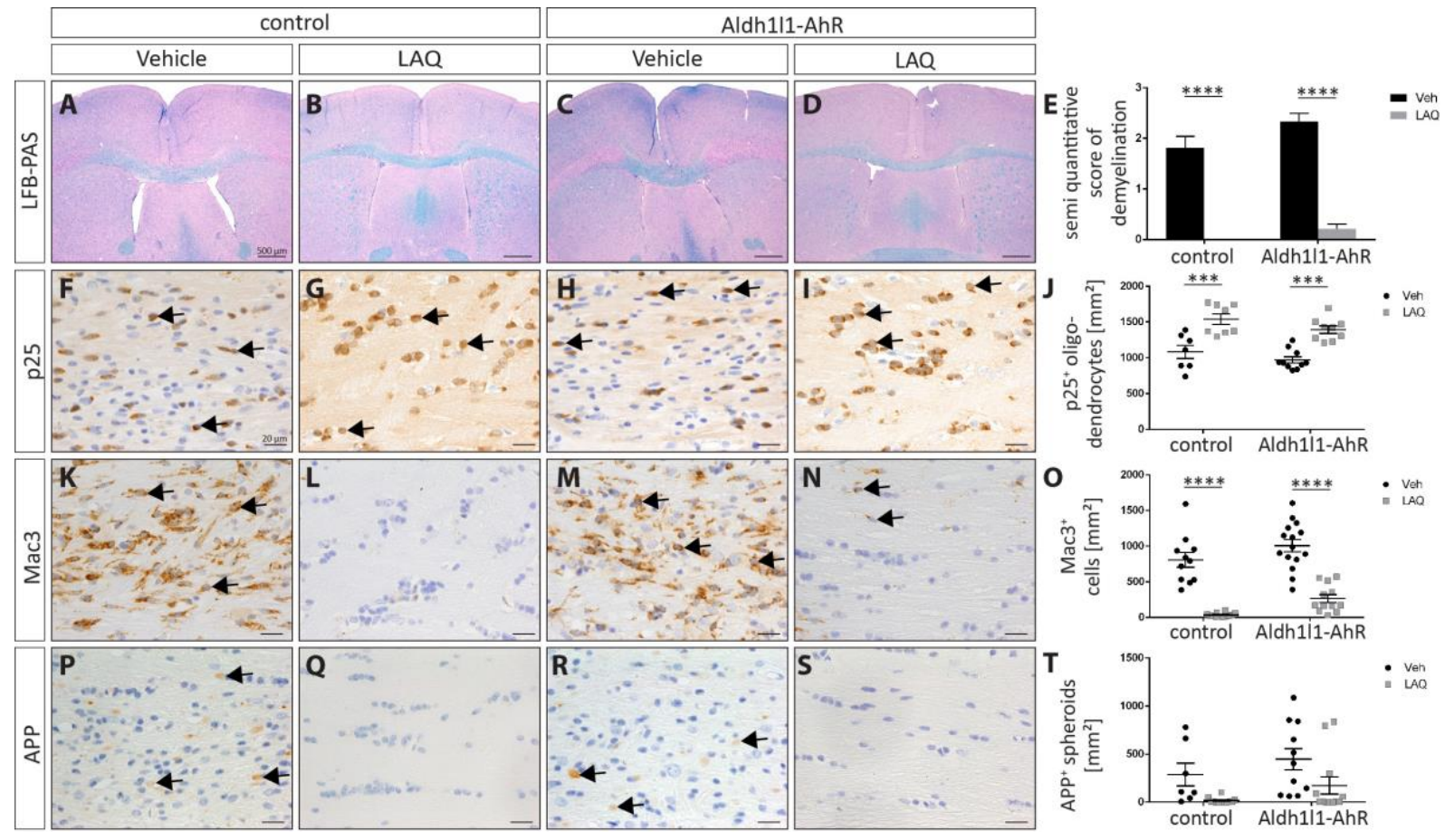

Figure 21: LAQ reduces cuprizone-induced demyelination, microglial infiltration, acute axonal damage and loss of mature oligodendrocytes regardless of AhR deletion in Aldh1l1positive astrocytes

Histological and immunohistochemical evaluation of sections from Aldh1l1-AhR and littermate control mice. All mice were pre-treated with tamoxifen $(75 \mathrm{mg} / \mathrm{kg}$, i.p.) on 5 consecutive days followed by vehicle or LAQ treatment once daily for 6 weeks ( $25 \mathrm{mg} / \mathrm{kg}$, oral gavage). (A-D) LFB-PAS histochemistry, (F-I) TPPP/p25, (K-N) Mac3 and (P-S) APP stainings of representative sections of corpora callosa from Aldh1l1-AhR and littermate control mice, respectively, after 6 weeks of cuprizone diet. (E) Quantification of the extent of demyelination by scoring LFB-PAS staining in the entire corpus callosum. $n$ (control + Veh $)=11 . n$ (control/Aldh1/1-AhR + LAQ $)=$ 12. $n$ (Aldh1l1-AhR + Veh) $=15$. (J) Quantification of TPPP/p25 mature oligodendrocytes in the corpus callosum of respective animals. $n$ (control + Veh $)=7 . n$ (control + LAQ $)=8 . n$ Aldh1l1-AhR + Veh $)=10 . n$ (Aldh1l1-AhR + LAQ) $=9$. (O) Quantification of Mac3 ${ }^{+}$activated microglia in the corpus callosum. $n$ (control + Veh) $=11$. $n$ (control/Aldh1l1-AhR + LAQ $)=12 . n$ (Aldh1l1-AhR + Veh $)=15$. (T) Quantification of APP+ axonal spheroids. $n($ control + Veh $)=7 . n($ control + LAQ $)=13 . n($ Aldh1l1-AhR + Veh $)=11 . n($ Aldh1l1-AhR + LAQ $)=12$. Data are presented as mean \pm SEM and originate from 2 independent experiments. Arrows point to TPPP/p25+ mature oligodendrocytes ( $F-1)$, activated microglia $(K, M, N)$ and axonal spheroids $(P, R)$. Statistical significance was tested by two-way ANOVA followed by Sidak's multiple comparisons test $(\mathrm{E}, \mathrm{J}, \mathrm{O}, \mathrm{T}) .{ }^{* * *} \mathrm{p}<0.001,{ }^{* * * *} \mathrm{p}<0.0001$. Scale bars (A-D) $500 \mu \mathrm{m}$ (F-I, K-N, P-S) $20 \mu \mathrm{m}$.

To investigate the effect of $L A Q$ on mature oligodendrocytes in mice with astrocytic AhR deficiency, we quantified the numbers of TPPP/p25-positive cells. Both littermate controls and Aldh1l1-AhR mice displayed a higher density of mature oligodendrocytes after LAQ treatment compared to vehicle-treated animals (treatment: $F(1,30)=46.38 ; p<0.0001$ ) (Figure $21 \mathrm{~F}$ J). Thus, LAQ therapy significantly reduced loss of mature oligodendrocytes in cuprizonetreated mice, however independent of astrocytic AhR (genotype: $F(1,30)=3.933 ; p=0.0566$ ).

The density of activated microglia within the corpus callosum was - independent of astrocytic AhR - significantly lower under LAQ therapy in relation to vehicle treatment 
(treatment: $F(1,46)=104.0 ; p<0.0001$; genotype: $F(1,46)=8.390 ; p=0.0058)$ (Figure $21 \mathrm{~K}$ - 0).

To analyze a potential astrocytic AhR-dependent effect of LAQ on axonal integrity, acutely damaged axons, characterized by staining of APP, were evaluated in Aldh1l1-AhR mice. Acute axonal damage was slightly less in LAQ- compared to vehicle-treated mice independent of AhR deficiency (treatment: $F(1,38)=9.251 ; p=0.0043$; genotype: $F(1,38)=3.197 ; p=0.0817$ ) (Figure $21 \mathrm{P}-\mathrm{T}$ ). In summary, these findings thus indicate that the beneficial effects of LAQ on cuprizone-induced demyelination are independent of astrocytic AhR.

\subsubsection{LAQ prevents cuprizone-induced pathology independent of microglial AhR}

To address the question whether the effect of LAQ during cuprizone-induced demyelination is mediated by microglial AhR, 9- to 16-week-old CX3CR1-AhR mice and littermate controls received tamoxifen injections at a concentration of $75 \mathrm{mg} / \mathrm{kg}$ on 5 consecutive days to induce recombination. Both groups were fed with $0.25 \%$ cuprizone and simultaneously treated with $25 \mathrm{mg} / \mathrm{kg}$ LAQ or vehicle orally once daily for 6 weeks. Clinical and histological analyses were performed to assess vehicle- and LAQ-treated mice with and without microglial AhR deletion.

In the first week of cuprizone challenge, especially vehicle-treated CX3CR1-AhR mice presented weight loss, which continued until week 2. At that time point, LAQ-treated CX3CR1AhR animals showed significantly higher body weights compared to vehicle-treated mice ( $p<0.0001$, two-way ANOVA with Sidak's multiple comparisons test). With continuous cuprizone intoxication, body weights of LAQ-treated mice stayed constant, whereas vehicletreated animals exhibited continuous weight gain (Figure 22). 


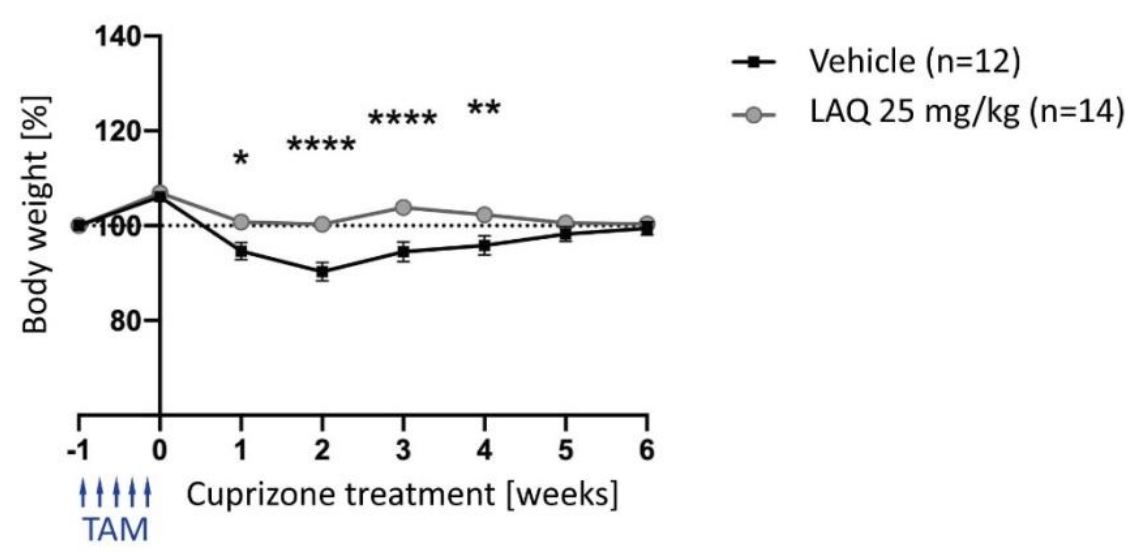

Figure 22: CX3CR1-AhR mice show significantly higher body weights after LAQ treatment compared to vehicle-treated mice during cuprizone intake

Body weight curves of CX3CR1-AhR mice treated with LAQ $(n=14)$ or vehicle $(n=12)$ relative to their initial weights at experiment start one week prior to cuprizone treatment. All mice received intraperitoneal tamoxifen injections ( $75 \mathrm{mg} / \mathrm{kg}$ body weight) on 5 consecutive days. The week after, animals were fed with $0.25 \%$ cuprizone and treated with $\mathrm{LAQ}(25 \mathrm{mg} / \mathrm{kg}$ ) or vehicle (water) once daily by oral gavage for 6 weeks. Animals were at least 9 weeks old at experiment start. Data originate from 2 independent experiments. Data are shown as mean \pm SEM and were analyzed using two-way ANOVA followed by Sidak's multiple comparisons test. ${ }^{*} p<0.05,{ }^{* *} p<0.01$, $* * * * p<0.0001$.

Brain sections of each group were examined and analyzed for demyelination of the corpus callosum using LFB-PAS staining. We could observe significantly lower demyelination scores after LAQ treatment in both littermate control mice and CX3CR1-AhR conditional knockout mice (treatment: $F(1,45)=119.3 ; p<0.0001$, two-way ANOVA) (Figure $23 \mathrm{E}$ ). Both vehicletreated animal groups showed extensive callosal demyelination (Figure $23 \mathrm{~A}, \mathrm{C}$ ), whereas mice treated with LAQ displayed almost completely preserved myelin in the corpus callosum (Figure $23 \mathrm{~B}, \mathrm{D}$ ) independent of microglial AhR (genotype: $F(1,45)=1.255 ; p=0.2685$ ).

Cuprizone-induced demyelination leads to oligodendrocyte apoptosis, accumulation of microglia and acute axonal damage (Irvine and Blakemore 2006; Hiremath et al. 1998; Hesse et al. 2010). Therefore, TPPP/p25, Mac3 and APP immunohistochemistry were used to assess the number of mature oligodendrocytes, activated microglia and to evaluate acute axonal injury. LAQ treatment exhibited protective effects on mature oligodendrocytes of both control and CX3CR1-AhR knockout animals. The number of TPPP/p25-positive oligodendrocytes was significantly higher in LAQ-treated control mice, indicating less cell death, compared to vehicle-treated control mice (Figure $23 \mathrm{~F}, \mathrm{G}, \mathrm{J}$ ). However, the density of mature oligodendrocytes was also higher in LAQ-treated CX3CR1-AhR mice, but this effect did not reach statistical significance when compared to vehicle-treated knockout mice (Figure $23 \mathrm{H}-\mathrm{J}$ ) 
(treatment: $F(1,45)=24.29 ; p<0.0001$; genotype: $F(1,45)=0.6264 ; p=0.4328)$. Thus, LAQ therapy reduced loss of mature oligodendrocytes in cuprizone-treated mice independent of microglial AhR.

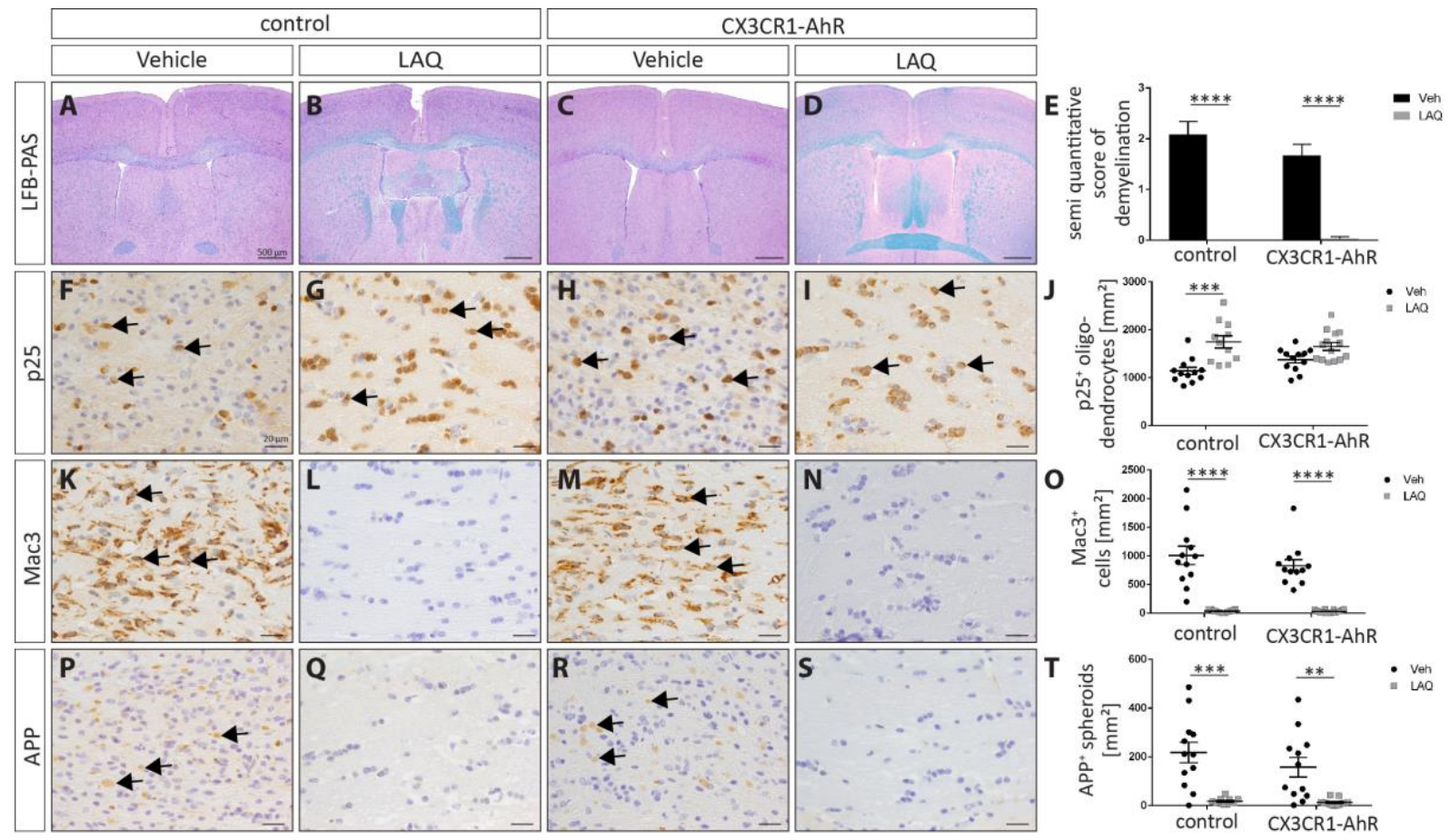

Figure 23: LAQ prevents cuprizone-induced demyelination, microglial activation and acute axonal damage and rescues the density of mature oligodendrocytes independent of microglial AhR

Histological and immunohistochemical evaluation of sections from CX3CR1-AhR and littermate control mice. Mice received tamoxifen intraperitoneally at a concentration of $75 \mathrm{mg} / \mathrm{kg}$ on 5 consecutive days. $72 \mathrm{~h}$ later, animals were treated with vehicle (water) or LAQ $(25 \mathrm{mg} / \mathrm{kg}$ ) once daily by oral gavage and simultaneously fed with $0.25 \%$ cuprizone for 6 weeks. (A-D) LFB-PAS, (F-I) TPPP/p25, (K-N) Mac3 and (P-S) APP stainings of representative sections of corpora callosa. (E) Quantification of the extent of demyelination by scoring LFB-PAS staining in the entire corpus callosum. (J) Quantification of TPPP/p25+ callosal mature oligodendrocytes of respective animals. (0) Quantification of $\mathrm{Mac}^{+}$activated microglia in the corpus callosum. (T) Quantification of $\mathrm{APP}^{+}$axonal spheroids. $n$ (control + Veh $)=12 . n$ (control $\left.+\mathrm{LAQ}\right)=11 . n(\mathrm{CX} 3 \mathrm{CR} 1-\mathrm{AhR}+\mathrm{Veh})=12 . n$ (CX3CR1-AhR $+\mathrm{LAQ})=14$. Data are presented as mean \pm SEM and originate from 2 independent experiments. Arrows point to TPPP/p25 mature oligodendrocytes (F-I), activated microglia (K, M) and axonal spheroids (P, R). Statistical significance was tested by two-way ANOVA followed by Sidak's multiple comparisons test $(E, J, O, T) .{ }^{* *} p<0.01$, ${ }^{* * *} \mathrm{p}<0.001,{ }^{* * * *} \mathrm{p}<0.0001$. Scale bars (A-D) $500 \mu \mathrm{m}$ (F-I, K-N, P-S) $20 \mu \mathrm{m}$.

To determine the effects of LAQ on microglial accumulation during cuprizone-induced demyelination, Mac3-stained sections were evaluated. Both LAQ-treated animal groups showed markedly less activated microglia in the corpus callosum (Figure $23 \mathrm{~L}, \mathrm{~N}$ ) than vehicletreated mice (Figure $23 \mathrm{~K}, \mathrm{M}$ ). Quantitative evaluation supported significantly fewer Mac3positive cells in both control littermates and CX3CR1-AhR mice (treatment: $F(1,45)=87.91$; 
$p<0.0001$; genotype: $F(1,45)=0.9030 ; p=0.3471$ ) (Figure 230 ). Hence, LAQ treatment prevented microglial infiltration under cuprizone, however, independent of microglial AhR.

To analyze the effects of LAQ treatment on axonal integrity, the number of APP-positive spheroids was verified in mice with microglial AhR deficiency and littermate controls during cuprizone intake. The density of APP-positive spheroids was significantly lower in LAQ-treated compared to vehicle-treated controls (treatment: $F(1,44)=34.63 ; p<0.0001$ ) (Figure $23 \mathrm{P}$, $Q, T)$. This LAQ effect was repeated in CX3CR1-AhR mice, indicating no change in acute axonal damage after LAQ treatment between CX3CR1-AhR and control mice (genotype: $F(1,44)=$ $0.1256 ; p=0.2686$ ) (Figure $23 \mathrm{R}, \mathrm{S}, \mathrm{T})$. Thus, these observations suggest that the effect of LAQ on cuprizone-induced demyelination is independent of microglial AhR expression.

\subsubsection{Investigation of $L A Q$ treatment in vitro}

\subsubsection{Astrocytic NF-KB activation is reduced by LAQ independent of AhR}

In previous experimental investigations, we identified astrocytes and microglia cells as the main targets of LAQ in the CNS. We discovered NF-KB downregulation in astrocytes as one of its major mechanisms of action, which led to the decrease of cuprizone-induced demyelination in mice (Brück et al. 2012). However, the effect of LAQ did not involve TLR signaling, which is one of the major pathways leading to downstream NF-KB activation (Kramann et al. 2016). Thus, the exact mechanism of action remains unknown. The AhR limits NF-KB activation via direct interaction with its p65 subunit (Rothhammer and Quintana 2019; Vogel et al. 2014), and the efficacy of LAQ in EAE has been proposed to be AhR-dependent (Kaye et al. 2016). This prompted us to investigate whether the inhibition of NF-KB by LAQ is AhR-dependent. Therefore, we prepared cultures of primary mouse astrocytes from the brains of newborn GFAP-AhR and littermate control mice. A luciferase reporter assay termed Cignal Lenti NF-KB Reporter Kit (SABiosciences) was used to monitor the activity of the NF-KB signaling pathway in astrocytes. Cells were transduced with lentiviral vectors containing a NF-KB-responsive firefly luciferase reporter and a renilla reniformis luciferase normalization reporter. $24 \mathrm{~h}$ after transduction with reporter constructs, cultures were pre-incubated with $250 \mathrm{nM} L A Q$ or vehicle for $2 \mathrm{~h}$ and subsequently treated with a combination of $10 \mathrm{ng} / \mathrm{ml} \mathrm{IL}-1 \beta$ and IFN $\mathrm{f}$ for $1 \mathrm{~h}$. 
Without LAQ, cells exposed to these cytokines displayed significantly increased NF-KB activity in both AhR-deficient and control astrocytes compared to vehicle-treated cells. In contrast to control astrocytes, AhR-deficient astrocytes exhibited significantly decreased NF-KB activity after cytokine stimulation (treatment: $F(2,139)=183.1 ; p<0.0001$ ). However, pre-treatment with $250 \mathrm{nM}$ LAQ resulted in significantly reduced NF-KB activity after cytokine stimulation in both control and AhR-deficient astrocytes (genotype: $F(1,139)=9.754$; $p=0.022)$ (Figure 24).

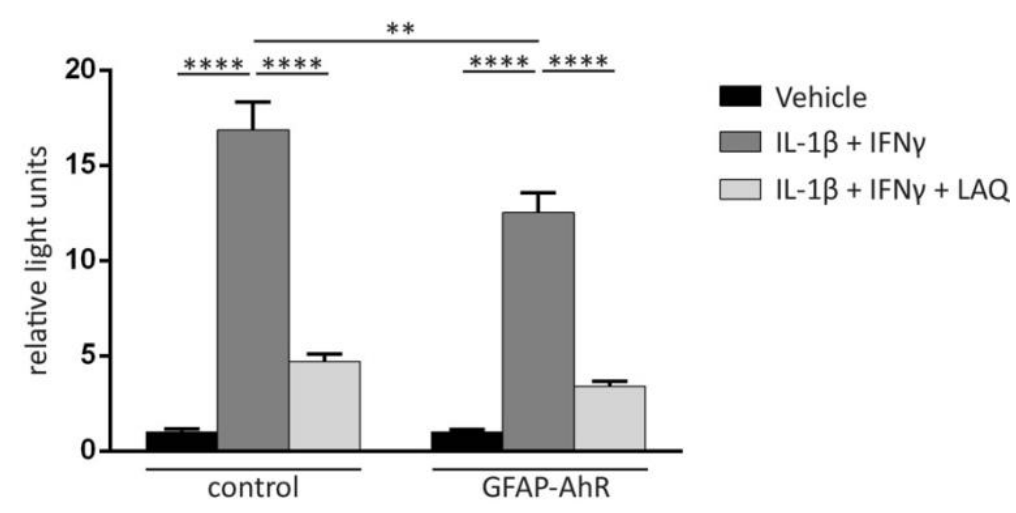

Figure 24: LAQ attenuates astrocytic NF-KB activation independent of astrocytic AhR deficiency

NF-KB reporter gene induction was measured in primary astrocytes from GFAP-AhR $(n=9)$ and littermate control mice $(n=9)$. 100,000 cells were seeded per well as triplicates. Astrocytes were transduced with lentiviral vectors containing NF-KB-responsive firefly luciferase reporter and renilla reniformis luciferase normalization reporter for $24 \mathrm{~h}$. Cells were treated with LAQ $(250 \mathrm{nM})$ or vehicle (water) for $2 \mathrm{~h}$ and subsequently stimulated with a cytokine combination of IL-1 + IFN $\gamma(10 \mathrm{ng} / \mathrm{ml})$ for $1 \mathrm{~h}$. The change in activity of NF-KB was quantified in relative light units and detected by a luminescence plate reader: The more NF-KB was active the more light was produced by firefly luciferase. Graphs show mean values \pm SEM from 2 independent experiments. Statistical significance was tested by two-way ANOVA followed by Sidak's multiple comparisons test. ${ }^{* *} p<0.01,{ }^{* * * *} p<0.0001$.

\subsubsection{LAQ does not induce AhR downstream genes in astrocytes}

In order to evaluate whether changes in mRNA expression of AhR target genes are induced by LAQ treatment, a quantitative PCR study was performed. Murine primary astrocytes from newborn C57BL/6 animals were treated for 6 hours with either $125 \mathrm{nM}$, $250 \mathrm{nM}$ or $500 \mathrm{nM}$ LAQ. $\mathrm{H}_{2} \mathrm{O}$ and DMSO treatment served as negative controls, whereas FICZ treatment at a concentration of $100 \mathrm{nM}$ was used as positive control. Total RNA was isolated and transcribed into cDNA. Subsequently, TaqMan Gene Expression Assay for CYP1A1 was performed. The housekeeping gene GAPDH served as control. 
No difference in CYP1A1 mRNA expression was observed between astrocytes treated with LAQ or vehicle (vehicle treatment: $1.0 \pm 0.2$, LAQ $125 \mathrm{nM}: 0.9 \pm 0.4, \mathrm{LAQ} 250 \mathrm{nM}: 1.0 \pm 0.2$, LAQ $500 \mathrm{nM}: 1.2 \pm 0.3)$. CYP1A1 levels were not upregulated by any concentration of LAQ treatment, while the positive control FICZ resulted in increased CYP1A1 levels by approximately 20-fold (FICZ: $20.0 \pm 2.2$, DMSO: $1.1 \pm 0.2, p<0.05$, Kruskal-Wallis test with Dunn's post-test) (Figure 25).

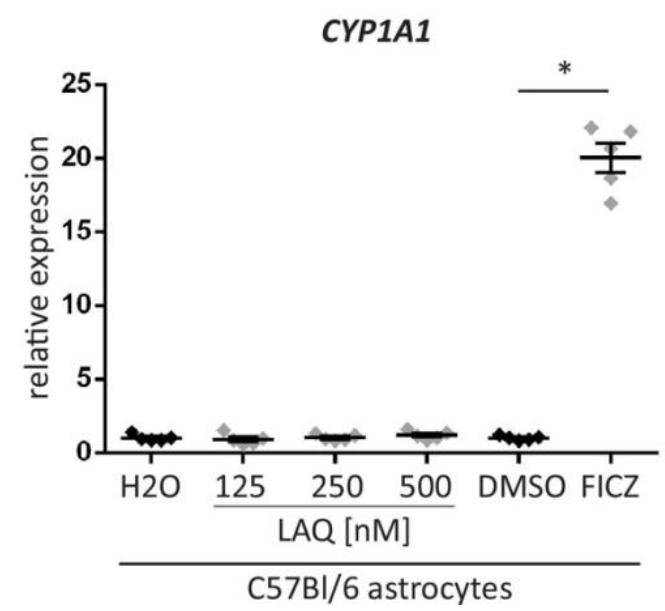

Figure 25: LAQ does not increase CYP1A1 mRNA levels in wild type astrocytes

Transcript levels of CYP1A1 were measured relative to GAPDH using qRT-PCR in RNA isolated from purified astrocytes from newborn C57BL/6 wild type mice $(n=5)$. Cells were treated with LAQ $(125 / 250 / 500 \mathrm{nM})$ or vehicle (water) for $6 \mathrm{~h}$. Treatments with FICZ (100 nM) or vehicle (1\% DMSO) served as controls. Each sample was measured in triplicates. Data are presented as mean \pm SEM. Statistical significance was determined by Kruskal-Wallis test with Dunn's post-test. ${ }^{*} p<0.05$. 


\section{Discussion}

The aryl hydrocarbon receptor (AhR) is a ligand-activated transcription factor which mediates not only the toxicity of xenobiotics but also regulates immune responses (Stockinger et al. 2014). Depending on the ligand, the AhR controls diverse biological processes through ligand-specific, cell type-specific and context-specific mechanisms (Rothhammer and Quintana 2019). Its broad distribution in terms of expression in the body ranges from cells of the innate and adaptive immune system to cells of the central nervous system (CNS). Within the CNS, the AhR was found to act as a negative regulator of astrocytic and microglial nuclear factor-KB (NF-KB) activation. The lack of AhR in glial cells was demonstrated to result in the worsening of experimental autoimmune encephalomyelitis (EAE) (Rothhammer et al. 2016; Rothhammer et al. 2018). Laquinimod (LAQ), originally developed to treat MS, is a small molecule that crosses the blood-brain barrier (BBB) and attenuates EAE by activating the AhR (Brück and Wegner 2011; Kaye et al. 2016). In previous studies, we demonstrated that LAQ protects mice from cuprizone-induced demyelination by attenuation of astrocytic NF-KB activity (Brück et al. 2012). Thus, we hypothesized that the AhR plays an important role in CNS demyelination and can be activated by the presumed and clinically relevant AhR agonist LAQ.

The present work evaluated the role of astrocytic and microglial AhR in toxic demyelination and elucidated its role in mediating the therapeutic effects of LAQ on cuprizone-induced pathology in mice. Expression levels of the AhR target gene CYP1A1 were first measured to confirm cell-specific deletion of AhR. AhR-deficient astrocytes isolated from newborn GFAP- and Aldh1l1-AhR mice showed strongly reduced mRNA levels of CYP1A1 upon stimulation with the AhR ligand FICZ relative to control treatment, supporting AhR depletion. The main aim of this study was to investigate the impact of AhR loss in astrocytes and microglia on cuprizone intoxication. While GFAP-AhR and Aldh1l1-AhR mice showed numbers of mature oligodendrocytes comparable to littermate controls after 6 weeks of cuprizone feeding, CX3CR1-AhR mice presented significantly higher densities of oligodendrocytes. However, demyelination, microglial activation and axonal damage were unaltered in all mouse strains compared to Cre-negative controls.

This work also aimed at assessing whether the therapeutic efficacy of the putative AhR ligand LAQ is mediated via glial AhR signaling in toxic demyelination. LAQ treatment of 
cuprizone-fed mice showed inhibition of mature oligodendrocyte apoptosis, demyelination, microglial infiltration and acute axonal injury in all three mouse strains independent of genotype. Using a reporter-based in vitro assay, we observed significantly reduced NF-KB activation in AhR-deleted primary astrocytes compared to controls. After LAQ treatment, primary astrocytes demonstrated diminished astrocytic NF-KB activation independent of AhR signaling.

\subsection{The AhR is expressed by astrocytes and microglia and upregulated in CNS inflammation}

The localization and diverse essential features of astrocytes and microglia predestine these cells to be prime candidates for sensing and coordinating the response to a variety of endogenous, environmental, metabolic and microbial stimuli, including AhR agonists. The AhR is a ligand-activated transcription factor that participates in a broad array of physiological and pathological processes (Rothhammer and Quintana 2019) due to its far-reaching expression in all types of tissues and cells (Wang et al. 2020). Initially identified as the receptor mediating the metabolism and detoxification of environmental pollutants (Denison and Nagy 2003; Denison et al. 2002), the activation of AhR by ligands derived from the diet, gut flora and the host metabolism has important physiologic functions, including the modulation of the immune response (Quintana and Sherr 2013). The AhR plays a crucial role in the regulation of autoimmune inflammatory diseases of the gut (e.g. Crohn's disease and ulcerative colitis), skin (psoriasis) and the central nervous system (MS) (Colonna 2014; Monteleone et al. 2011; Rothhammer 2016).

During EAE, it was demonstrated that astrocyte-specific deletion of AhR leads to disease worsening and failure to recover. These effects were accompanied by enhanced production of pro-inflammatory mediators, which reflects the increased activation of NF-kB. Notably, AhR agonists provided by the commensal flora or the diet enter the CNS and induce an antiinflammatory response in astrocytes, leading to reduced CNS inflammation (Rothhammer 2016). Apart from astrocytes, it was shown that AhR also inhibits pro-inflammatory transcriptional responses in microglia during EAE (Rothhammer et al. 2018). In both astrocytes and microglia, AhR can limit the activation of NF-KB (Rothhammer 2016; Rothhammer et al. 2018). In brain samples from MS patients, AhR expression was observed to be upregulated, 
which matches the induction of the IFN-I pathway in astrocytes. AhR activation was found to be reduced in CNS lesions compared to controls, and circulating AhR agonists were decreased (Rothhammer 2016). However, the role of AhR signaling in glial cells is still poorly understood (Rothhammer and Quintana 2019). Furthermore, little is known about the relationship between glial AhR and oligodendrocyte death and demyelination occurring in the absence of an adaptive immune response. This led to the hypothesis that a cell-specific, microglial or astrocytic deletion of the AhR may affect the extent and characteristic of CNS pathology in cuprizone-induced demyelination.

\subsection{AhR loss on astrocytes or microglia does not modify cuprizone-induced demyelination}

To evaluate the effects of glia-specific AhR deletion on demyelination, we utilized the cuprizone mouse model which is based on feeding mice a diet containing the copper chelator cuprizone that leads to toxic demyelination in white and grey matter (Gudi et al. 2014). Cuprizone is classically administered for 4 to 6 weeks, which induces oligodendrocyte apoptosis with subsequent significant and reproducible demyelination in diverse CNS structures, including the corpus callosum. One of its hallmarks is an intact BBB. Thus, the usage of this experimental MS model for lesion induction - in contrast to MS and EAE - presents a valuable tool for studying CNS-intrinsic effects and basic cellular and molecular mechanisms during demyelination without the involvement of peripheral immune cells (Gudi et al. 2014; Matsushima 2001; Gudi et al. 2009; Skripuletz et al. 2008; Silvestroff et al. 2010).

GFAP-, Aldh1l1-, CX3CR1-AhR knockout animals and respective Cre-negative littermate controls were fed cuprizone for 6 weeks and demyelination was subsequently analyzed in the corpus callosum. Overall, mice with astrocytic and microglial AhR deletion displayed no differences in the extent of demyelination, number of mature oligodendrocytes, microglial infiltration and acutely damaged axons compared to littermate control animals.

In our conditional knockout mice, AhR is deleted under control of the GFAP, Aldh1l1 or CX3CR1 promoter. GFAP shows preferential expression in white matter over grey matter astrocytes with significantly higher levels in the spinal cord compared to astrocytes in the brain (Cahoy et al. 2008; Yoon et al. 2017). GFAP expression was additionally found in multipotent radial neural stem cells, while astrocyte-related cells outside the brain such as satellite cells in 
peripheral ganglia, enteric glia, non-myelinating Schwann cells or mesenchymal stellate cells in liver, kidney, pancreas, lungs, and testis, also show GFAP expression (Sofroniew and Vinters 2010; Lim et al. 2008; Bush et al. 1998; Jessen et al. 1990; Zhao and Burt 2007; Buniatian et al. 1998; Regoli et al. 2000; Davidoff et al. 2002; Stephenson and Byers 1995). The aldehyde dehydrogenase-1 family member L1 (Aldh1l1), however, was found to be homogenously and specifically expressed in astrocytes within the brain. Compared to GFAP, Aldh1l1 mRNA showed a considerably broader expression pattern throughout the CNS (Cahoy et al. 2008; Winchenbach et al. 2016). Outside the CNS, Aldh1|1-CreERT2 expression was observed in several peripheral organs such as liver, lung, small intestine and kidney (Winchenbach et al. 2016; Krupenko and Oleinik 2002).

Mean demyelination scores from GFAP-, Aldh1l1- and CX3CR1-AhR littermate controls were comparable to each other, in accordance with the same genetic background. Although mice with microglial AhR deficiency showed significantly less demyelination at 3 weeks of intoxication compared to controls, this difference disappeared after continuation of cuprizone treatment for another 3 weeks. Additionally, both CX3CR1-AhR and Cre-negative control mice displayed only incomplete demyelination at 3 weeks of cuprizone feeding compared to a longer treatment duration of 5 to 6 weeks, well in line with the literature (Gudi et al. 2009). After 6 weeks of cuprizone administration, we observed an overall moderate extent of demyelination in the corpus callosum, which is in accordance with results published by Skripuletz and co-workers. Even though the degree of demyelination in the corpus callosum culminates in the sixth week of cuprizone feeding, complete demyelination is usually only reached in the cortex (Skripuletz et al. 2008; Vega-Riquer et al. 2019). This can in part be explained by spontaneous remyelination starting already 3 weeks after cuprizone application (Praet et al. 2014).

Immunohistochemical analyses were performed to evaluate the numbers of mature oligodendrocytes in mice lacking astrocytic and microglial AhR after 6 weeks of cuprizoneinduced demyelination. Based on p25/TPPP, a marker for mature oligodendrocytes, comparable numbers of callosal mature oligodendrocytes were observed in mice lacking astrocytic AhR and Cre-negative controls. This indicates that the density of mature oligodendrocytes after 6 weeks of cuprizone challenge is independent of astrocytic AhR 
deletion. In contrast, mice with microglial AhR deficiency displayed a significantly higher density of mature oligodendrocytes after cuprizone intoxication compared to control animals. These findings are in line with the slightly lesser extent of demyelination we observed in CX3CR1-AhR mice after 6 weeks of cuprizone feeding. Considering the pathophysiology of the cuprizone mouse model, which is based on oligodendrocyte death as an early manifestation during the first 3 weeks of toxic demyelination (Hesse et al. 2010), it would be worthwhile to investigate oligodendroglial apoptosis in these mice to determine whether less mature oligodendrocytes die or whether they repopulate faster. Already after one week of cuprizone administration, dying oligodendrocytes can be detected by expression of activated caspase-3 (Hesse et al. 2010). However, the exact mechanism of oligodendrocyte death in response to cuprizone remains unknown. While microglia appear to be involved in the induction of oligodendrocyte death and demyelination by the production and secretion of proinflammatory cytokines, cuprizone is assumed to be selectively toxic to mature oligodendrocytes by causing mitochondrial malfunctions, subsequently leading to demyelination (Benardais et al. 2013; Matsushima 2001; Pasquini et al. 2007). Since mature oligodendrocytes are preferentially affected, oligodendrocyte precursor cells (OPCs) are activated within the lesion already during active demyelination. In week 3 of cuprizone exposure, OPCs begin to proliferate, differentiate and start to remyelinate, thus initiating a regenerative response (Mason et al. 2000). This explains the incomplete demyelination and relatively high number of mature oligodendrocytes observed in GFAP-, Aldh1l1- and CX3CR1AhR mice - however largely independent of AhR expression - after 6 weeks of cuprizone intoxication.

Taken together, our findings confirm that the cuprizone mouse model constitutes a highly useful, reproducible and non-invasive tool for studying T cell-independent demyelination. However, I conclude from my experiments that AhR deficiency in astrocytes or microglia does not affect cuprizone-induced demyelination and its subsequent consequences, including axonal damage and microglial infiltration.

The results from the present experiments raised the following questions: Why is it that astrocyte- or microglia-specific AhR deletion does not induce substantial changes in cuprizoneinduced pathology while it does so during autoimmune inflammatory demyelination? Are 
there other factors expressed by astrocytes and microglia modulating cuprizone-induced demyelination?

\subsection{Astrocytic NF-KB activation is reduced by LAQ independent of AhR}

Astrocytes are known to play an active and multifaceted role in regulating demyelinating CNS diseases (Nair et al. 2008). In previous studies, we demonstrated that LAQ prevents cuprizone-induced demyelination and down-modulates the pro-inflammatory response in astrocytes by interfering with astrocytic, but not microglial, NF-KB activation (Brück et al. 2012). This effect, however, is independent of upstream toll-like receptor signaling (Kramann et al. 2016).

To address the question whether AhR signaling is essential for downstream activation of astrocytic NF-KB, we studied the effect of LAQ treatment in primary mouse astrocytes lacking the AhR.

The NF-KB family of inducible transcription factors is a critical and rapid regulator of cellular, immune and inflammatory responses (Mc Guire et al. 2013). NF-kB signaling in glial cells has been observed to play a major role in oligodendrocyte damage and demyelination under cuprizone. Mice lacking astrocytic NF-KB activation by conditional ablation of IkB kinase 2 (IKK2) in astrocytes, but not in oligodendrocytes, are protected from myelin loss, show less gliosis and reduced expression of pro-inflammatory mediators during toxic demyelination (Raasch et al. 2011). Remarkably, the NF-KB subunit RelA (p65) was reported to be a crucial regulator of AhR expression and induction of AhR-dependent gene expression in immune cells, indicating a cross-talk between NF-KB and AhR signaling (Vogel et al. 2014; Vogel and Matsumura 2009).

In our study, AhR-deficient astrocytes stimulated with IL-1 $\beta$ and IFN $\gamma$ displayed a strong induction in NF-KB activity compared to unstimulated cells, albeit to a much lesser degree than wild type astrocytes. On the one hand, AhR signaling has been suggested to inhibit NF-KB activation in astrocytes (Rothhammer et al. 2016). This indicates that AhR loss may cause increased NF-KB activity, which thus contradicts our results. On the other hand, LPS-activated NF-KB signaling, which resembles our stimulation with IL-1 $\beta$ and IFN $\gamma$, was shown to enhance AhR expression (Vogel et al. 2014). Thus, another hypothesis might be that the lack or reduced 
expression of AhR equally leads to decreased but persisting NF-KB activity due to cytokine stimulation. Compared to vehicle treatment, application of LAQ still significantly reduced NF-KB activity in astrocytes of GFAP-AhR knockout mice. It is conceivable that other types of astrocytes or further glial cells such as microglia and oligodendrocytes, which are AhR competent, remained in the culture, hence causing the significant response upon LAQ administration. Ultimately, however, it is likely that LAQ attenuates astrocytic NF-KB activation independently of AhR ligation, which is well in line with results obtained in C57BL/6 astrocytes by Brück et al. (Brück et al. 2012).

By the quantification of nuclear translocation of $p 65$, similar results were found in wild type astrocytes after cuprizone intake and LAQ treatment in vivo, showing a diminished astrocytic NF-KB activity (Brück et al. 2012). It is noteworthy that the efficacy of LAQ in CNS autoimmunity, i.e. experimental autoimmune encephalomyelitis, is dependent on the AhR (Kaye et al. 2016; Berg et al. 2016; Rothhammer 2016). Whether LAQ treatment alleviates CNS demyelination in an AhR-dependent manner, also in the absence of peripheral immune cells, has not been studied before. Therefore, we investigated the role of LAQ treatment during toxic demyelination and, more specifically, whether the therapeutic efficacy of the putative AhR ligand LAQ in cuprizone-induced demyelination is mediated via glial AhR signaling in astrocytes or microglia.

After 6 weeks of cuprizone challenge, GFAP-, Aldh1l1- and CX3CR1-AhR mice demonstrated significantly higher body weights after LAQ treatment compared to vehicletreated animals. In line with its beneficial effects on C57BL/6 mice (Brück et al. 2012), LAQ was shown in the present study to protect mice lacking astrocytic or microglial AhR from cuprizone-induced demyelination, microglial infiltration, axonal damage and loss of mature oligodendrocytes.

LAQ inhibits clinical symptoms and ameliorates the disease course by pronounced changes in CNS-resident cell populations, especially by activating an anti-inflammatory response in astrocytes. In both the inflammatory mouse model EAE and the toxic model cuprizone, LAQ was also reported to suppress microglial activation (Nyamoya et al. 2019; Mishra et al. 2014). LAQ reduces microglia-induced neuronal death in vitro and axonal injury/loss in vivo (Mishra et al. 2014). 
Thus, several studies have pointed out that LAQ is protective in CNS-intrinsic cells, but these effects do not involve upstream toll-like receptor signaling (Kramann et al. 2016) or AhR expression on astrocytes or microglia. Based on these findings, I questioned whether other factors expressed by glia cells induced a modulation of cuprizone-induced demyelination.

\subsection{The role and function of astrocytes during cuprizone-induced demyelination}

Astrocytes were shown to modulate demyelination by expression and production of proand anti-inflammatory cytokines and chemokines. Both beneficial and detrimental functions have been suggested (Nair et al. 2008). In response to cuprizone treatment, astrocytes become activated and form an astrogliosis (Sofroniew and Vinters 2010; Sofroniew 2009; Sofroniew 2014), a process which includes hypertrophy of cellular processes, proliferation and enhanced GFAP expression (Pekny and Nilsson 2005; Carlton 1967; Hiremath et al. 1998).

As the main regulator of oxidative homeostasis in the brain, dysfunctional astrocytes are thought to be involved in the accumulation of oxidative damage. In a study by Draheim and colleagues, hyperactivation of astrocytic Nrf2-signaling was induced in mice by astrocytespecific deletion of the Nrf2 repressor Keap1. Interestingly, hyperactivation of Nrf2 as the main transcriptional regulator of the anti-oxidant stress defense was sufficient to impede cuprizone-induced oligodendrocyte loss, microgliosis and axonal damage (Draheim et al. 2016).

GFAP expression plays an important role in astrocyte signaling. Only recently, we found that overexpression of GFAP inhibits CXCL10 production and NF-KB activation. As a consequence, mice were protected from cuprizone-induced demyelination, oligodendrocyte apoptosis, microglial infiltration and acute axonal damage (Kramann et al. 2019). In the present study, mice lacking AhR in GFAP-positive astrocytes displayed no change in cuprizoneinduced pathology after 6 weeks of toxic demyelination. This is in accordance with findings by Kriszta et al. who investigated mGFAP-driven conditional deletion of the TRPA1 receptors, which are non-selective cation channels controlling resting $\mathrm{Ca}^{2+}$ levels and inhibitory synapses (Kriszta et al. 2019; Shigetomi et al. 2011). While no significant differences could be observed after 6 weeks, they detected significantly less demyelination between the $3^{\text {rd }}$ and $5^{\text {th }}$ week of 
cuprizone treatment. Based on these results, the AhR on astrocytes may transiently contribute to the demyelination induced by cuprizone.

Besides their deleterious role in CNS lesions, astrocytes may also lessen inflammation and support neuroprotection and lesion repair. Brain-derived neurotrophic factor (BDNF) is a neurotrophin expressed by astrocytes and neurons (Lee et al. 2012). Elevated astrocytederived synthesis of BDNF, initiated by glutamate metabotropic stimulation, has been shown to promote the production of myelin proteins in the lesioned corpus callosum and to result in the recovery from cuprizone-elicited demyelination (Fulmer et al. 2014).

During cuprizone-induced demyelination, a crucial crosstalk between astrocytes and microglia has been identified (Gudi et al. 2014). In EAE, mice with astrocyte-specific deletion of CCL2 demonstrated an ameliorated disease course with reduced immune cell response, activation of astrocytes and microglia (Kim et al. 2014). In the cuprizone model, however, astrocytes were shown to produce the chemokine CXCL10, which induces early recruitment of microglia to demyelinating lesions for the phagocytosis of myelin debris. In addition, genetic ablation of reactive astrocytes did not protect mice from cuprizone-induced myelin damage, loss of oligodendrocytes and axonal injury. Instead, depletion of astrocytes led to reduced microglial activation and inhibited clearance of myelin debris, followed by impaired OPC proliferation and remyelination (Skripuletz et al. 2013).

\subsection{The role and function of microglia under cuprizone}

In the intact brain, microglia are responsible for immune surveillance and constantly scan the parenchyma for injuries to neurons, oligodendrocytes or astrocytes (Nimmerjahn et al. 2005). These "resting" microglia show a branched morphology and dynamically extend and retract their highly ramified processes (Davalos et al. 2005; Nimmerjahn et al. 2005; Wake et al. 2009). It is noteworthy that microglia are known to play a crucial role in demyelinating lesions in the CNS. Upon CNS injury, they become activated and transform into an amoeboid shape by retracting their processes (Kettenmann et al. 2011).

In the cuprizone mouse model, activated microglia are recruited to the sites of demyelination such as the corpus callosum, where they proliferate (Remington et al. 2007) and substantially increase in number, which is termed microgliosis (Hiremath et al. 1998; Li 
and Zhang 2016). As the resident macrophages of the CNS, microglia have important functions that can be both beneficial and detrimental. Regarded as a driving factor behind apoptosis of metabolically stressed oligodendrocytes, microglia are involved in efficient clearance of myelin debris, damaged cells or microbes following cuprizone challenge (Pasquini et al. 2007; Praet et al. 2014; Colonna and Butovsky 2017; Kettenmann et al. 2011).

Microglia were shown to play a key role in the maintenance of OPC numbers and myelinogenesis (Hagemeyer et al. 2017). In mice lacking colony-stimulating factor 1 receptor (CSF1R) on microglia, which is vital for microglial proliferation and survival (Elmore et al. 2014), cuprizone-induced microgliosis was significantly decreased in corpus callosum (Pons et al. 2020). Simultaneously, the production of OPCs was reduced and the removal of myelin debris was negatively affected (Laflamme et al. 2018). A study conducted by Lampron and colleagues supports the crucial role of microglia in phagocytosis of damaged myelin, showing that the clearance of myelin debris was severely impaired in CX3CR1-deficient mice. Thus, animals displayed limited cuprizone-induced demyelination and microglial infiltration, while oligodendroglial survival was not enhanced (Lampron et al. 2015).

Similarly, we found that mice devoid of the AhR in CX3CR1-positive microglia showed a significant reduction in microglial activation at 3 weeks of intoxication compared to controls, which correlated with less demyelination. However, these differences were no longer present after 6 weeks of cuprizone treatment. Additionally, mice displayed - independent of microglial AhR - a strong increase in phagocyte activation from 3 to 6 weeks of cuprizone feeding, which is in accordance with previous studies (Gudi et al. 2009). Collectively, our data suggest that glial AhR may transiently be involved in cuprizone-induced pathology.

Compared to CX3CR1, Sall1 was described to be an essential transcriptional regulator that specifically targets microglia but not other tissue macrophages and myeloid cells. Moreover, tamoxifen treatment of Sall1 ${ }^{\mathrm{CreER}} \mathrm{Csf1} \mathrm{r}^{\mathrm{fl} / \mathrm{fl}}$ mice or treatment with the CSF1R inhibitor PLX5622 of Sall $1^{\mathrm{GFP} /+}$ mice resulted in rapid depletion of microglia, while Sall $1^{\mathrm{CreER}} \mathrm{Tgfbr}^{\mathrm{fl} / \mathrm{fl}}$ mice showed modified cell morphology, indicating that CSF1R and TGF- $\beta R$ are important factors for microglial homeostasis (Buttgereit et al. 2016; Butovsky et al. 2014). After depletion, microglia were completely repopulated within 7 days by the residual CNS-resident microglia (Buttgereit et al. 2016; Waisman et al. 2015). In one of several other depletion systems, microglia from

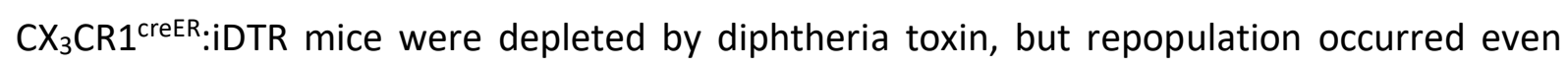


after 5 days (Bruttger et al. 2015; Parkhurst et al. 2013; Waisman et al. 2015). During cuprizone challenge, prophylactic treatment with the CSF1R kinase inhibitor BLZ945, which induces microglia ablation, leads to increased myelin debris and axonal pathology in the cortex and external capsule but inhibited callosal demyelination, while therapeutic treatment resulted in enhanced remyelination. Collectively, it is suggested that myelination processes can be favorably modulated by decreasing microglia, increasing astrocytes and therefore enhancing and/or preserving oligodendrocytes and, eventually, myelination (Beckmann et al. 2018).

Comparable to $\mathrm{MHC}$ class II, we find that AhR in microglia is dispensable for the cuprizoneinduced demyelination process (Wolf et al. 2018). Instead, it was found that microglial triggering receptor expressed on myeloid cells 2 (TREM2), a surface receptor that binds polyanions and initiates downstream signaling pathways through the adapter DAP12, is required for myelin clearance during cuprizone challenge (Poliani et al. 2015). After extended cuprizone ingestion, Trem $2^{-/-}$mice demonstrated persistent demyelination, impaired removal of damaged myelin, axonal dystrophy and progressive decrease in oligodendrocytes.

Previous studies described a novel mouse model combining the noninflammatory neurodegenerative cuprizone model and the classical autoimmune MS model EAE. In Cup/EAE, mice are pre-intoxicated with cuprizone, leading to severe forebrain inflammation and lesions dominated by infiltrating monocytes, T cells and microglia (Scheld et al. 2016; Ruther et al. 2017; Boretius et al. 2012). This model enables the investigation of focal inflammatory demyelinating lesions, gray matter demyelination, diffuse white matter damage, axonal injury and remyelination (Kipp et al. 2017). Most recently, the group of Markus Kipp found that LAQ treatment mitigates inflammatory demyelination and neurodegeneration in Cup/EAE, which verifies its pronounced protective effects in cuprizoneinduced demyelination (Nedelcu et al. 2020; Brück et al. 2012). In the present study, we observed no impact of AhR deficiency in astrocytes or microglia on cuprizone-induced CNS demyelination, whereas several studies provide evidence that AhR is essential in controlling adaptive CNS inflammation (Rothhammer 2016; Rothhammer et al. 2018). Therefore, the Cup/EAE model represents a promising tool to obtain valuable insights into the role of AhR in astrocytes and microglia during inflammatory lesion development and progression in the forebrain. 


\subsection{Outlook}

The present work demonstrates that astrocytic AhR deletion does not affect cuprizoneinduced CNS demyelination, which also holds true for the outcome of microglial AhR loss, except for oligodendrocyte numbers. LAQ, a presumed AhR agonist, prevented cuprizoneinduced pathology independent of astrocytic and microglial AhR expression. However, some open questions remain. Hence, the following additional experiments would be necessary to fully understand the function of astrocytic and microglial AhR in the cuprizone mouse model and in demyelinating diseases such as MS.

First, based on the literature, one hypothesis on enhanced oligodendrocyte numbers in mice with microglia-specific loss of AhR and in all LAQ-treated animals could be that cuprizonetriggered oligodendrocyte death was decreased. Therefore, it would be interesting to investigate apoptotic oligodendrocytes already after one week of $0.25 \%$ cuprizone treatment, the time point at which oligodendroglial cell death is most pronounced (Hesse et al. 2010). To clarify whether less mature oligodendrocytes die or whether they repopulate faster, sections could be stained for activated caspase-3, a marker for cells undergoing apoptosis, and differences in oligodendrocyte apoptosis could be quantitatively analyzed.

Second, to confirm deletion of AhR in CX3CR1-AhR mice after tamoxifen treatment, expression levels of the AhR target genes CYP1A1 and CYP1B1 could be measured. Primary cells might require additional pre-stimulation with cytokines or LPS because FICZ treatment alone is suggested to be insufficient to activate AhR in microglia (Lee et al. 2015). Such in vitro analyses could clarify whether AhR was efficiently deleted in CX3CR1-positive microglia. Alternatively, it would be intriguing to investigate the functional impact of AhR loss in microglia ex vivo, by using microglia from tamoxifen-induced adult CX3CR1-AhR mice, sorting them after FICZ treatment by flow cytometry and measuring CYP1A1 and CYP1B1 expression levels.

Third, our results regarding astrocytic NF-KB activity stand in part in contradiction to the literature. Therefore, it would be important to analyze whether NF-KB activation in AhRdeficient astrocytes and microglia can be reduced by FICZ treatment. At the same time, glial responses to FICZ stimulation could be compared to LAQ administration by using NF-KB reporter assays. 
Lastly, I demonstrated here that AhR deficiency in astrocytes or microglia has no impact on cuprizone-elicited CNS demyelination. Using the benefits of the cuprizone mouse model, which allows study of de- and remyelination in the CNS by administration or withdrawal of the cuprizone diet (Matsushima 2001), further studies are warranted to examine the role of glial AhR in CNS remyelination after 5-6 weeks of intoxication, with subsequent administration of a normal diet. 


\section{SUMMARY AND CONCLUSIONS}

The aryl hydrocarbon receptor (AhR) is a ligand-activated transcription factor that plays an essential role in the regulation of inflammatory demyelinating diseases of the CNS. It is highly expressed by astrocytes and has been demonstrated to limit inflammation in the CNS. In addition, it was shown that AhR loss in astrocytes and microglia leads to EAE deterioration and failure to recover during the disease (Rothhammer et al. 2016; Rothhammer et al. 2018). Laquinimod (LAQ), an oral drug evaluated for the treatment of MS and presumably an AhR agonist, inhibited toxin-induced demyelination, oligodendroglial apoptosis, microglial infiltration, acute axonal damage and astrogliosis through reduced astrocytic NF-KB activation (Brück et al. 2012). Moreover, it has been shown to protect mice from EAE in an AhRdependent fashion (Kaye et al. 2016).

To better understand the relevance of AhR signaling in glial cells, the present study aimed at investigating the role of astrocytic and microglial AhR in CNS demyelination and determining whether the therapeutic effects of LAQ in cuprizone-induced demyelination are mediated by the AhR. For this, astrocyte- and microglia-specific AhR knockout mice were generated and challenged with the copper chelator cuprizone.

The first part of this thesis examined the impact of AhR deficiency in astrocytes and microglia. To confirm efficient cell-specific AhR deletion in vitro, mRNA expression analyses were performed to examine astrocytic and microglial responses to the AhR ligand FICZ. AhRdeficient primary astrocytes isolated from newborn GFAP- and Aldh1l1-AhR mice displayed strongly decreased expression levels of CYP1A1 after FICZ stimulation relative to control treatment. Thus, we can assume that $A h R$ is successfully deleted in astrocytes from respective animals. To evaluate the impact of astrocytic and microglial AhR deficiency in CNS demyelination, GFAP-, Aldh1l1- and CX3CR1 cell-specific AhR knockout mice were generated and challenged by cuprizone-induced demyelination for 6 weeks. In GFAP-AhR and Aldh1l1AhR knockout mice, both characterized by astrocyte-specific loss of AhR, no effect on the density of mature oligodendrocytes was observed, indicating no interference with cuprizone toxicity. However, microglia-specific loss of AhR resulted in significantly higher numbers of mature oligodendrocytes after 6 weeks of cuprizone intoxication, which might point towards a reduction in oligodendrocyte apoptosis, and slightly ameliorated cuprizone toxicity. Although a 3-week-period of cuprizone administration led to significantly less demyelination 
and microglial activation in CX3CR1-AhR mice, a comparable extent of demyelination, microglial infiltration and axonal damage was observed in all knockout mouse strains and their respective littermate controls after 6 weeks of cuprizone treatment. Thus, the current results suggest that AhR-depletion from astrocytes and microglia has little, or at most, a transient effect on cuprizone-induced CNS demyelination.

The second part of the present study evaluated the role of AhR on astrocytes and microglia as a receptor for LAQ treatment during toxic demyelination. However, administration of LAQ reduced the extent of demyelination, the numbers of acutely damaged axons and activated microglia, as well as rescued the density of mature oligodendrocytes in all three mouse strains independent of genetic background. Thus, LAQ substantially affects cuprizone-induced toxicity and acts - apart from its immunomodulatory properties - as a neuroprotective substance by inhibiting oligodendrocyte death, demyelination, and axonal injury. However, it does not elicit its effect via AhR on astrocytes or microglia. These observations were supported by our findings obtained from a reporter-based in vitro assay. In line with previously published data, showing that LAQ lessens the inflammatory response in astrocytes by attenuation of astrocytic NF-KB activity in vivo and in vitro (Brück et al. 2012), LAQ treatment led to a significantly reduced NF-KB activity in stimulated primary astrocytes, however independent of AhR. In addition, quantitative PCR studies revealed no induction of AhR downstream genes in astrocytes by LAQ administration.

Taken together, the present work demonstrates that astrocytic and microglial AhR is not involved in mediating cuprizone-induced pathology. Remarkably, the LAQ-mediated neuroprotective effects appear to be independent of glial AhR signaling in toxic demyelination. This suggests that the LAQ effect involves other modes of action apart from AhR during cuprizone challenge. Despite a presumably negligible function for AhR in toxic CNS demyelination, the AhR plays a crucial role in LAQ's immunomodulatory efficacy in CNS autoimmunity. In addition, AhR signaling is critically involved in the regulation of the immune response in health and disease. Thus, with the development of relevant advanced medical technology, such as cell type-specific delivery of AhR ligands, the AhR may open a window for future exploration and discovery as a promising therapeutic target, in particular for cancer, inflammatory, and autoimmune diseases. 


\section{REFERENCES}

Albert, M., Antel, J., Brück, W., and Stadelmann, C. 2007. 'Extensive cortical remyelination in patients with chronic multiple sclerosis', Brain Pathol, 17: 129-38.

Bakker, D. A., and Ludwin, S. K. 1987. 'Blood-brain barrier permeability during Cuprizoneinduced demyelination. Implications for the pathogenesis of immune-mediated demyelinating diseases', J Neurol Sci, 78: 125-37.

Beckmann, N., Giorgetti, E., Neuhaus, A., Zurbruegg, S., Accart, N., Smith, P., Perdoux, J., Perrot, L., Nash, M., Desrayaud, S., Wipfli, P., Frieauff, W., and Shimshek, D. R. 2018. 'Brain region-specific enhancement of remyelination and prevention of demyelination by the CSF1R kinase inhibitor BLZ945', Acta Neuropathol Commun, 6: 9.

Ben Haim, L., and Rowitch, D. H. 2017. 'Functional diversity of astrocytes in neural circuit regulation', Nat Rev Neurosci, 18: 31-41.

Benardais, K., Kotsiari, A., Skuljec, J., Koutsoudaki, P. N., Gudi, V., Singh, V., Vulinovic, F., Skripuletz, T., and Stangel, M. 2013. 'Cuprizone [bis(cyclohexylidenehydrazide)] is selectively toxic for mature oligodendrocytes', Neurotox Res, 24: 244-50.

Benveniste, E. N. 1997. 'Cytokines: influence on glial cell gene expression and function', Chem Immunol, 69: 31-75.

Berg, J., Mahmoudjanlou, Y., Duscha, A., Massa, M. G., Thone, J., Esser, C., Gold, R., and Haghikia, A. 2016. 'The immunomodulatory effect of laquinimod in CNS autoimmunity is mediated by the aryl hydrocarbon receptor', J Neuroimmunol, 298: 9-15.

Birnberg, T., Kaye, J., Fowler, K., Weiner, B., Caballero, I., Barash, S., Raymond, E., Ben-Eliezer, I., Fishbein, I., Orbach, A., Laifenfeld, D., Laufer, R., and Grossman, I. 2017. 'Laquinimod targets the aryl hydrocarbon receptor (AhR) pathway in periphery and brains of naïve and EAE mice (P2.365)', Neurology.

Blakemore. 1972. 'Observations on oligodendrocyte degeneration, the resolution of status spongiosus and remyelination in cuprizone intoxication in mice'.

Bo, L., Mork, S., Kong, P. A., Nyland, H., Pardo, C. A., and Trapp, B. D. 1994. 'Detection of MHC class II-antigens on macrophages and microglia, but not on astrocytes and endothelia in active multiple sclerosis lesions', J Neuroimmunol, 51: 135-46.

Boretius, S., Escher, A., Dallenga, T., Wrzos, C., Tammer, R., Brück, W., Nessler, S., Frahm, J., and Stadelmann, C. 2012. 'Assessment of lesion pathology in a new animal model of MS by multiparametric MRI and DTI', Neuroimage, 59: 2678-88.

Brownlee, W. J., Hardy, T. A., Fazekas, F., and Miller, D. H. 2017. 'Diagnosis of multiple sclerosis: progress and challenges', Lancet, 389: 1336-46. 
Brück, W. 2005. 'Clinical implications of neuropathological findings in multiple sclerosis', J Neurol, 252 Suppl 3: iii10-iii14.

Brück, W., Pfortner, R., Pham, T., Zhang, J., Hayardeny, L., Piryatinsky, V., Hanisch, U. K., Regen, T., van Rossum, D., Brakelmann, L., Hagemeier, K., Kuhlmann, T., Stadelmann, C., John, G. R., Kramann, N., and Wegner, C. 2012. 'Reduced astrocytic NF-kappaB activation by laquinimod protects from cuprizone-induced demyelination', Acta Neuropathol, 124: 411-24.

Brück, W., Porada, P., Poser, S., Rieckmann, P., Hanefeld, F., Kretzschmar, H. A., and Lassmann, H. 1995. 'Monocyte/macrophage differentiation in early multiple sclerosis lesions', Ann Neurol, 38: 788-96.

Brück, W., and Stadelmann, C. 2005. 'The spectrum of multiple sclerosis: new lessons from pathology'.

Brück, W., and Vollmer, T. 2013. 'Multiple sclerosis: Oral laquinimod for MS--bringing the brain into focus', Nat Rev Neurol, 9: 664-5.

Brück, W., and Wegner, C. 2011. 'Insight into the mechanism of laquinimod action', J Neurol Sci, 306: 173-9.

Bruttger, J., Karram, K., Wortge, S., Regen, T., Marini, F., Hoppmann, N., Klein, M., Blank, T., Yona, S., Wolf, Y., Mack, M., Pinteaux, E., Muller, W., Zipp, F., Binder, H., Bopp, T., Prinz, M., Jung, S., and Waisman, A. 2015. 'Genetic Cell Ablation Reveals Clusters of Local Self-Renewing Microglia in the Mammalian Central Nervous System', Immunity, 43: $92-$ 106.

Bsibsi, M., Persoon-Deen, C., Verwer, R. W., Meeuwsen, S., Ravid, R., and Van Noort, J. M. 2006. 'Toll-like receptor 3 on adult human astrocytes triggers production of neuroprotective mediators', Glia, 53: 688-95.

Bsibsi, M., Ravid, R., Gveric, D., and van Noort, J. M. 2002. 'Broad expression of Toll-like receptors in the human central nervous system', J Neuropathol Exp Neurol, 61: 101321.

Buniatian, G., Traub, P., Albinus, M., Beckers, G., Buchmann, A., Gebhardt, R., and Osswald, H. 1998. 'The immunoreactivity of glial fibrillary acidic protein in mesangial cells and podocytes of the glomeruli of rat kidney in vivo and in culture', Biol Cell, 90: 53-61.

Bush, T. G., Savidge, T. C., Freeman, T. C., Cox, H. J., Campbell, E. A., Mucke, L., Johnson, M. H., and Sofroniew, M. V. 1998. 'Fulminant jejuno-ileitis following ablation of enteric glia in adult transgenic mice', Cell, 93: 189-201.

Butovsky, O., Jedrychowski, M. P., Moore, C. S., Cialic, R., Lanser, A. J., Gabriely, G., Koeglsperger, T., Dake, B., Wu, P. M., Doykan, C. E., Fanek, Z., Liu, L., Chen, Z., Rothstein, J. D., Ransohoff, R. M., Gygi, S. P., Antel, J. P., and Weiner, H. L. 2014. 'Identification of a unique TGF-beta-dependent molecular and functional signature in microglia', Nat Neurosci, 17: 131-43. 
Buttgereit, A., Lelios, I., Yu, X., Vrohlings, M., Krakoski, N. R., Gautier, E. L., Nishinakamura, R., Becher, B., and Greter, M. 2016. 'Sall1 is a transcriptional regulator defining microglia identity and function', Nat Immunol, 17: 1397-406.

Cahoy, J. D., Emery, B., Kaushal, A., Foo, L. C., Zamanian, J. L., Christopherson, K. S., Xing, Y., Lubischer, J. L., Krieg, P. A., Krupenko, S. A., Thompson, W. J., and Barres, B. A. 2008. 'A transcriptome database for astrocytes, neurons, and oligodendrocytes: a new resource for understanding brain development and function', J Neurosci, 28: 264-78.

Calabrese, M., Oh, M. S., Favaretto, A., Rinaldi, F., Poretto, V., Alessio, S., Lee, B. C., Yu, K. H., Ma, H. I., Perini, P., and Gallo, P. 2012. 'No MRI evidence of cortical lesions in neuromyelitis optica', Neurology, 79: 1671-6.

Caramaschi, F., del Corno, G., Favaretti, C., Giambelluca, S. E., Montesarchio, E., and Fara, G. M. 1981. 'Chloracne following environmental contamination by TCDD in Seveso, Italy', Int J Epidemiol, 10: 135-43.

Carlton, W. W. 1967. 'Studies on the induction of hydrocephalus and spongy degeneration by cuprizone feeding and attempts to antidote the toxicity', Life Sci, 6: 11-9.

Colonna, M. 2014. 'AHR: making the keratinocytes thick skinned', Immunity, 40: 863-4.

Colonna, M., and Butovsky, O. 2017. 'Microglia Function in the Central Nervous System During Health and Neurodegeneration', Annu Rev Immunol, 35: 441-68.

Comi, G. 2012. 'Placebo-Controlled Trial of Oral Laquinimod for Multiple Sclerosis'.

Comi, G., Abramsky, O., Arbizu, T., Boyko, A., Gold, R., Havrdova, E., Komoly, S., Selmaj, K., Sharrack, B., Filippi, M., and Group, L. A. Q. Study. 2010. 'Oral laquinimod in patients with relapsing-remitting multiple sclerosis: 36 -week double-blind active extension of the multi-centre, randomized, double-blind, parallel-group placebo-controlled study', Mult Scler, 16: 1360-6.

Comi, G., Pulizzi, A., Rovaris, M., Abramsky, O., Arbizu, T., Boiko, A., Gold, R., Havrdova, E., Komoly, S., Selmaj, K. W., Sharrack, B., and Filippi, M. 2008. 'Effect of laquinimod on MRI-monitored disease activity in patients with relapsing-remitting multiple sclerosis: a multicentre, randomised, double-blind, placebo-controlled phase IIb study', The Lancet, 371: 2085-92.

Correale, J., and Farez, M. F. 2015. 'The Role of Astrocytes in Multiple Sclerosis Progression', Front Neurol, 6: 180.

Davalos, D., Grutzendler, J., Yang, G., Kim, J. V., Zuo, Y., Jung, S., Littman, D. R., Dustin, M. L., and Gan, W. B. 2005. 'ATP mediates rapid microglial response to local brain injury in vivo', Nat Neurosci, 8: 752-8.

Davidoff, M. S., Middendorff, R., Kofuncu, E., Muller, D., Jezek, D., and Holstein, A. F. 2002. 'Leydig cells of the human testis possess astrocyte and oligodendrocyte marker molecules', Acta Histochem, 104: 39-49. 
De Zeeuw, C. I., and Hoogland, T. M. 2015. 'Reappraisal of Bergmann glial cells as modulators of cerebellar circuit function', Front Cell Neurosci, 9: 246.

Denison, M. S., and Nagy, S. R. 2003. 'Activation of the aryl hydrocarbon receptor by structurally diverse exogenous and endogenous chemicals', Annu Rev Pharmacol Toxicol, 43: 309-34.

Denison, M. S., Pandini, A., Nagy, S. R., Baldwin, E. P., and Bonati, L. 2002. 'Ligand binding and activation of the Ah receptor', Chem Biol Interact, 141: 3-24.

Dever, D. P., Adham, Z. O., Thompson, B., Genestine, M., Cherry, J., Olschowka, J. A., DiCiccoBloom, E., and Opanashuk, L. A. 2016. 'Aryl hydrocarbon receptor deletion in cerebellar granule neuron precursors impairs neurogenesis', Dev Neurobiol, 76: 533-50.

di Domenico, A., Silano, V., Viviano, G., and Zapponi, G. 1980. 'Accidental release of 2,3,7,8tetrachlorodibenzo-p-dioxin (TCDD) at Seveso, Italy. II. TCDD distribution in the soil surface layer', Ecotoxicol Environ Saf, 4: 298-320.

Dobson, R., and Giovannoni, G. 2019. 'Multiple sclerosis - a review', Eur J Neurol, 26: 27-40.

Domingues, H. S., Portugal, C. C., Socodato, R., and Relvas, J. B. 2016. 'Oligodendrocyte, Astrocyte, and Microglia Crosstalk in Myelin Development, Damage, and Repair', Front Cell Dev Biol, 4: 71.

Draheim, T., Liessem, A., Scheld, M., Wilms, F., Weissflog, M., Denecke, B., Kensler, T. W., Zendedel, A., Beyer, C., Kipp, M., Wruck, C. J., Fragoulis, A., and Clarner, T. 2016. 'Activation of the astrocytic Nrf2/ARE system ameliorates the formation of demyelinating lesions in a multiple sclerosis animal model', Glia, 64: 2219-30.

Ellrichmann, G., Blusch, A., Fatoba, O., Brunner, J., Reick, C., Hayardeny, L., Hayden, M., Sehr, D., Winklhofer, K. F., Saft, C., and Gold, R. 2017. 'Laquinimod treatment in the R6/2 mouse model', Sci Rep, 7: 4947.

Elmore, M. R., Najafi, A. R., Koike, M. A., Dagher, N. N., Spangenberg, E. E., Rice, R. A., Kitazawa, M., Matusow, B., Nguyen, H., West, B. L., and Green, K. N. 2014. 'Colony-stimulating factor 1 receptor signaling is necessary for microglia viability, unmasking a microglia progenitor cell in the adult brain', Neuron, 82: 380-97.

EMA. 2014. 'Refusal of the marketing authorisation for Nerventra (laquinimod).', Accessed 15 July 2020. http://www.ema.europa.eu/docs/en_GB/document_library/Summary_of_opinion__Initial_authorisation/human/002546/WC500160120.pdf.

Esser, C. 2009. 'The immune phenotype of AhR null mouse mutants: not a simple mirror of xenobiotic receptor over-activation', Biochem Pharmacol, 77: 597-607.

Esser, C., and Rannug, A. 2015. 'The aryl hydrocarbon receptor in barrier organ physiology, immunology, and toxicology', Pharmacol Rev, 67: 259-79.

Esser, C., Rannug, A., and Stockinger, B. 2009. 'The aryl hydrocarbon receptor in immunity', Trends Immunol, 30: 447-54. 
Ferguson, B., Matyszak, M. K., Esiri, M. M., and Perry, V. H. 1997. 'Axonal damage in acute multiple sclerosis lesions', Brain, 120 ( Pt 3): 393-9.

Fernandez-Salguero, P. M., Ward, J. M., Sundberg, J. P., and Gonzalez, F. J. 1997. 'Lesions of aryl-hydrocarbon receptor-deficient mice', Vet Pathol, 34: 605-14.

Fernandez-Salguero, P., Pineau, T., Hilbert, D. M., McPhail, T., Lee, S. S., Kimura, S., Nebert, D. W., Rudikoff, S., Ward, J. M., and Gonzalez, F. J. 1995. 'Immune system impairment and hepatic fibrosis in mice lacking the dioxin-binding Ah receptor', Science, 268: 722-6.

Filippi, M., Bar-Or, A., Piehl, F., Preziosa, P., Solari, A., Vukusic, S., and Rocca, M. A. 2018. 'Multiple sclerosis', Nat Rev Dis Primers, 4: 43.

Frischer, J. M., Weigand, S. D., Guo, Y., Kale, N., Parisi, J. E., Pirko, I., Mandrekar, J., Bramow, S., Metz, I., Brück, W., Lassmann, H., and Lucchinetti, C. F. 2015. 'Clinical and pathological insights into the dynamic nature of the white matter multiple sclerosis plaque', Ann Neurol, 78: 710-21.

Fulmer, C. G., VonDran, M. W., Stillman, A. A., Huang, Y., Hempstead, B. L., and Dreyfus, C. F. 2014. 'Astrocyte-derived BDNF supports myelin protein synthesis after cuprizoneinduced demyelination', J Neurosci, 34: 8186-96.

Gandhi, R., Laroni, A., and Weiner, H. L. 2010. 'Role of the innate immune system in the pathogenesis of multiple sclerosis', J Neuroimmunol, 221: 7-14.

Garcia, A. D., Doan, N. B., Imura, T., Bush, T. G., and Sofroniew, M. V. 2004. 'GFAP-expressing progenitors are the principal source of constitutive neurogenesis in adult mouse forebrain', Nat Neurosci, 7: 1233-41.

Garcia-Miralles, M., Yusof, Nabm, Tan, J. Y., Radulescu, C. I., Sidik, H., Tan, L. J., Belinson, H., Zach, N., Hayden, M. R., and Pouladi, M. A. 2019. 'Laquinimod Treatment Improves Myelination Deficits at the Transcriptional and Ultrastructural Levels in the YAC128 Mouse Model of Huntington Disease', Mol Neurobiol, 56: 4464-78.

Giovannoni, G., Barkhof, F., Hartung, H., Cree, B., Krieger, S., Montalban, X., Sormani, M., Uccelli, A., Uitdehaag, B., Vollmer, T., Reshef, A., Li, T., Feldman, P., Tansy, A., and Steinerman, J. 2018. 'ARPEGGIO: a placebo-controlled trial of oral laquinimod in primary progressive multiple sclerosis (S8.003)', Neurology.

Goldmann, T., and Prinz, M. 2013. 'Role of microglia in CNS autoimmunity', Clin Dev Immunol, 2013: 208093.

Greter, M., Heppner, F. L., Lemos, M. P., Odermatt, B. M., Goebels, N., Laufer, T., Noelle, R. J., and Becher, B. 2005. 'Dendritic cells permit immune invasion of the CNS in an animal model of multiple sclerosis', Nat Med, 11: 328-34.

Gudi, V., Gingele, S., Skripuletz, T., and Stangel, M. 2014. 'Glial response during cuprizoneinduced de- and remyelination in the CNS: lessons learned', Front Cell Neurosci, 8: 73. 
Gudi, V., Moharregh-Khiabani, D., Skripuletz, T., Koutsoudaki, P. N., Kotsiari, A., Skuljec, J., Trebst, C., and Stangel, M. 2009. 'Regional differences between grey and white matter in cuprizone induced demyelination', Brain Res, 1283: 127-38.

Hagemeyer, N., Hanft, K. M., Akriditou, M. A., Unger, N., Park, E. S., Stanley, E. R., Staszewski, O., Dimou, L., and Prinz, M. 2017. 'Microglia contribute to normal myelinogenesis and to oligodendrocyte progenitor maintenance during adulthood', Acta Neuropathol, 134: 441-58.

Hemm, R. D., Carlton, W. W., and Welser, J. R. 1971. 'Ultrastructural changes of cuprizone encephalopathy in mice', Toxicol Appl Pharmacol, 18: 869-82.

Hesse, A., Wagner, M., Held, J., Brück, W., Salinas-Riester, G., Hao, Z., Waisman, A., and Kuhlmann, T. 2010. 'In toxic demyelination oligodendroglial cell death occurs early and is FAS independent', Neurobiol Dis, 37: 362-9.

Hiremath, M. M., Saito, Y., Knapp, G. W., Ting, J. P., Suzuki, K., and Matsushima, G. K. 1998. 'Microglial/macrophage accumulation during cuprizone-induced demyelination in C57BL/6 mice', J Neuroimmunol, 92: 38-49.

Iba, M. M., Scholl, H., Fung, J., Thomas, P. E., and Alam, J. 1998. 'Induction of pulmonary CYP1A1 by nicotine', Xenobiotica, 28: 827-43.

Irvine, K. A., and Blakemore, W. F. 2006. 'Age increases axon loss associated with primary demyelination in cuprizone-induced demyelination in C57BL/6 mice', J Neuroimmunol, 175: 69-76.

Jack, C. S., Arbour, N., Manusow, J., Montgrain, V., Blain, M., McCrea, E., Shapiro, A., and Antel, J. P. 2005. 'TLR signaling tailors innate immune responses in human microglia and astrocytes', J Immunol, 175: 4320-30.

Jessen, K. R., Morgan, L., Stewart, H. J., and Mirsky, R. 1990. 'Three markers of adult nonmyelin-forming Schwann cells, 217c(Ran-1), A5E3 and GFAP: development and regulation by neuron-Schwann cell interactions', Development, 109: 91-103.

Jonsson, S., Andersson, G., Fex, T., Fristedt, T., Hedlund, G., Jansson, K., Abramo, L., Fritzson, I., Pekarski, O., Runstrom, A., Sandin, H., Thuvesson, I., and Bjork, A. 2004. 'Synthesis and biological evaluation of new 1,2-dihydro-4-hydroxy-2-oxo-3quinolinecarboxamides for treatment of autoimmune disorders: structure-activity relationship', J Med Chem, 47: 2075-88.

Jux, B., Kadow, S., and Esser, C. 2009. 'Langerhans cell maturation and contact hypersensitivity are impaired in aryl hydrocarbon receptor-null mice', J Immunol, 182: 6709-17.

Kainu, T., Gustafsson, J. A., and Pelto-Huikko, M. 1995. 'The dioxin receptor and its nuclear translocator (Arnt) in the rat brain', Neuroreport, 6: 2557-60.

Kang, Z., Liu, L., Spangler, R., Spear, C., Wang, C., Gulen, M. F., Veenstra, M., Ouyang, W., Ransohoff, R. M., and Li, X. 2012. 'IL-17-induced Act1-mediated signaling is critical for cuprizone-induced demyelination', J Neurosci, 32: 8284-92. 
Karni, A., Abraham, M., Monsonego, A., Cai, G., Freeman, G. J., Hafler, D., Khoury, S. J., and Weiner, H. L. 2006. 'Innate immunity in multiple sclerosis: myeloid dendritic cells in secondary progressive multiple sclerosis are activated and drive a proinflammatory immune response', J Immunol, 177: 4196-202.

Katsumoto, A., Miranda, A. S., Butovsky, O., Teixeira, A. L., Ransohoff, R. M., and Lamb, B. T. 2018. 'Laquinimod attenuates inflammation by modulating macrophage functions in traumatic brain injury mouse model', J Neuroinflammation, 15: 26.

Kaye, J., Piryatinsky, V., Birnberg, T., Hingaly, T., Raymond, E., Kashi, R., Amit-Romach, E., Caballero, I. S., Towfic, F., Ator, M. A., Rubinstein, E., Laifenfeld, D., Orbach, A., Shinar, D., Marantz, Y., Grossman, I., Knappertz, V., Hayden, M. R., and Laufer, R. 2016. 'Laquinimod arrests experimental autoimmune encephalomyelitis by activating the aryl hydrocarbon receptor', Proc Natl Acad Sci U S A, 113: E6145-E52.

Kettenmann, H., Hanisch, U. K., Noda, M., and Verkhratsky, A. 2011. 'Physiology of microglia', Physiol Rev, 91: 461-553.

Kim, R. Y., Hoffman, A. S., Itoh, N., Ao, Y., Spence, R., Sofroniew, M. V., and Voskuhl, R. R. 2014. 'Astrocyte CCL2 sustains immune cell infiltration in chronic experimental autoimmune encephalomyelitis', J Neuroimmunol, 274: 53-61.

Kimura, A., Naka, T., Nakahama, T., Chinen, I., Masuda, K., Nohara, K., Fujii-Kuriyama, Y., and Kishimoto, T. 2009. 'Aryl hydrocarbon receptor in combination with Stat1 regulates LPS-induced inflammatory responses', J Exp Med, 206: 2027-35.

Kipp, M., Clarner, T., Dang, J., Copray, S., and Beyer, C. 2009. 'The cuprizone animal model: new insights into an old story', Acta Neuropathol, 118: 723-36.

Kipp, M., Nyamoya, S., Hochstrasser, T., and Amor, S. 2017. 'Multiple sclerosis animal models: a clinical and histopathological perspective', Brain Pathol, 27: 123-37.

Komoly, S., Jeyasingham, M. D., Pratt, O. E., and Lantos, P. L. 1987. 'Decrease in oligodendrocyte carbonic anhydrase activity preceding myelin degeneration in cuprizone induced demyelination', J Neurol Sci, 79: 141-8.

Kramann, N., Menken, L., Hayardeny, L., Hanisch, U. K., and Brück, W. 2016. 'Laquinimod prevents cuprizone-induced demyelination independent of Toll-like receptor signaling', Neurol Neuroimmunol Neuroinflamm, 3: e233.

Kramann, N., Menken, L., Pfortner, R., Schmid, S. N., Stadelmann, C., Wegner, C., and Brück, W. 2019. 'Glial fibrillary acidic protein expression alters astrocytic chemokine release and protects mice from cuprizone-induced demyelination', Glia, 67: 1308-19.

Kriszta, G., Nemes, B., Sandor, Z., Acs, P., Komoly, S., Berente, Z., Bolcskei, K., and Pinter, E. 2019. 'Investigation of Cuprizone-Induced Demyelination in mGFAP-Driven Conditional Transient Receptor Potential Ankyrin 1 (TRPA1) Receptor Knockout Mice', Cells, 9. 
Krupenko, S. A., and Oleinik, N. V. 2002. '10-formyltetrahydrofolate dehydrogenase, one of the major folate enzymes, is down-regulated in tumor tissues and possesses suppressor effects on cancer cells', Cell Growth Differ, 13: 227-36.

Kuhlmann, T., Ludwin, S., Prat, A., Antel, J., Brück, W., and Lassmann, H. 2017. 'An updated histological classification system for multiple sclerosis lesions', Acta Neuropathol, 133: 13-24.

Kutzelnigg, A., and Lassmann, H. 2014. 'Pathology of multiple sclerosis and related inflammatory demyelinating diseases', Handb Clin Neurol, 122: 15-58.

Kutzelnigg, A., Lucchinetti, C. F., Stadelmann, C., Brück, W., Rauschka, H., Bergmann, M., Schmidbauer, M., Parisi, J. E., and Lassmann, H. 2005. 'Cortical demyelination and diffuse white matter injury in multiple sclerosis', Brain, 128: 2705-12.

Laflamme, N., Cisbani, G., Prefontaine, P., Srour, Y., Bernier, J., St-Pierre, M. K., Tremblay, M. E., and Rivest, S. 2018. 'mCSF-Induced Microglial Activation Prevents Myelin Loss and Promotes Its Repair in a Mouse Model of Multiple Sclerosis', Front Cell Neurosci, 12: 178.

Lahvis, G. P., and Bradfield, C. A. 1998. 'Ahr null alleles: distinctive or different?', Biochem Pharmacol, 56: 781-7.

Lampron, A., Larochelle, A., Laflamme, N., Prefontaine, P., Plante, M. M., Sanchez, M. G., Yong, V. W., Stys, P. K., Tremblay, M. E., and Rivest, S. 2015. 'Inefficient clearance of myelin debris by microglia impairs remyelinating processes', J Exp Med, 212: 481-95.

Larochelle, C., Alvarez, J. I., and Prat, A. 2011. 'How do immune cells overcome the blood-brain barrier in multiple sclerosis?', FEBS Lett, 585: 3770-80.

Lassmann, H., Brück, W., and Lucchinetti, C. F. 2007. 'The immunopathology of multiple sclerosis: an overview', Brain Pathol, 17: 210-8.

Lassmann, H., van Horssen, J., and Mahad, D. 2012. 'Progressive multiple sclerosis: pathology and pathogenesis', Nat Rev Neurol, 8: 647-56.

Lee, D. H., Geyer, E., Flach, A. C., Jung, K., Gold, R., Flugel, A., Linker, R. A., and Luhder, F. 2012. 'Central nervous system rather than immune cell-derived BDNF mediates axonal protective effects early in autoimmune demyelination', Acta Neuropathol, 123: 24758.

Lee, Y. H., Lin, C. H., Hsu, P. C., Sun, Y. Y., Huang, Y. J., Zhuo, J. H., Wang, C. Y., Gan, Y. L., Hung, C. C., Kuan, C. Y., and Shie, F. S. 2015. 'Aryl hydrocarbon receptor mediates both proinflammatory and anti-inflammatory effects in lipopolysaccharide-activated microglia', Glia, 63: 1138-54.

Li, T., and Zhang, S. 2016. 'Microgliosis in the Injured Brain: Infiltrating Cells and Reactive Microglia Both Play a Role', Neuroscientist, 22: 165-70. 
Li, W., Donat, S., Dohr, O., Unfried, K., and Abel, J. 1994. 'Ah receptor in different tissues of C57BL/6J and DBA/2J mice: use of competitive polymerase chain reaction to measure Ah-receptor mRNA expression', Arch Biochem Biophys, 315: 279-84.

Liddelow, S. A., Guttenplan, K. A., Clarke, L. E., Bennett, F. C., Bohlen, C. J., Schirmer, L., Bennett, M. L., Munch, A. E., Chung, W. S., Peterson, T. C., Wilton, D. K., Frouin, A., Napier, B. A., Panicker, N., Kumar, M., Buckwalter, M. S., Rowitch, D. H., Dawson, V. L., Dawson, T. M., Stevens, B., and Barres, B. A. 2017. 'Neurotoxic reactive astrocytes are induced by activated microglia', Nature, 541: 481-87.

Lim, M. C., Maubach, G., and Zhuo, L. 2008. 'Glial fibrillary acidic protein splice variants in hepatic stellate cells--expression and regulation', Mol Cells, 25: 376-84.

Littman, DR. 2013. 'An inducible cre recombinase driven by Cx3cr1', MGI Direct Data Submission.

Lloyd, A. F., and Miron, V. E. 2019. 'The pro-remyelination properties of microglia in the central nervous system', Nat Rev Neurol, 15: 447-58.

Lourenco, E. V., Wong, M., Hahn, B. H., Palma-Diaz, M. F., and Skaggs, B. J. 2014. 'Laquinimod delays and suppresses nephritis in lupus-prone mice and affects both myeloid and lymphoid immune cells', Arthritis Rheumatol, 66: 674-85.

Lublin, F. D., and Reingold, S. C. 1996. 'Defining the clinical course of multiple sclerosis: results of an international survey. National Multiple Sclerosis Society (USA) Advisory Committee on Clinical Trials of New Agents in Multiple Sclerosis', Neurology, 46: 90711.

Lublin, F. D., Reingold, S. C., Cohen, J. A., Cutter, G. R., Sorensen, P. S., Thompson, A. J., Wolinsky, J. S., Balcer, L. J., Banwell, B., Barkhof, F., Bebo, B., Jr., Calabresi, P. A., Clanet, M., Comi, G., Fox, R. J., Freedman, M. S., Goodman, A. D., Inglese, M., Kappos, L., Kieseier, B. C., Lincoln, J. A., Lubetzki, C., Miller, A. E., Montalban, X., O'Connor, P. W., Petkau, J., Pozzilli, C., Rudick, R. A., Sormani, M. P., Stuve, O., Waubant, E., and Polman, C. H. 2014. 'Defining the clinical course of multiple sclerosis: the 2013 revisions', Neurology, 83: 278-86.

Lucchinetti, C. F. 2000. 'Heterogeneity of Multiple Sclerosis Lesions: Implications for the Pathogenesis of Demyelination'.

MacGregor, J. I., and Jordan, V. C. 1998. 'Basic guide to the mechanisms of antiestrogen action', Pharmacol Rev, 50: 151-96.

Machado-Santos, J., Saji, E., Troscher, A. R., Paunovic, M., Liblau, R., Gabriely, G., Bien, C. G., Bauer, J., and Lassmann, H. 2018. 'The compartmentalized inflammatory response in the multiple sclerosis brain is composed of tissue-resident CD8+ T lymphocytes and $B$ cells', Brain, 141: 2066-82.

Manrique-Hoyos, N., Jurgens, T., Gronborg, M., Kreutzfeldt, M., Schedensack, M., Kuhlmann, T., Schrick, C., Brück, W., Urlaub, H., Simons, M., and Merkler, D. 2012. 'Late motor 
decline after accomplished remyelination: impact for progressive multiple sclerosis', Ann Neurol, 71: 227-44.

Marcus, R. S., Holsapple, M. P., and Kaminski, N. E. 1998. 'Lipopolysaccharide activation of murine splenocytes and splenic B cells increased the expression of aryl hydrocarbon receptor and aryl hydrocarbon receptor nuclear translocator', J Pharmacol Exp Ther, 287: 1113-8.

Mason, J. L., Jones, J. J., Taniike, M., Morell, P., Suzuki, K., and Matsushima, G. K. 2000. 'Mature oligodendrocyte apoptosis precedes IGF-1 production and oligodendrocyte progenitor accumulation and differentiation during demyelination/remyelination', J Neurosci Res, 61: 251-62.

Matsushima. 2001. 'The Neurotoxicant, Cuprizone, as a Model to Study Demyelination and Remyelination in the Central Nervous System'.

Mayo, L., Quintana, F. J., and Weiner, H. L. 2012. 'The innate immune system in demyelinating disease', Immunol Rev, 248: 170-87.

Mc Guire, C., Prinz, M., Beyaert, R., and van Loo, G. 2013. 'Nuclear factor kappa B (NF-kappaB) in multiple sclerosis pathology', Trends Mol Med, 19: 604-13.

McDonald, W. I., Compston, A., Edan, G., Goodkin, D., Hartung, H. P., Lublin, F. D., McFarland, H. F., Paty, D. W., Polman, C. H., Reingold, S. C., Sandberg-Wollheim, M., Sibley, W., Thompson, A., van den Noort, S., Weinshenker, B. Y., and Wolinsky, J. S. 2001. 'Recommended diagnostic criteria for multiple sclerosis: guidelines from the International Panel on the diagnosis of multiple sclerosis', Ann Neurol, 50: 121-7.

Miller, D., Barkhof, F., Montalban, X., Thompson, A., and Filippi, M. 2005. 'Clinically isolated syndromes suggestive of multiple sclerosis, part I: natural history, pathogenesis, diagnosis, and prognosis', Lancet Neurol, 4: 281-8.

Mimura, J., Yamashita, K., Nakamura, K., Morita, M., Takagi, T. N., Nakao, K., Ema, M., Sogawa, K., Yasuda, M., Katsuki, M., and Fujii-Kuriyama, Y. 1997. 'Loss of teratogenic response to 2,3,7,8-tetrachlorodibenzo-p-dioxin (TCDD) in mice lacking the Ah (dioxin) receptor', Genes Cells, 2: 645-54.

Mishra, M. K., Wang, J., Keough, M. B., Fan, Y., Silva, C., Sloka, S., Hayardeny, L., Brück, W., and Yong, V. W. 2014. 'Laquinimod reduces neuroaxonal injury through inhibiting microglial activation', Ann Clin Transl Neurol, 1: 409-22.

Molofsky, A. V., Glasgow, S. M., Chaboub, L. S., Tsai, H. H., Murnen, A. T., Kelley, K. W., Fancy, S. P., Yuen, T. J., Madireddy, L., Baranzini, S., Deneen, B., Rowitch, D. H., and Oldham, M. C. 2013. 'Expression profiling of Aldh1l1-precursors in the developing spinal cord reveals glial lineage-specific genes and direct Sox9-Nfe2I1 interactions', Glia, 61: 151832.

Monteleone, I., Rizzo, A., Sarra, M., Sica, G., Sileri, P., Biancone, L., MacDonald, T. T., Pallone, F., and Monteleone, G. 2011. 'Aryl hydrocarbon receptor-induced signals up-regulate 
IL-22 production and inhibit inflammation in the gastrointestinal tract', Gastroenterology, 141: 237-48, 48 e1.

Moore, S., Khalaj, A. J., Yoon, J., Patel, R., Hannsun, G., Yoo, T., Sasidhar, M., Martinez-Torres, L., Hayardeny, L., and Tiwari-Woodruff, S. K. 2013. 'Therapeutic laquinimod treatment decreases inflammation, initiates axon remyelination, and improves motor deficit in a mouse model of multiple sclerosis', Brain Behav, 3: 664-82.

Nair, A., Frederick, T. J., and Miller, S. D. 2008. 'Astrocytes in multiple sclerosis: a product of their environment', Cell Mol Life Sci, 65: 2702-20.

Nedelcu, J., Reinbach, C., Riedler, P., Brendel, M., Rominger, A., Kaye, J., Behrangi, N., Jiangshan, Z., Schmitz, C., and Kipp, M. 2020. 'Laquinimod ameliorates secondary brain inflammation', Neurobiol Dis, 134: 104675.

Neymeyer, V., Tephly, T. R., and Miller, M. W. 1997. 'Folate and 10-formyltetrahydrofolate dehydrogenase (FDH) expression in the central nervous system of the mature rat', Brain Res, 766: 195-204.

Nguyen, N. T., Nakahama, T., and Kishimoto, T. 2013. 'Aryl hydrocarbon receptor and experimental autoimmune arthritis', Semin Immunopathol, 35: 637-44.

Nimmerjahn, A., Kirchhoff, F., and Helmchen, F. 2005. 'Resting microglial cells are highly dynamic surveillants of brain parenchyma in vivo', Science, 308: 1314-8.

Noseworthy, J. H., Wolinsky, J. S., Lublin, F. D., Whitaker, J. N., Linde, A., Gjorstrup, P., and Sullivan, H. C. 2000. 'Linomide in relapsing and secondary progressive MS: part I: trial design and clinical results. North American Linomide Investigators', Neurology, 54: 1726-33.

Nyamoya, S., Steinle, J., Chrzanowski, U., Kaye, J., Schmitz, C., Beyer, C., and Kipp, M. 2019. 'Laquinimod Supports Remyelination in Non-Supportive Environments', Cells, 8.

Obermeier, B., Daneman, R., and Ransohoff, R. M. 2013. 'Development, maintenance and disruption of the blood-brain barrier', Nat Med, 19: 1584-96.

Ohtake, F., Takeyama, K., Matsumoto, T., Kitagawa, H., Yamamoto, Y., Nohara, K., Tohyama, C., Krust, A., Mimura, J., Chambon, P., Yanagisawa, J., Fujii-Kuriyama, Y., and Kato, S. 2003. 'Modulation of oestrogen receptor signalling by association with the activated dioxin receptor', Nature, 423: 545-50.

Ortiz, G. G., Pacheco-Moises, F. P., Macias-Islas, M. A., Flores-Alvarado, L. J., Mireles-Ramirez, M. A., Gonzalez-Renovato, E. D., Hernandez-Navarro, V. E., Sanchez-Lopez, A. L., and Alatorre-Jimenez, M. A. 2014. 'Role of the blood-brain barrier in multiple sclerosis', Arch Med Res, 45: 687-97.

Ott, M., Avendano-Guzman, E., Ullrich, E., Dreyer, C., Strauss, J., Harden, M., Schon, M., Schon, M. P., Bernhardt, G., Stadelmann, C., Wegner, C., Brück, W., and Nessler, S. 2019. 'Laquinimod, a prototypic quinoline-3-carboxamide and aryl hydrocarbon receptor 
agonist, utilizes a CD155-mediated natural killer/dendritic cell interaction to suppress CNS autoimmunity', J Neuroinflammation, 16: 49.

Parkhurst, C. N., Yang, G., Ninan, I., Savas, J. N., Yates, J. R., 3rd, Lafaille, J. J., Hempstead, B. L., Littman, D. R., and Gan, W. B. 2013. 'Microglia promote learning-dependent synapse formation through brain-derived neurotrophic factor', Cell, 155: 1596-609.

Pasquini, L. A., Calatayud, C. A., Bertone Una, A. L., Millet, V., Pasquini, J. M., and Soto, E. F. 2007. 'The neurotoxic effect of cuprizone on oligodendrocytes depends on the presence of pro-inflammatory cytokines secreted by microglia', Neurochem Res, 32: 279-92.

Pekny, M., and Nilsson, M. 2005. 'Astrocyte activation and reactive gliosis', Glia, 50: 427-34.

Pekny, M., and Pekna, M. 2004. 'Astrocyte intermediate filaments in CNS pathologies and regeneration', J Pathol, 204: 428-37.

Peterson, J. W., Bo, L., Mork, S., Chang, A., and Trapp, B. D. 2001. 'Transected neurites, apoptotic neurons, and reduced inflammation in cortical multiple sclerosis lesions', Ann Neurol, 50: 389-400.

Platten, M., Ho, P. P., Youssef, S., Fontoura, P., Garren, H., Hur, E. M., Gupta, R., Lee, L. Y., Kidd, B. A., Robinson, W. H., Sobel, R. A., Selley, M. L., and Steinman, L. 2005. 'Treatment of autoimmune neuroinflammation with a synthetic tryptophan metabolite', Science, 310: 850-5.

Poliani, P. L., Wang, Y., Fontana, E., Robinette, M. L., Yamanishi, Y., Gilfillan, S., and Colonna, M. 2015. 'TREM2 sustains microglial expansion during aging and response to demyelination', J Clin Invest, 125: 2161-70.

Polman, C., Barkhof, F., Sandberg-Wollheim, M., Linde, A., Nordle, O., Nederman, T., and Laquinimod in Relapsing, M. S. Study Group. 2005. 'Treatment with laquinimod reduces development of active MRI lesions in relapsing MS', Neurology, 64: 987-91.

Polman, C. H., Reingold, S. C., Banwell, B., Clanet, M., Cohen, J. A., Filippi, M., Fujihara, K., Havrdova, E., Hutchinson, M., Kappos, L., Lublin, F. D., Montalban, X., O'Connor, P., Sandberg-Wollheim, M., Thompson, A. J., Waubant, E., Weinshenker, B., and Wolinsky, J. S. 2011. 'Diagnostic criteria for multiple sclerosis: 2010 revisions to the McDonald criteria', Ann Neurol, 69: 292-302.

Ponath, G., Park, C., and Pitt, D. 2018. 'The Role of Astrocytes in Multiple Sclerosis', Front Immunol, 9: 217.

Pons, V., Laflamme, N., Prefontaine, P., and Rivest, S. 2020. 'Role of Macrophage ColonyStimulating Factor Receptor on the Proliferation and Survival of Microglia Following Systemic Nerve and Cuprizone-Induced Injuries', Front Immunol, 11: 47.

Popescu, B. F., and Lucchinetti, C. F. 2012. 'Pathology of demyelinating diseases', Annu Rev Pathol, 7: 185-217. 
Praet, J., Guglielmetti, C., Berneman, Z., Van der Linden, A., and Ponsaerts, P. 2014. 'Cellular and molecular neuropathology of the cuprizone mouse model: clinical relevance for multiple sclerosis', Neurosci Biobehav Rev, 47: 485-505.

Prineas, JW. 1985. "The neuropathology of multiple sclerosis." In Handbook of clinical neurology, edited by Koetsier JC, pp. 337-95. Elsevier, New York.

Prinz, M., and Priller, J. 2014. 'Microglia and brain macrophages in the molecular age: from origin to neuropsychiatric disease', Nat Rev Neurosci, 15: 300-12.

Qiu, J., Heller, J. J., Guo, X., Chen, Z. M., Fish, K., Fu, Y. X., and Zhou, L. 2012. 'The aryl hydrocarbon receptor regulates gut immunity through modulation of innate lymphoid cells', Immunity, 36: 92-104.

Quintana, F. J., and Sherr, D. H. 2013. 'Aryl hydrocarbon receptor control of adaptive immunity', Pharmacol Rev, 65: 1148-61.

Raasch, J., Zeller, N., van Loo, G., Merkler, D., Mildner, A., Erny, D., Knobeloch, K. P., Bethea, J. R., Waisman, A., Knust, M., Del Turco, D., Deller, T., Blank, T., Priller, J., Brück, W., Pasparakis, M., and Prinz, M. 2011. 'IkappaB kinase 2 determines oligodendrocyte loss by non-cell-autonomous activation of NF-kappaB in the central nervous system', Brain, 134: 1184-98.

Ramagopalan, S. V., Dobson, R., Meier, U. C., and Giovannoni, G. 2010. 'Multiple sclerosis: risk factors, prodromes, and potential causal pathways', Lancet Neurol, 9: 727-39.

Ramón Y Cajal, S. 1909. Histologie du Système Nerveux de L'Homme \& des Vertébrés (Paris: Maloine).

Rannug, A., Rannug, U., Rosenkranz, H. S., Winqvist, L., Westerholm, R., Agurell, E., and Grafstrom, A. K. 1987. 'Certain photooxidized derivatives of tryptophan bind with very high affinity to the Ah receptor and are likely to be endogenous signal substances', J Biol Chem, 262: 15422-7.

Regoli, M., Orazioli, D., Gerli, R., and Bertelli, E. 2000. 'Glial fibrillary acidic protein (GFAP)-like immunoreactivity in rat endocrine pancreas', J Histochem Cytochem, 48: 259-66.

Reich, D. S., Lucchinetti, C. F., and Calabresi, P. A. 2018. 'Multiple Sclerosis', N Engl J Med, 378 : 169-80.

Reilmann, R., Forrest Gordon, M., Anderson, K. E., Andrew Feigin, A., Tabrizi, S. J., Leavitt, B. R., Stout, J. C., Piccini, P., Borowsky, B., Rynkowski, G., Volkinstein, R., Savola, J., and Hayden, M. R. 2019. 'The Efficacy and Safety Results of Laquinimod as a Treatment for Huntington Disease (LEGATO-HD) (S16.007)', Neurology.

Remington, L. T., Babcock, A. A., Zehntner, S. P., and Owens, T. 2007. 'Microglial recruitment, activation, and proliferation in response to primary demyelination', Am J Pathol, 170: 1713-24. 
Rothhammer, V. 2016. 'Type I interferons and microbial metabolites of tryptophan modulate astrocyte activity and central nervous system inflammation via the aryl hydrocarbon receptor - Supplement'.

Rothhammer, V., Borucki, D. M., Tjon, E. C., Takenaka, M. C., Chao, C. C., Ardura-Fabregat, A., de Lima, K. A., Gutierrez-Vazquez, C., Hewson, P., Staszewski, O., Blain, M., Healy, L., Neziraj, T., Borio, M., Wheeler, M., Dragin, L. L., Laplaud, D. A., Antel, J., Alvarez, J. I., Prinz, M., and Quintana, F. J. 2018. 'Microglial control of astrocytes in response to microbial metabolites', Nature, 557: 724-28.

Rothhammer, V., Mascanfroni, I. D., Bunse, L., Takenaka, M. C., Kenison, J. E., Mayo, L., Chao, C. C., Patel, B., Yan, R., Blain, M., Alvarez, J. I., Kebir, H., Anandasabapathy, N., Izquierdo, G., Jung, S., Obholzer, N., Pochet, N., Clish, C. B., Prinz, M., Prat, A., Antel, J., and Quintana, F. J. 2016. 'Type I interferons and microbial metabolites of tryptophan modulate astrocyte activity and central nervous system inflammation via the aryl hydrocarbon receptor', Nat Med, 22: 586-97.

Rothhammer, V., and Quintana, F. J. 2019. 'The aryl hydrocarbon receptor: an environmental sensor integrating immune responses in health and disease', Nat Rev Immunol, 19: 184-97.

Ruffini, F., Rossi, S., Bergamaschi, A., Brambilla, E., Finardi, A., Motta, C., Studer, V., Barbieri, F., De Chiara, V., Hayardeny, L., Comi, G., Centonze, D., and Martino, G. 2013. 'Laquinimod prevents inflammation-induced synaptic alterations occurring in experimental autoimmune encephalomyelitis', Mult Scler, 19: 1084-94.

Ruther, B. J., Scheld, M., Dreymueller, D., Clarner, T., Kress, E., Brandenburg, L. O., Swartenbroekx, T., Hoornaert, C., Ponsaerts, P., Fallier-Becker, P., Beyer, C., Rohr, S. O., Schmitz, C., Chrzanowski, U., Hochstrasser, T., Nyamoya, S., and Kipp, M. 2017. 'Combination of cuprizone and experimental autoimmune encephalomyelitis to study inflammatory brain lesion formation and progression', Glia, 65: 1900-13.

Safe, S., and McDougal, A. 2002. 'Mechanism of action and development of selective aryl hydrocarbon receptor modulators for treatment of hormone-dependent cancers (Review)', Int J Oncol, 20: 1123-8.

Scheld, M., Ruther, B. J., Grosse-Veldmann, R., Ohl, K., Tenbrock, K., Dreymuller, D., FallierBecker, P., Zendedel, A., Beyer, C., Clarner, T., and Kipp, M. 2016. 'Neurodegeneration Triggers Peripheral Immune Cell Recruitment into the Forebrain', J Neurosci, 36: 14105.

Schmidt, J. V., and Bradfield, C. A. 1996. 'Ah receptor signaling pathways', Annu Rev Cell Dev Biol, 12: 55-89.

Schmidt, J. V., Su, G. H., Reddy, J. K., Simon, M. C., and Bradfield, C. A. 1996. 'Characterization of a murine Ahr null allele: involvement of the Ah receptor in hepatic growth and development', Proc Natl Acad Sci U S A, 93: 6731-6. 
Schultz, V., van der Meer, F., Wrzos, C., Scheidt, U., Bahn, E., Stadelmann, C., Brück, W., and Junker, A. 2017. 'Acutely damaged axons are remyelinated in multiple sclerosis and experimental models of demyelination', Glia, 65: 1350-60.

Schulze-Topphoff, U., Shetty, A., Varrin-Doyer, M., Molnarfi, N., Sagan, S. A., Sobel, R. A., Nelson, P. A., and Zamvil, S. S. 2012. 'Laquinimod, a quinoline-3-carboxamide, induces type II myeloid cells that modulate central nervous system autoimmunity', PLoS One, 7: e33797.

Shackleford, G., Sampathkumar, N. K., Hichor, M., Weill, L., Meffre, D., Juricek, L., Laurendeau, I., Chevallier, A., Ortonne, N., Larousserie, F., Herbin, M., Bieche, I., Coumoul, X., Beraneck, M., Baulieu, E. E., Charbonnier, F., Pasmant, E., and Massaad, C. 2018. 'Involvement of Aryl hydrocarbon receptor in myelination and in human nerve sheath tumorigenesis', Proc Natl Acad Sci U S A, 115: E1319-E28.

Shigetomi, E., Tong, X., Kwan, K. Y., Corey, D. P., and Khakh, B. S. 2011. 'TRPA1 channels regulate astrocyte resting calcium and inhibitory synapse efficacy through GAT-3', Nat Neurosci, 15: 70-80.

Shin, J. H., Zhang, L., Murillo-Sauca, O., Kim, J., Kohrt, H. E., Bui, J. D., and Sunwoo, J. B. 2013. 'Modulation of natural killer cell antitumor activity by the aryl hydrocarbon receptor', Proc Natl Acad Sci U S A, 110: 12391-6.

Silvestroff, L., Bartucci, S., Soto, E., Gallo, V., Pasquini, J., and Franco, P. 2010. 'Cuprizoneinduced demyelination in CNP::GFP transgenic mice', J Comp Neurol, 518: 2261-83.

Singh, K. P., Casado, F. L., Opanashuk, L. A., and Gasiewicz, T. A. 2009. 'The aryl hydrocarbon receptor has a normal function in the regulation of hematopoietic and other stem/progenitor cell populations', Biochem Pharmacol, 77: 577-87.

Skripuletz, T., Hackstette, D., Bauer, K., Gudi, V., Pul, R., Voss, E., Berger, K., Kipp, M., Baumgartner, W., and Stangel, M. 2013. 'Astrocytes regulate myelin clearance through recruitment of microglia during cuprizone-induced demyelination', Brain, 136: 147-67.

Skripuletz, T., Lindner, M., Kotsiari, A., Garde, N., Fokuhl, J., Linsmeier, F., Trebst, C., and Stangel, M. 2008. 'Cortical demyelination is prominent in the murine cuprizone model and is strain-dependent', Am J Pathol, 172: 1053-61.

Sofroniew, M. V. 2009. 'Molecular dissection of reactive astrogliosis and glial scar formation', Trends Neurosci, 32: 638-47.

Sofroniew, M. V., and Vinters, H. V. 2010. 'Astrocytes: biology and pathology', Acta Neuropathol, 119: 7-35.

Sofroniew, M.V. 2014. 'Astrogliosis', Cold Spring Harb Perspect Biol, 7: a020420.

Sospedra, M., and Martin, R. 2005. 'Immunology of multiple sclerosis', Annu Rev Immunol, 23: 683-747. 
Srinivasan, R., Lu, T. Y., Chai, H., Xu, J., Huang, B. S., Golshani, P., Coppola, G., and Khakh, B. S. 2016. 'New Transgenic Mouse Lines for Selectively Targeting Astrocytes and Studying Calcium Signals in Astrocyte Processes In Situ and In Vivo', Neuron, 92: 1181-95.

Stephenson, J. L., and Byers, M. R. 1995. 'GFAP immunoreactivity in trigeminal ganglion satellite cells after tooth injury in rats', Exp Neurol, 131: 11-22.

Stockinger, B., Hirota, K., Duarte, J., and Veldhoen, M. 2011. 'External influences on the immune system via activation of the aryl hydrocarbon receptor', Semin Immunol, 23: 99-105.

Stockinger, Brigitta, Meglio, Paola Di, Gialitakis, Manolis, and Duarte, João H. 2014. 'The Aryl Hydrocarbon Receptor: Multitasking in the Immune System', Annual Review of Immunology, 32: 403-32.

Sun, J., Shen, X., Dong, J., Zhao, J., Zuo, L., Wang, H., Li, Y., Zhu, W., Gong, J., and Li, J. 2015. 'Laquinimod ameliorates spontaneous colitis in interleukin-10-gene-deficient mice with improved barrier function', Int Immunopharmacol, 29: 423-32.

Suzuki, K. 1969. 'Giant hepatic mitochondria: production in mice fed with cuprizone', Science, 163: 81-2.

Taconic. 'Ahr Knockout Mouse, constitutive knockout', Accessed 11 April 2020. https://www.taconic.com/mouse-model/ahr-knockout-mouse\#.

Teva Pharmaceutical Industries Ltd. 2017. 'Teva and Active Biotech Announce CONCERTO trial of Laquinimod in RRMS Did Not Meet Primary Endpoint', Accessed 24 March 2020. http://ir.tevapharm.com/news-and-events/press-releases/press-releasedetails/2017/Teva-and-Active-Biotech-Announce-CONCERTO-trial-of-Laquinimod-inRRMS-Did-Not-Meet-Primary-Endpoint/default.aspx.

Thompson, A. J., Banwell, B. L., Barkhof, F., Carroll, W. M., Coetzee, T., Comi, G., Correale, J., Fazekas, F., Filippi, M., Freedman, M. S., Fujihara, K., Galetta, S. L., Hartung, H. P., Kappos, L., Lublin, F. D., Marrie, R. A., Miller, A. E., Miller, D. H., Montalban, X., Mowry, E. M., Sorensen, P. S., Tintore, M., Traboulsee, A. L., Trojano, M., Uitdehaag, B. M. J., Vukusic, S., Waubant, E., Weinshenker, B. G., Reingold, S. C., and Cohen, J. A. 2018. 'Diagnosis of multiple sclerosis: 2017 revisions of the McDonald criteria', Lancet Neurol, 17: 162-73.

Thöne, J., Ellrichmann, G., Seubert, S., Peruga, I., Lee, D. H., Conrad, R., Hayardeny, L., Comi, G., Wiese, S., Linker, R. A., and Gold, R. 2012. 'Modulation of autoimmune demyelination by laquinimod via induction of brain-derived neurotrophic factor', Am J Pathol, 180: 267-74.

Thöne, J., and Linker, R. A. 2016. 'Laquinimod in the treatment of multiple sclerosis: a review of the data so far', Drug Des Devel Ther, 10: 1111-8.

Toosy, A. T., Mason, D. F., and Miller, D. H. 2014. 'Optic neuritis', Lancet Neurol, 13: 83-99.

Torkildsen. 2008. 'The cuprizone model for demyelination'. 
Trapp, B. D., Peterson, J., Ransohoff, R. M., Rudick, R., Mork, S., and Bo, L. 1998. 'Axonal transection in the lesions of multiple sclerosis', N Engl J Med, 338: 278-85.

van Waesberghe, J. H., Kamphorst, W., De Groot, C. J., van Walderveen, M. A., Castelijns, J. A., Ravid, R., Lycklama a Nijeholt, G. J., van der Valk, P., Polman, C. H., Thompson, A. J., and Barkhof, F. 1999. 'Axonal loss in multiple sclerosis lesions: magnetic resonance imaging insights into substrates of disability', Ann Neurol, 46: 747-54.

Varrin-Doyer, M., Pekarek, K. L., Spencer, C. M., Bernard, C. C., Sobel, R. A., Cree, B. A., SchulzeTopphoff, U., and Zamvil, S. S. 2016. 'Treatment of spontaneous EAE by laquinimod reduces Th, B cell aggregates, and disease progression', Neurol Neuroimmunol Neuroinflamm, 3: e272.

Vasile, F., Dossi, E., and Rouach, N. 2017. 'Human astrocytes: structure and functions in the healthy brain', Brain Struct Funct, 222: 2017-29.

Vega-Riquer, J. M., Mendez-Victoriano, G., Morales-Luckie, R. A., and Gonzalez-Perez, O. 2019. 'Five Decades of Cuprizone, an Updated Model to Replicate Demyelinating Diseases', Curr Neuropharmacol, 17: 129-41.

Virchow, R. 1856. "Gesammelte Abhandlungen zyr Wissenschaftlichen Medizin." In. Frankfurt a.M.: Verlag von Meidinger Sohn \& Comp.

Voet, S., Prinz, M., and van Loo, G. 2019. 'Microglia in Central Nervous System Inflammation and Multiple Sclerosis Pathology', Trends Mol Med, 25: 112-23.

Vogel, C. F., Khan, E. M., Leung, P. S., Gershwin, M. E., Chang, W. L., Wu, D., HaarmannStemmann, T., Hoffmann, A., and Denison, M. S. 2014. 'Cross-talk between aryl hydrocarbon receptor and the inflammatory response: a role for nuclear factorkappaB', J Biol Chem, 289: 1866-75.

Vogel, C. F., and Matsumura, F. 2009. 'A new cross-talk between the aryl hydrocarbon receptor and RelB, a member of the NF-kappaB family', Biochem Pharmacol, 77: 734-45.

Vollmer, T. L., Sorensen, P. S., Selmaj, K., Zipp, F., Havrdova, E., Cohen, J. A., Sasson, N., GilgunSherki, Y., Arnold, D. L., and Group, Bravo Study. 2014. 'A randomized placebocontrolled phase III trial of oral laquinimod for multiple sclerosis', J Neurol, 261: 77383.

von Bartheld, C. S., Bahney, J., and Herculano-Houzel, S. 2016. 'The search for true numbers of neurons and glial cells in the human brain: A review of 150 years of cell counting', $J$ Comp Neurol, 524: 3865-95.

Waisman, A., Ginhoux, F., Greter, M., and Bruttger, J. 2015. 'Homeostasis of Microglia in the Adult Brain: Review of Novel Microglia Depletion Systems', Trends Immunol, 36: 62536.

Wake, H., Moorhouse, A. J., Jinno, S., Kohsaka, S., and Nabekura, J. 2009. 'Resting microglia directly monitor the functional state of synapses in vivo and determine the fate of ischemic terminals', J Neurosci, 29: 3974-80. 
Walisser. 2005. 'Aryl hydrocarbon receptor-dependent liver development and hepatotoxicity are mediated by different cell types'.

Wang, X. S., Cao, F., Zhang, Y., and Pan, H. F. 2020. 'Therapeutic potential of aryl hydrocarbon receptor in autoimmunity', Inflammopharmacology, 28: 63-81.

Warner, M., Mocarelli, P., Brambilla, P., Wesselink, A., Patterson, D. G., Jr., Turner, W. E., and Eskenazi, B. 2014. 'Serum TCDD and TEQ concentrations among Seveso women, 20 years after the explosion', J Expo Sci Environ Epidemiol, 24: 588-94.

Wegner, C., Stadelmann, C., Pfortner, R., Raymond, E., Feigelson, S., Alon, R., Timan, B., Hayardeny, L., and Brück, W. 2010. 'Laquinimod interferes with migratory capacity of T cells and reduces IL-17 levels, inflammatory demyelination and acute axonal damage in mice with experimental autoimmune encephalomyelitis', J Neuroimmunol, 227: 13343.

Weiner, H. L. 2008. 'A shift from adaptive to innate immunity: a potential mechanism of disease progression in multiple sclerosis', J Neurol, 255 Suppl 1: 3-11.

Wheeler, M. A., and Quintana, F. J. 2019. 'Regulation of Astrocyte Functions in Multiple Sclerosis', Cold Spring Harb Perspect Med, 9.

Williams, A., Piaton, G., and Lubetzki, C. 2007. 'Astrocytes--friends or foes in multiple sclerosis?', Glia, 55: 1300-12.

Williamson, M. A., Gasiewicz, T. A., and Opanashuk, L. A. 2005. 'Aryl hydrocarbon receptor expression and activity in cerebellar granule neuroblasts: implications for development and dioxin neurotoxicity', Toxicol Sci, 83: 340-8.

Winchenbach, J., Duking, T., Berghoff, S. A., Stumpf, S. K., Hulsmann, S., Nave, K. A., and Saher, G. 2016. 'Inducible targeting of CNS astrocytes in Aldh1l1-CreERT2 BAC transgenic mice', F1000Res, 5: 2934.

Wolf, Y., Shemer, A., Levy-Efrati, L., Gross, M., Kim, J. S., Engel, A., David, E., Chappell-Maor, L., Grozovski, J., Rotkopf, R., Biton, I., Eilam-Altstadter, R., and Jung, S. 2018. 'Microglial MHC class II is dispensable for experimental autoimmune encephalomyelitis and cuprizone-induced demyelination', Eur J Immunol, 48: 1308-18.

Yoon, H., Walters, G., Paulsen, A. R., and Scarisbrick, I. A. 2017. 'Astrocyte heterogeneity across the brain and spinal cord occurs developmentally, in adulthood and in response to demyelination', PLoS One, 12: e0180697.

Zhao, L., and Burt, A. D. 2007. 'The diffuse stellate cell system', J Mol Histol, 38: 53-64.

Zou, L. P., Abbas, N., Volkmann, I., Nennesmo, I., Levi, M., Wahren, B., Winblad, B., Hedlund, G., and Zhu, J. 2002. 'Suppression of experimental autoimmune neuritis by ABR215062 is associated with altered Th1/Th2 balance and inhibited migration of inflammatory cells into the peripheral nerve tissue', Neuropharmacology, 42: 731-9. 